\title{
Die Ballon-Okklusionsangiographie der Pulmonalarterien bei chronischer pulmonaler Hypertonie
}

\author{
Inauguraldissertation \\ zur Erlangung des Grades eines Doktors der Medizin \\ des Fachbereichs Medizin \\ der Justus-Liebig-Universität Gießen
}

vorgelegt von Andreas Breithecker

aus Merenberg

Gießen 2004 
Aus dem Medizinischen Zentrum für Radiologie Abteilung Diagnostische Radiologie

Leiter Prof. Dr. med. W.S. Rau

des Universitätsklinikums Gießen

Gutachter: Prof. Dr. W.S. Rau

Gutachter: Prof. Dr. Dr. Grimminger

Tag der Disputation: 13.01.2005 


\section{INHALTSVERZEICHNIS}

1. EINLEITUNG............................................................. 1

1.1 ANATOMIE DER LUNGENDURCHBLUTUNG.................. 1

1.2 PATHOPHYSIOLOGIE DER LUNGENDURCHBLUTUNG... 3

1.2.1 Kompensation einer beeinträchtigten pulmonalen

Zirkulation.................................................... 3

1.2.2 Chronische pulmonale Hypertonie........................ 3

1.3 BILDGEBUNG DER LUNGENPERFUSION...................... 6

1.3.1 Pulmonalisangiographie..................................... 6

1.3.2 Ventilations-Perfusionsszintigraphie..................... 7

1.3.3 Computertomographie............................ 8

1.3.4 Magnetresonanztomographie

1.3.5 Angiographische Befunde bei chronischer pulmonaler

Hypertonie.................................................... 9

1.3.6 Pulmonale Angioskopie......................................... 10

1.3.7 Pulmonalisangiographie bei chronischer pulmonaler

Hypertonie............................................................ 10

1.4 FRAGESTELLUNG DER VORLIEGENDEN ARBEIT............ 11

2. PATIENTEN, MATERIAL UND METHODEN............. 12

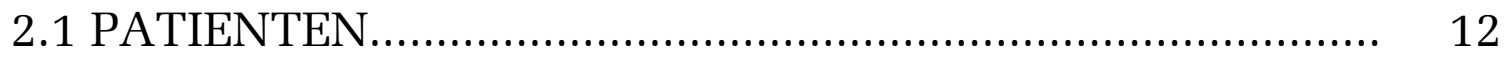

2.2 VORDIAGNOSTIK...................................................... 13

2.3 PULMONALISANGIOGRAPHIE....................................... 14

2.4 BALLON-OKKLUSIONSANGIOGRAPHIE.......................... 15

2.5 BEFUNDUNG .............................................................. 16

2.5.1 Übersichtsangiographie.......................................... 16 
2.5.2 Ballon-Okklusionsangiographie.................................. 19

2.6 STATISTISCHE AUSWERTUNG....................................... 23

2.6.1 Deskriptive univariate Methoden............................... 23

2.6.2 Inferenzstatistische univariate Methoden.................... 24

2.6.3 Deskriptive bivariate Methoden.................................. 25

2.6.4 Inferenzstatistische bivariate Methoden...................... 25

2.6.5 Prognosemodell..................................................... 26

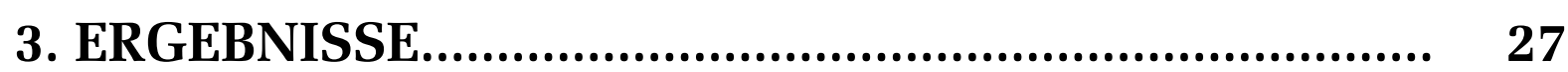

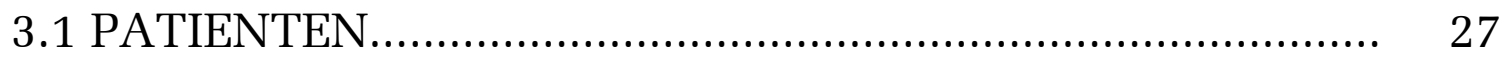

3.2 ANGIOGRAPHIE UND SZINTIGRAPHIE........................... 29

3.3 PARENCHYMMINDERPERFUSION................................. 31

3.4 THROMBEMBOLISCHE RESIDUEN............................... 35

3.5 METHODENVERGLEICH........................................... 39

3.6 METHODENVERGLEICH HINSICHTLICH

DER DIAGNOSE CTEPH............................................ 53

3.7 KOMPLIKATIONEN......................................................... 5

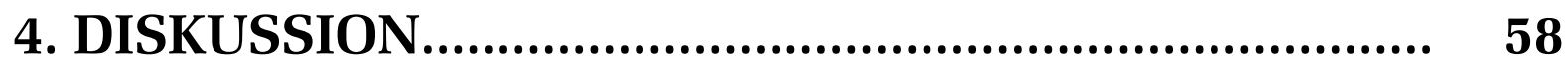

4.1 PATIENTENKOLLEKTIV .............................................. 59

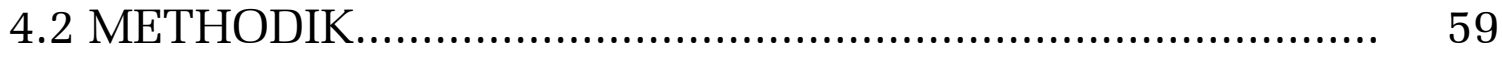

4.3 CHRONISCHE THROMBEMBOLISCHE

PULMONALE HYPERTONIE........................................ 60

4.3.1 Thrombembolische Residuen................................... $\quad 60$

4.3.2 Parenchymminderperfusion.................................... 68

4.3.3 Anastomosen und Kollateralen................................... 68

4.4 NICHT-THROMBEMBOLISCHE CHRONISCHE

PULMONALE HYPERTONIE......................................... 70 
5. ZUSAMMENFASSUNG

6. LITERATURVERZEICHNIS........................................ 77

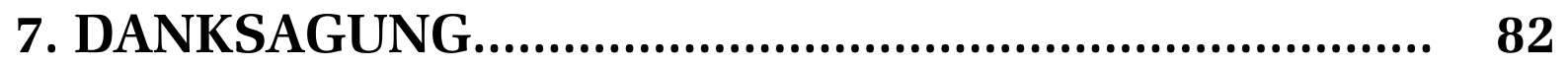

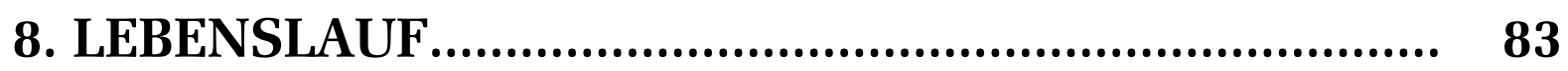


Die intraarterielle Pulmonalisangiographie ist der Goldstandard in der bildgebenden Diagnostik der Lungengefäße. Allerdings steht sie als invasives Untersuchungsverfahren am Ende der pulmonalen Stufendiagnostik. Bei der chronischen pulmonalen Hypertonie, die trotz verbesserter Therapiemöglichkeiten weiterhin als unheilbar gilt, ist sie Bestandteil der Ursachendiagnostik und wird dann durchgeführt, wenn Basisdiagnostik und Rechtsherzkatheteruntersuchung den Verdacht auf eine thrombembolische Genese erbringen oder wenn die Ätiologie der pulmonalen Drucksteigerung gänzlich unklar ist (Olschewski et al., 2002). Die Klärung dieser Fragen ist von Bedeutung, um den für den jeweiligen Patienten optimalen Therapieplan zu erstellen. Daher muss in der Diagnostik ein größtmögliches Maß an Genauigkeit angestrebt werden, denn die Pulmonalisangiographie liefert sowohl anatomische als auch funktionelle Informationen über das unmittelbare pathomorphologische Substrat der pulmonalen Hypertonie: die Pulmonalgefäße.

\subsection{Anatomie der Lungendurchblutung}

Die Durchblutung der Lunge erfolgt über zwei verschiedene Gefäßsysteme. Der Gasaustauschfunktion dienen die Vasa publica, das sind die Arteriae und Venae pulmonales. Sie bilden zusammen mit dem rechten Herzen den Lungenkreislauf. Die Pulmonalarterien folgen dem Verlauf der jeweiligen Bronchien in das Lungenparenchym hinein. Dabei besitzen sie bis etwa in die 7. Aufzweigungsgeneration einen elastischen Wandaufbau. Danach begleiten Arterien vom muskulären Bautyp die kleinen Bronchien. Daher erfolgt ihre Aufteilung auch genauso ungleichmäßig wie die des Bronchialbaumes. Duncker (1985) beschreibt diese irreguläre Dichotomie folgendermaßen: „Aus einem Bronchusstamm geht an der Aufzweigungsstelle annähernd in seiner Richtung ein stärkerer Ast hervor, der auch bis zu seiner nächsten Aufzweigung länger ist, während der dünnere und bis zur nächsten Aufzweigung kürzere Ast mit dem Stamm einen größeren Winkel bildet, also von der Richtung des Stammes deutlich abweicht. Verfolgt man den jeweils 
stärkeren Ast, so gelangt man in die vom Hilus weit entfernten Lungenabschnitte; diese stärkeren Äste zählen auch mehr Aufteilungsgenerationen als die jeweils schwächeren Äste, die die dem Hilus näher gelegenen Regionen versorgen. Dabei können sich die jeweils schwächeren Äste mit ihren größeren Abgangswinkeln auch über einige Teilungen bogenförmig ganz zurückwenden und die Regionen um die großen Bronchialstämme herum versorgen. Dieses Verhalten ist auf jeder Teilungsebene bis in die respiratorische Zone hinein zu beobachten“. Entlang der Bronchioli respiratorii findet man dann die Arteriolen mit einzelnen dünnen Muskelzellbündeln. Die Arteriolen gehen im weiteren Verlauf in das alveoläre Kapillarnetz über. Von dort aus sammelt sich nach erfolgtem Gasaustausch das sauerstoffreiche Blut zunächst in kleinen Venulen. Die sich daran anschließenden Lungenvenen verlaufen in den Interlobularsepten und den bindegewebigen Segmentgrenzen auf den Hilus zu. Nach Vereinigung zu insgesamt 4 Lungenvenen münden sie in den linken Vorhof (Duncker 1985).

Der Lungenkreislauf nimmt das gesamte Schlagvolumen des rechten Herzens auf. Demgegenüber transportieren die Rami oder Arteriae bronchiales als Vasa privata der Lunge lediglich ca. 1\% des Herzminutenvolumens (Frazier et al., 2000). Für die linke Lunge entspringen meist zwei Arterien direkt aus der thorakalen Aorta, während rechts in der Regel ein Hauptstamm aus der 3. oder 4. Interkostalarterie ebenfalls zwei Arterien hervorbringt. Die weiteren Aufzweigungen versorgen dann nach Abgabe von mediastinalen Seitästen die Bronchialwandstrukturen sowie die Wand der sie begleitenden Pulmonalarterien. Der venöse Abstrom des bronchialarteriellen Blutes erfolgt zentral über Vv. bronchiales in das Azygos- und Hemiazygossystem, weiter peripher direkt in die Vv. pulmonales (Duncker 1985).

Zwischen dem Lungenkreislauf und den Bronchialgefäßen als Teilen des Körperkreislaufes existieren mehrere Verbindungen. Die venöse Drainage der submukösen Venenplexus in den kleinen Bronchien erfolgt in das pulmonalvenöse System. Des Weiteren gibt es direkte Kurzschlüsse zwischen den Rami bronchiales und den Pulmonalarterienästen als sogenannte Rami pulmobronchiales (v. Hayek, 1970). Diese Sperrarterien besitzen einen kräftigen muskulären Wandaufbau und schaffen somit eine variable Verbindung zwischen Lungen- und Körperkreislauf. Weiter distal münden die aus Bronchialarterien hervorgehenden Kapillaren in die Vv. pulmonales, und auch im alveolären Kapillarnetz bestehen Verbindungen 
zwischen den Vasa privata und den Vasa publica. Schließlich sind auch in der Pleura visceralis Anastomosen zu finden.

\subsection{Pathophysiologie der Lungendurchblutung}

\subsubsection{Kompensation einer beeinträchtigten pulmonalen Zirkulation}

Die Bedeutung der bronchopulmonalen Verbindungen wird unter pathologischen Bedingungen der Lungenperfusion deutlich: Thrombembolien, Fremdkörperembolien oder aber Erkrankungen der Gefäßwand selbst mit Proliferation von Intima und Media führen zu einer Einengung oder völligen Verlegung des Gefäßlumens. Ist der Fluss in einer Pulmonalarterie dadurch reduziert, so ergeben sich Veränderungen im bronchialarteriellen Gefäßsystem. Die bronchiale Zirkulation weitet sich aus, hypertrophiert, proliferiert entlang der Anastomosen und steigert den Blutfluss um bis $\mathrm{zu}$ 300\% (Kauczor et al., 1994). Insbesondere im Falle einer zentralen Gefäßobstrukton, zum Beispiel durch eine Thrombembolie proximal der bronchopulmonalen Anastomosen, kommt es zu einem Kollateralfluss aus der systemischen Zirkulation in das pulmonalarterielle Gefäßbett distal des Verschlusses, wodurch die ischämische Nekrose des Lungenparenchymes in den meisten Fällen ausbleibt. Vielmehr kommt es nach Rekanalisation einer Lungenembolie meist wieder zu einer vollständigen Regeneration des abhängigen Lungenparenchymes.

\subsubsection{Chronische pulmonale Hypertonie}

Als Folge einer Lungenembolie kann sich jedoch auch eine chronische pulmonale Hypertonie entwickeln. Diese Diagnose wird dann gestellt, wenn der pulmonalarterielle Mitteldruck in Ruhe 25 und bei Belastung $30 \mathrm{mmHg}$ übersteigt. Zusätzlich liegt ein erhöhter pulmonaler Gefäßwiderstand vor. Über die Gesamtinzidenz der chronischen pulmonalen Hypertonie gibt es keine epidemiologischen Daten. Die primäre pulmonale Hypertonie als Prototyp der Erkrankung (Petkov et al., 2003) tritt mit einer jährlichen Inzidenz von einem bis zwei Fällen pro Million Einwohner auf (Abenhaim et al., 1996). Die Beschwerden der Patienten sind meist 
uncharakteristisch. Dies führt häufig dazu, dass die Diagnose verspätet oder gar nicht gestellt wird. Es finden sich eine verminderte Belastbarkeit, Belastungs- oder Ruhedyspnoe, Abgeschlagenheit, Angina, periphere Beinödeme und manchmal synkopale Ereignisse unter Belastung. Der Schweregrad der Erkrankung wird nach der WHO (1998) im Sinne einer modifizierten NYHA- Klassifikation und abhängig von der körperlichen Belastbarkeit in vier Klassen eingeteilt (Olschewski et al., 2002; Barst et al., 2004; Rich, 1998).

Unabhängig vom auslösenden pathogenetischen Mechanismus findet man in fortgeschrittenen Stadien ein uniformes pathohistologisches Bild an den kleinen präkapillären Lungengefäßen: Es kommt zu massiven Umbauvorgängen in der Gefäßwand mit Intimaproliferation, Mediahypertrophie und Ablagerungen von extrazellulärer Matrix in der Adventitia (Vascular Remodelling (Petkov et al., 2003)). Außerdem besteht eine Neigung zur Vasokonstriktion und zur Ausbildung von lokalen Thromben: in situ-Thrombose (Olschewski et al., 2002; Petkov et al., 2003). Die Veränderungen führen über eine Reduktion des Gefäßlumens zu einer Widerstandserhöhung. Die Folge ist eine Drucksteigerung im Lungenkreislauf mit zunehmender Rechtsherzbelastung und Ausbildung eines Cor pulmonale mit seinen Komplikationen.

Die neueste Klassifikation der WHO aus dem Jahre 1998 (Evian/Frankreich) unterscheidet fünf Gruppen der chronischen pulmonalen Hypertonie:

In Gruppe 1 findet man die Erkrankungsformen, bei denen die Auslöser der pulmonalen Drucksteigerung nicht bekannt sind, zum Beispiel die primäre pulmonale Hypertonie (PPH). Als Behandlungsmöglichkeiten kommen neben der Langzeittherapie mit Sauerstoff die symptomatische Senkung des pulmonalarteriellen Druckes (z.B. mit Kalziumantagonisten) und die Beeinflussung der Umbauvorgänge in den Gefäßwänden (beispielsweise mit Prostazyklinen, Endothelinrezeptorantagonisten oder Sildenafil) zum Einsatz.

In der Gruppe 2 sind die Erkrankungen zusammengefasst, die über eine pulmonalvenöse Abflussbehinderung durch Rückstau eine pulmonalarterielle Drucksteigerung bewirken. Am häufigsten sind in dieser Gruppe die kongestiven Herzerkrankungen. Bei der pulmonalen venookklusiven Erkrankung handelt es sich um eine sehr seltene idiopathische Form der pulmonalvenösen Hypertonie. Pathoanatomisch finden sich netzartige Septen (Webs), rekanalisierte Thrombosen und eine Fibrosierung der Intima in den Lungenvenen (Frazier et al., 2000). Die Folge 
ist eine in verschiedenen Lokalisationen variabel ausgeprägte Obliteration des Venenlumens mit Erhöhung des kapillären Verschlussdruckes und schließlich eine sekundäre pulmonalarterielle Hypertonie.

Gruppe 3 enthält Erkrankungen, die zu einer alveolären Hypoxie führen und durch die Eisenmenger-Reaktion zunächst eine reversible, im langfristigen Verlauf dann aber eine bleibende pulmonale Hypertonie erzeugen.

In Gruppe 4 werden Erkrankungen aufgeführt, bei denen eine lokale Thrombenbildung oder Embolien die initiale Drucksteigerung durch Reduktion des Gesamtgefäßquerschnittes auslösen. Die häufigste Erkrankung aus dieser Gruppe ist die chronische thrombembolische pulmonale Hypertonie (CTEPH). Genaue Angaben über Inzidenz und Prävalenz der CTEPH existieren nicht. Man nimmt jedoch an, dass sie etwa um ein Drittel häufiger ist als die primäre pulmonale Hypertonie (PPH) (Kunieda et al., 1999). In den USA schätzt man die Prävalenz der chronischen pulmonalen Hypertonie auf 0,1 bis 0,5\% unter den Patienten, die eine Lungenembolie überlebt haben (Moser et al., 1990; Jamieson und Kapelanski, 2000). Zwei wichtige Faktoren führen dazu, dass sich aus einer einmaligen oder immer wieder rezidivierenden Lungenembolien eine bleibende Gefäßobliteration entwickelt: Einerseits kann die Lungenembolie nahezu asymptomatisch oder aber atypisch verlaufen, so dass keine oder nur eine inadäquate Behandlung erfolgt. Andererseits kommt es bei 0,4 bis 5\% der Patienten nicht zu einer vollständigen Thrombolyse (Kauczor et al., 2000). Dann wird das thrombembolische Material in die Gefäßwand eingebaut mit der Folge einer bleibenden Reduktion des Gesamtquerschnittes der Pulmonalisstrombahn. Beginnen diese thrombembolischen Veränderungen der Gefäßwand auf Höhe der Segmentarterien oder proximal davon, steht mit der pulmonalen Thrombendarteriektomie ein chirurgisches Verfahren zur Verfügung, das eine wirksame kausale Behandlung der Erkrankung mit guten Langzeitergebnissen erlaubt (Mayer et al., 1996; Moser et al., 1987; Archibald et al., 1999). Dabei wird der präoperativen Diagnostik abverlangt, dass sie eindeutige Aussagen über die Operabilität und die Lokalisation der Befunde liefert. Außerdem muss eine Vorhersage gemacht werden, ob der Patient von dieser chirurgischen Maßnahme profitiert.

In der Gruppe 5 sind die Grunderkrankungen einer pulmonalen Hypertonie aufgeführt, die sich unmittelbar vor Ort am Gefäß selbst abspielen. Dabei wird noch diskutiert, ob die primäre kapilläre Hämangiomatose $(\mathrm{PCH})$ als eigenständige 
Entität aufzufassen ist, oder ob es sich um eine mögliche Ausprägungsform der pulmonalen venookklusiven Erkrankung (PVOD) handelt (Frazier et al., 2000).

\subsection{Bildgebung der Lungenperfusion}

\subsubsection{Pulmonalisangiographie}

Die Pulmonalisangiographie in Blattfilm-Technik ist das älteste Verfahren zur direkten Bildgebung der Pulmonalarterien. Erste Publikationen reichen in die späten Dreißigerjahre zurück (Robb und Steinberg, 1938). Bei ihrer Einführung wurde Röntgenkontrastmittel intravenös oder in den rechten Vorhof injiziert. Die selektive Pulmonalisangiographie wurde erstmals 1969 beschrieben (Bookstein, 1969). Dabei wird über einen in einem Pulmonalarterienhauptstamm, einer Lappen- oder sogar Segmentarterie platzierten angiographischen Katheter jodhaltiges Röntgenkontrastmittel injiziert. Während der Injektion wird automatisch eine Serie von konventionellen, analogen Röntgenbildern der Lunge erstellt.

Eine Weiterentwicklung der Methode konnte in den Achtzigerjahren durch die Einführung der Digitalen Subtraktionsangiographie (DSA) erzielt werden. Hierbei fertigt man eine digitale Bildserie an. Nach einer variablen Anzahl von Leerbildern wird die Injektion des Kontrastmittels ausgelöst. Durch die rechnergestützte Subtraktion einer vor Kontrastmittelgabe angefertigten Maske vom eigentlichen Füllungsbild werden störende knöcherne Strukturen eliminiert, und man erhält ein überlagerungfreies Bild des kontrastierten Gefäßbaumes. In mehreren Studien konnte gezeigt werden, dass die digitale Angiographie in der Diagnostik der akuten Lungenembolie der Blattfilm-Technik mindestens gleichwertig ist (Smith et al., 2002; Johnson et al., 1998; Hagspiel et al., 1998; van Beek et al., 1996). Materialund Zeitaufwand waren jedoch geringer. Die Methode ist allerdings störanfällig gegenüber Bewegungsartefakten zum Beispiel bei dyspnoeischen Patienten. Ferner ist die intraarterielle Pulmonalisangiographie, sei es in Blattfilm- oder in DSATechnik, ein invasives Untersuchungsverfahren mit entsprechendem Risikopotential.

Die Ballon-Okklusionsangiographie der Pulmonalarterien ist in der Literatur erstmals 1963 beschrieben (Jacobson). In verschiedenen Studien wurde ihr Nutzen in 
der Diagnostik der akuten Lungenembolie, des ARDS und von Gefäßveränderungen im Rahmen angeborener oder erworbener Herzerkrankungen evaluiert (Wilson und Bynum, 1976; Bynum und Wilson, 1979; Ferris et al., 1984; Greene et al., 1983; Wilson et al., 1993).

Mit einem Katheter, der an seiner Spitze einen inflatierbaren Ballon trägt, wird eine Lappen-, Segment- oder Subsegmentarterie selektiv sondiert. Dann wird der Ballon entfaltet, um das Gefäß vorübergehend zu verschließen. Nach manueller Kontrastmittelinjektion werden die gefüllten Gefäßverzweigungen detailliert dargestellt. Die Bilddokumentation in vorangegangenen Arbeiten erfolgte meist als Cineangiographie auf Videoband oder als Spotfilm-Angiographie in hochaufgelösten digitalen Radiographien (Wilson und Bynum, 1976; Bynum und Wilson, 1979).

\subsubsection{Ventilations-Perfusionsszintigraphie}

Auch die Szintigraphie hat sich in der Diagnostik der Lungenembolie etabliert. Als nichtinvasives Screeningverfahren kommt sie zum Nachweis einer Lungenembolie vor allem dort zum Einsatz, wo aufgrund der Geräteausstattung vor Ort weder Computertomographie noch Pulmonalisangiographie im Akutfall zu Verfügung stehen. Außerdem hat sie sich beim Nachweis peripherer Embolien als komplementäres Verfahren zur Spiral-CT, die Embolien nur bis in das Niveau der Subsegmentarterien zuverlässig nachweist, bewährt.

Bei der chronischen pulmonalen Hyperonie spielt sie eine entscheidende Rolle beim Nachweis einer thrombembolischen Genese. Dann zeigt sie nämlich einen oder mehrere, diskordante mindestens segmentale Perfusionsdefekte ohne begleitende Belüftungsstörung. Im Gegensatz dazu findet man bei anderen Formen der pulmonalen Hypertonie eine normale Perfusion oder subsegmentale, „mottenfraßartige“ Defekte (Fedullo et al., 2001). Allerdings unterschätzt die Szintigraphie häufig das Ausmaß der pulmonalen Gefäßobstruktion im Vergleich zur Angiographie oder zum intraoperativen Aspekt (Ryan et al., 1988). 


\subsubsection{Computertomographie}

Die Computertomographie berechnet transversale Querschnittsbilder anhand einer computergesteuerten quantitativen Messung der Schwächung von Röntgenstrahlen beim Durchtritt durch den abgebildeten Körperabschnitt (Lorenz R, 1991).

In den Neunzigerjahren ist mit der Spiral-Computertomographie das erste Schnittbildverfahren in der Diagnostik der Lungenembolie hinzugekommen. Entscheidend hierfür war die deutliche Beschleunigung der Untersuchungsgeschwindigkeit gegenüber der ursprünglichen Technik. Dadurch konnte der gesamte pulmonale Gefäßbaum nach bolusartiger Kontrastmittelinjektion in der optimalen Kontrastierungsphase erfasst werden. Gleichzeitig wurde die räumliche Auflösung durch kontinuierlich verbesserte Detektortechnologie immer weiter gesteigert. Dieser Trend setzte sich auch dann noch fort, als mit der Mehrzeilen-Spiral-CT eine neue Gerätegeneration aufkam. Durch diese wichtige Neuerung etablierte sich die Angio-Computertomographie (Angio-CT, CT-Angiographie) der Pulmonalarterien als Maßnahme der ersten Wahl, um akute Lungenembolien abzubilden. Der Thrombembolus ist dabei direkt und zuverlässig bis in das Niveau der Subsegmentarterien nachweisbar (Schoepf und Costello, 2004). Aber auch die Residuen abgelaufener Embolien in Form von Wandauflagerungen, Füllungsdefekten, sowie Verschlüssen werden mit vergleichbarer Sensitivität erfasst (Pitton et al., 2002). Der Gefäßbaum kann als 3D-Rekonstruktion in einer aus der konventionellen Angiographie bekannten Weise anschaulich dargestellt werden. Allerdings ermöglicht der dreidimensionale nahezu isotrope Datensatz die Betrachtung der Gefäßmorphologie aus beliebigen Raumrichtungen. Obendrein erlaubt die Computertomographie die gleichzeitige Erfassung des Lungenparenchymes und der mediastinalen Organe. Einerseits hilft dies in der Differentialdiagnose des akuten Thoraxschmerzes (z.B. Aortendissektion, Pneumothorax, Pneumonie). Andererseits werden Lungenparenchymerkrankungen, die eine chronische pulmonale Hypertonie verursachen (z.B. Lungenfibrose, Emphysem) zuverlässig miterfasst. 


\subsubsection{Magnetresonanztomographie}

Die Magnetresonanztomographie (MRT, NMR) erzeugt Schnittbilder in beliebigen Ebenen. Die Emission elektromagnetischer Energie im statischen Magnetfeld nach Anregung durch einen Hochfrequenzimpuls wird als Signal gemessen. Die unterschiedliche Signalintensität der verschiedenen Gewebe wird im Graustufenbild dargestellt (Lorenz R, 1991). Das Signalverhalten kann dabei durch paramagnetische Substanzen im Sinne einer Kontrastverstärkung beeinflusst werden. Auch die durch MRT erstellten Schnittbilder eignen sich für eine dreidimensionale Rekonstruktion. Die Magnetresonanztomographie als kontrastmittelverstärkte MRAngiographie kann bis in Höhe der Segmentarterien sicher Lungenembolien nachweisen. Dabei sind Sensitivität und Spezifität mit der CT (Bongartz et al., 1998) vergleichbar. Im Tiermodell fanden Seo et al. 2003 sogar eine höhere Sensitivität der MR-Angiographie im Nachweis peripherer Embolien.

Allerdings erfordert die Überwachung der oft schwer kranken Patienten einen deutlich größeren logistischen Aufwand im Vergleich zur Computertomographie, da der Patient im Tomographen kaum zugänglich ist und nur spezielle Materialien und Überwachungsgeräte eingesetzt werden können.

Insbesondere als Verlaufskontrolle unter laufender Medikation oder postoperativ wird sie eine zunehmend wichtige Bedeutung erhalten. Sie kann nämlich in einem Untersuchungsgang nichtinvasiv sowohl die Gefäßmorphologie abbilden als auch mit flusssensitiven Sequenzen eine Abschätzung der pulmonalarteriellen Drucke im Verlauf ermöglichen (Ley et al., 2003). Hinzu kommen die bereits etablierten Methoden der Kardio-MRT.

\subsubsection{Angiographische Befunde bei chronischer pulmonaler Hypertonie}

Typische angiographische Befunde der primären pulmonalen Hypertonie sind die symmetrische Aufweitung der zentralen Pulmonalarterien, Kalibersprünge sowie Gefäßabbrüche subsegmental und weiter peripher. Außerdem präsentiert sich das Bild fadenförmiger oder korkenzieherartig gewundener Gefäße in der Peripherie. Subpleural sind manchmal Kollateralen abgebildet. Bei der CTEPH demonstriert die Pulmonalisangiographie die organisierten Restthromben in Form von Füllungs- 
defekten, die als Wandauflagerungen oder Stenosen imponieren können. Membranartige fadenförmige Restthromben werden als Strickleitersysteme oder Webs und Bands bezeichnet. Ferner fallen fehlende arterielle Segmente und abrupte Gefäßabbrüche auf. In den Parenchymbezirken distal einer Embolie ist die Kontrastanfärbung reduziert oder aufgehoben.

Bei der PVOD wurden eine Dilatation der zentralen Pulmonalarterien und des rechten Ventrikels, eine verlängerte Parenchymanfärbung sowie eine verzögerte Venenfüllung beobachtet (Shackelford et al., 1977; Matsumoto et al., 1993).

\subsubsection{Pulmonale Angioskopie}

Die Angioskopie der Pulmonalarterien wurde speziell für die präoperative Evaluation entwickelt. Bei der CTEPH zeigt sie Vernarbungen und Rauhigkeiten der intimalen Gefäßoberfläche, Webs und Bands, die das Gefäßlumen durchqueren, und organisierte Thromben.

Die Angioskopie wird durchgeführt, wenn bei Patienten mit milder pulmonaler Hypertonie und in der Angiographie nicht abgrenzbarer proximaler Ausdehnung der thrombembolischen Residuen der hämodynamische Effekt einer möglichen Thrombendarteriektomie vorhergesagt werden soll. Außerdem kommt sie zum Einsatz bei Patienten mit CTEPH und sehr schwerer pulmonaler Druckerhöhung, bei denen der Angiographiebefund nur peripher gelegene Veränderungen zeigt (Fedullo et al., 2001).

\subsubsection{Pulmonalisangiographie bei chronischer pulmonaler Hypertonie}

Die rasante Entwicklung der angiographischen Schnittbildverfahren hatte zur Folge, dass die intraarterielle Pulmonalisangiographie als invasive und somit potentiell komplikationsbehaftete Methode im diagnostischen Algorithmus der Lungenembolie weit zurückgedrängt wurde. Als Goldstandard kommt sie bei der Frage nach akuter Lungenembolie nur noch in Zweifelsfällen zur Anwendung.

Bei Patienten mit chronischer pulmonaler Hypertonie ist sie jedoch als Referenzmethode ergänzend zu CT und Szintigraphie erforderlich, wenn die Operabilität 
der thrombembolischen Form geklärt werden soll, oder wenn bei ätiologischer Unklarheit periphere Embolieresiduen gesucht werden. Insbesondere zum besseren Nachweis von thrombembolischen Residuen in den kleinen Pulmonalarterien erscheinen dabei ergänzende spezielle Techniken erforderlich wie die Ballon-Okklusions-Angiographie, die superselektive Angiographie oder die Cineangiographie (Stein et al., 1999). Es konnte nämlich gezeigt werden, dass gerade Lungenembolien in subsegmentalen Arterien und weiter peripher mit der alleinigen Übersichtsangiographie unzuverlässig detektiert werden mit einer reduzierten Untersucher-Übereinstimmung (Inter-Observer-Aggreement).

\subsection{Fragestellung der vorliegenden Arbeit}

Ziel der vorliegenden retrospektiven Analyse ist es zu untersuchen, inwieweit die zusätzlich zur konventionellen Pulmonalisangiographie angefertigte selektive Ballon-Okklusions-Angiographie die diagnostische Aussagekraft bei Patienten mit chronischer pulmonaler Hypertonie erhöht. Wilson und Bynum berichten 1976 über die Vorteile der Technik im Nachweis von akuten Lungenembolien: Die größeren Gefäße können besser definiert werden. Aufgrund der selektiven Katheterlage und des fehlenden Auswascheffektes durch Einstrom nichtkontrastierten Blutes unter Okklusionsbedingungen sind Gefäßabbrüche und Füllungsdefekte eindeutiger zu identifizieren. Die kleineren Gefäße mehrere Generationen distal der Segmentarterien sind besser sichtbar. Dies verbessert auch die Nachweisbarkeit kleiner, mit anderen Techniken nicht auffindbarer Embolien. Gerade in den basalen Lungenanteilen kann vermieden werden, dass Gefäße aufgrund von Überlappungen mit benachbarten Gefäßen nicht beurteilt werden können.

Weiterhin galt es in dieser Arbeit zu überprüfen, ob auch pathophysiologische Kompensationsmechanismen - wie zum Beispiel die Hypertrophie der Bronchialarterien - im angiographischen Bild festgehalten werden können, und ob sich

hieraus Prognosen über den Effekt einer geplanten Revaskularisation durch pulmonale Thrombendarteriektomie ableiten lassen. 


\subsection{Patienten}

Zum Vergleich der konventionellen Darstellung der Pulmonalgefäße mittels Übersichtsangiographie in zwei Ebenen und der Ballon-Okklusionsangiographie wurden Daten von insgesamt 138 Patienten im Zeitraum von Dezember 1999 bis Mai 2004 herangezogen. Dabei handelte es sich um 67 Frauen und 71 Männer im Alter von 18 bis 75 Jahren. Das Spektrum der Diagnosen ist in Tabelle 1.1 abzulesen.

\begin{tabular}{l|r}
\hline Diagnose & Patienten \\
\hline CTEPH & 94 \\
No-CTEPH & 7 \\
CTEPH+PPH & 1 \\
CTEPH+COPD & 1 \\
DD CTEPH/PPH & 1 \\
PPH & 13 \\
Kollagenose & 4 \\
Fibrose & 2 \\
EAA, EAA/PVOD & 3 \\
PVOD & 2 \\
PCH & 1 \\
COPD & 2 \\
Kardial & 2 \\
Portopulmonal & 1 \\
Sonstige & 2 \\
unklar & 2 \\
\hline
\end{tabular}

Tab. 1.1: Diagnosenspektrum des Gesamtkollektives

Primär interessierte uns, ob die Ballon-Okklusionsangiographie bessere, d.h. detailliertere Untersuchungsergebnisse liefert. Zu diesem Zweck wurden aus dem oben beschriebenen Gesamtkollektiv die Untersuchungen von 50 konsekutiven Patienten (März 2001 bis Januar 2003), 24 Frauen und 26 Männer im Alter von 27 bis 74 Jahren, nach einem vorgefertigten Studienprotokoll (vgl. 2.5) retrospektiv ausgewertet. Einschlusskriterium war das Vorliegen einer chronischen pulmonalen Hypertonie. Das Diagnosenspektrum dieser Patientengruppe ergibt sich aus Tabelle 1.2. 


\begin{tabular}{l|r}
\hline Diagnose & Patienten \\
\hline CTEPH & 37 \\
PPH & 5 \\
Kollagenose & 2 \\
COPD & 2 \\
PCH & 1 \\
Aortenstenose & 1 \\
DD & \\
EAA/PVOD & 1 \\
unklar & 1 \\
\hline
\end{tabular}

Tab. 1.2: Diagnosenspektrum des Studienkollektives

Die Ausführungen über die verwendeten Untersuchungs- und Auswertungsmethoden in den folgenden Abschnitten beziehen sich auf dieses Studienkollektiv (vgl. 2.2 bis 2.6).

Die Daten aus dem eingangs beschriebenen Gesamtkollektiv dienten darüber hinaus der Beschreibung von sporadischen Beobachtungen bei geringer Fallzahl (z.B. das angiographische Erscheinungsbild bei Patienten mit pulmonaler venookklusiver Erkrankung (PVOD) oder primärer kapillärer Hämangiomatose (PCH)). Außerdem wurden die 88 Patienten aus dem Gesamtkollektiv, die nicht zum Studienkollektiv gehörten, zu einer Kontrollgruppe zusammengefasst. Diese wurde unter Verwendung eines Wahrscheinlichkeitsmodells für die Schätzung der Auftrittswahrscheinlichkeit von thrombembolischen Residuen in der BallonOkklusionsangiographie bei negativer Übersichtsangiographie herangezogen (vgl. 2.6).

\section{$2.2 \quad$ Vordiagnostik}

Bei allen Patienten wurden als nichtinvasive bildgebende Diagnostik eine Ventilations-Perfusions-Szintigraphie und eine Echokardiographie durchgeführt. Außerdem wurden eine Angio-Computertomographie (CT-Angiographie) der Pulmonalarterien und eine hochauflösende Computertomographie (High-resolution CT, HR-CT) des Lungenparenchymes angefertigt.

In zeitlicher Nähe zur Pulmonalisangiographie erfolgte eine Rechtsherzkatheteruntersuchung mit Bestimmung der pulmonalen Druck- und Widerstandsver- 
hältnisse sowie pharmakologischer Testung unter anderem auf Ansprache nach Sauerstoff-, Stickstoffmonoxid- oder Iloprost-Gabe (stabiles Prostazyklinanalogon).

\subsection{Pulmonalisangiographie}

Die Indikation zur intraarteriellen Pulmonalisangiographie wurde in 29 Fällen zur Klärung der Operabilität bei bereits bekannter Diagnose CTEPH gestellt. 21-mal wurde sie zur Diagnosefindung bei unklarer Ätiologie der chronischen pulmonalen Hypertonie veranlasst.

Nach Aufklärung und Einwilligung des Patienten wurde ein geeigneter Zugangsweg für die Katheterisierung festgelegt. In allen Fällen erfolgte die Untersuchung über die V. femoralis communis (41-mal rechts, 9-mal links). Insbesondere während des erforderlichen Katheterwechsels im Rahmen der folgenden Ballon-Okklusionsangiographie stellte sich die Femoralvene als günstigstes Zugangsgefäß heraus. Der Patient wurde flach auf dem Untersuchungstisch gelagert. Allen Patienten wurde kontinuierlich Sauerstoff über eine Nasensonde in einer Dosis von zwei bis sechs Litern pro Minute verabreicht. Nach Hautdesinfektion und sterilem Abdecken erfolgte in üblicher Technik die Lokalanästhesie mit 10 bis $20 \mathrm{ml}$ Prilocainhydrochlorid 1\% (Xylonest 1\% ${ }^{\circledR}$ ). Anschließend wurde das Zugangsgefäß mit einer Hohlnadel punktiert. In Seldinger-Technik erfolgte die Einlage einer 7F-Schleuse (Terumo Europe). Mittels Führungsdraht (Stiff Type, Terumo Europe) und ausreichend langem Angiographiekatheter (Pigtail 7F 110 cm, Cordis/JohnsonJohnson) wurde zunächst unter Durchleuchtungskontrolle die rechte Pulmonalarterie selektiv sondiert. Die rechte Lunge wurde in zwei Ebenen untersucht, zunächst in RAO- (mit angehobenem rechtem Oberkörper) und anschließend in seitlicher Projektion. Pro Untersuchungsserie wurden $45 \mathrm{ml}$ verdünntes (Kochsalzlösung) Kontrastmittel (Ultravist, Schering/Berlin, $300 \mathrm{mg} \mathrm{J} / \mathrm{ml}$ ) mit einer Geschwindigkeit von $25 \mathrm{ml} / \mathrm{s}$ maschinell injiziert.

Die Angiographie in DSA-Technik erfolgte an einer Mehrzweckanlage mit Übertischröhre und einem $40 \mathrm{~cm}$ großen Bildverstärkersystem mit einer Frequenz von drei Bildern pro Sekunde. Die digitale Bildmatrix betrug 1024x1024 Pixel (Fluorospot TOP, Siemens Erlangen, Abb. 1). 


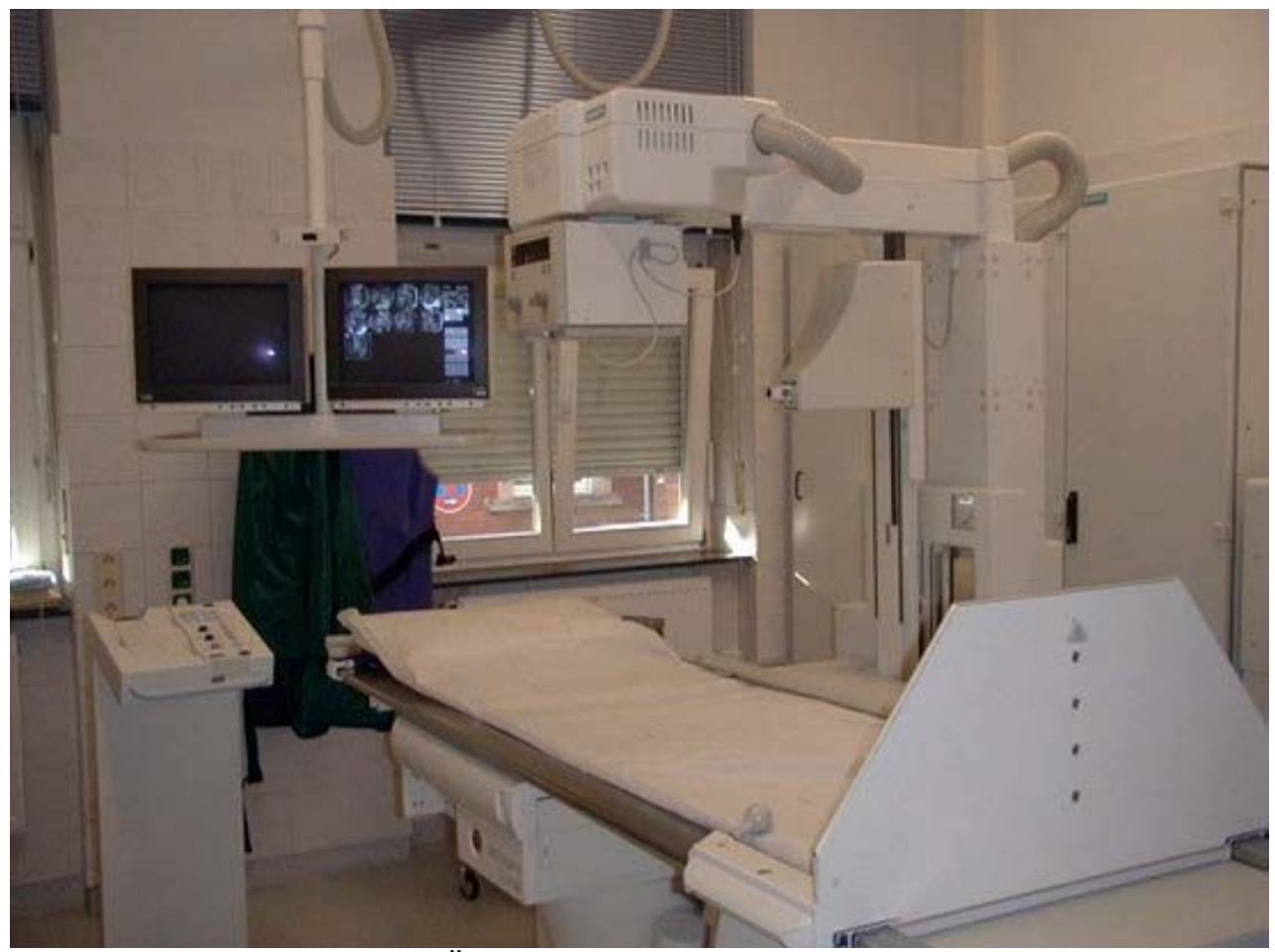

Abb. 1: Angiographiegerät mit Übertischröhre (Siemens Fluorospot TOP)

Meist war es möglich, die Aufnahmeserien in Atemstillstand anzufertigen, bei dyspnoeischen Patienten erwies sich jedoch eine kontinuierliche flache Atmung als günstiger.

Danach erfolgte die Sondierung der linken Pulmonalarterie und die linke Lunge wurde in gleichartiger Weise in zwei Ebenen (LAO und seitlich) untersucht.

Die Bildnachbearbeitung erfolgte an der Konsole des Angiographiegerätes: Auswahl einer geeigneten Maske zur Optimierung des Subtraktionseffektes, Justierung von Kontrast und Helligkeit, Speicherung von Bildern in der arteriellen Füllungsphase, in der kapillären oder Parenchymphase und in der Phase des venösen Abstromes. Diese Bilder wurden sowohl auf Film als auch digital dokumentiert.

\subsection{Ballon-Okklusionsangiographie}

Nachdem beide Lungen selektiv in zwei Ebenen in der Übersichtsangiographie dargestellt wurden, erfolgte über den Führungsdraht der Austausch des einliegenden Pigtail-Katheters gegen einen weichen, auf maximal 26 French dehnbaren Latex-Ballonkatheter (Standard Occlusion Balloon Catheter, Boston Scientific/MEDI-TECH). Es wurden in beiden Lungen repräsentativ jeweils zwei bis drei Gefäße auf Segment- oder Subsegmentniveau unter Durchleuchtungskontrolle 
selektiv sondiert. Durch kurze manuelle Kontrastmittelinjektion konnte man dabei das Kaliber des sondierten Gefäßes überprüfen. Bei korrekter Katheterlage wurde dann der weiche Latexballon des Katheters über den Seitport mit einem Gemisch aus Kochsalzlösung und Kontrastmittel unter kontinuierlicher Durchleuchtungskontrolle vorsichtig inflatiert. Der Vorgang wurde dann beendet, wenn der Ballon den Durchmesser des Gefäßes erreicht hatte. Dies ist visuell daran zu erkennen, dass sich der zunächst kreisrunde Ballon an den Seiten abflacht. Das sondierte Gefäß war nun okkludiert.

Der Führungsdraht wurde entfernt, und der Arbeitskanal des Katheters musste mit einem Gemisch aus Kochsalzlösung und niedrig dosiertem Heparin (200 IE/100 ml) ausreichend gespült werden $(20 \mathrm{ml})$. Für die nun folgende Angiographieserie wurde das Kontrastmittel ebenfalls auf $300 \mathrm{mg} \mathrm{J} / \mathrm{ml}$ verdünnt. Das Injektionsvolumen betrug pro Serie etwa 10 bis $15 \mathrm{ml}$ und wurde per manueller Injektion verabreicht. Bei maximaler Vergrößerung, an unserem Gerät einem Bildverstärker von $14 \mathrm{~cm}$ entsprechend, wurden DSA-Serien mit 2 Bildern/s (vgl. 2.3) angefertigt. Die Bildnachbearbeitung und -dokumentation entsprach dem bereits im vorangegangenen Kapitel beschriebenen Vorgehen (vgl. 2.3).

\subsection{Befundung}

Die nachbearbeiteten und anonymisierten Bilder wurden von zwei angiographisch erfahrenen Radiologen primär im Konsens ausgewertet. Dabei wurden die Untersuchungen nach einem vorgefertigten Protokoll systematisch analysiert:

Für jeden Patienten wurden zahlreiche Merkmale registriert, mittels derer die Methoden beurteilt wurden. Die Bildbetrachtung und die manchmal nochmals erforderliche Nachbearbeitung (Optimierung von Kontrast und Helligkeit etc.) erfolgten sowohl an einer Befundungsworkstation (RADWORKS) als auch ergänzend mit kommerziell erhältlicher Bildbearbeitungssoftware (Corel PhotoPaint).

\subsection{1 Übersichtsangiographie}

Im ersten Auswertungsschritt wurde die Übersichtsangiographie in zwei Ebenen befundet. Zunächst erfolgte die Abschätzung der allgemeinen Beurteilbarkeit der zwei Hauptarterien und der fünf Lappenarterien. Die Beurteilbarkeit der distal der 
Lappenarterien gelegenen Teilungsgenerationen wurde dann exemplarisch im rechten Oberlappen überprüft. Dabei war ein Gefäßabschnitt auszuwählen, der nicht von proximalen Verschlüssen betroffen war. Der rechte Oberlappen eignet sich am besten zu diesem Zweck, weil es hier am wenigsten zu Überlagerungen von Gefäßen in ventrodorsaler Richtung kommt und somit das Auszählen der Gefäßverzweigungen zuverlässig gelingt (Abb.2).

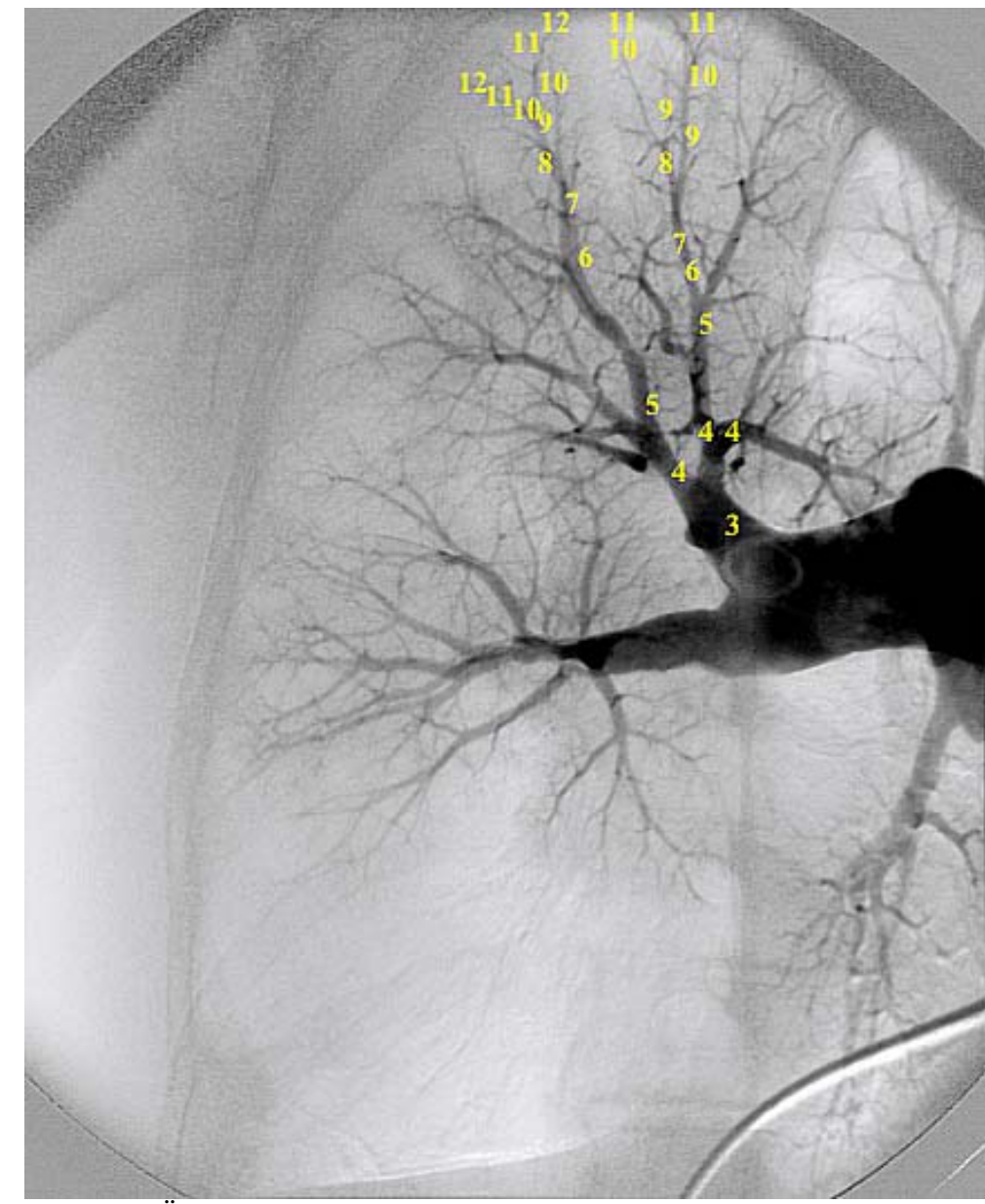

Abb. 2: Übersichtsangiographie der rechten Lunge. Auszählungsweise der Aufzweigungsgenerationen im rechten Oberlappen. Außerdem ist ein Verschluss der rechten Unterlappenarterie zu erkennen.

Ferner wurden die selektiv in der Okklusionsangiographie dargestellten Gefäßpfade mit der Übersichtsangiographie sowohl bezüglich der Beurteilbarkeit als auch der abgebildeten Gefäße verglichen (Abb. 3, S. 18). 

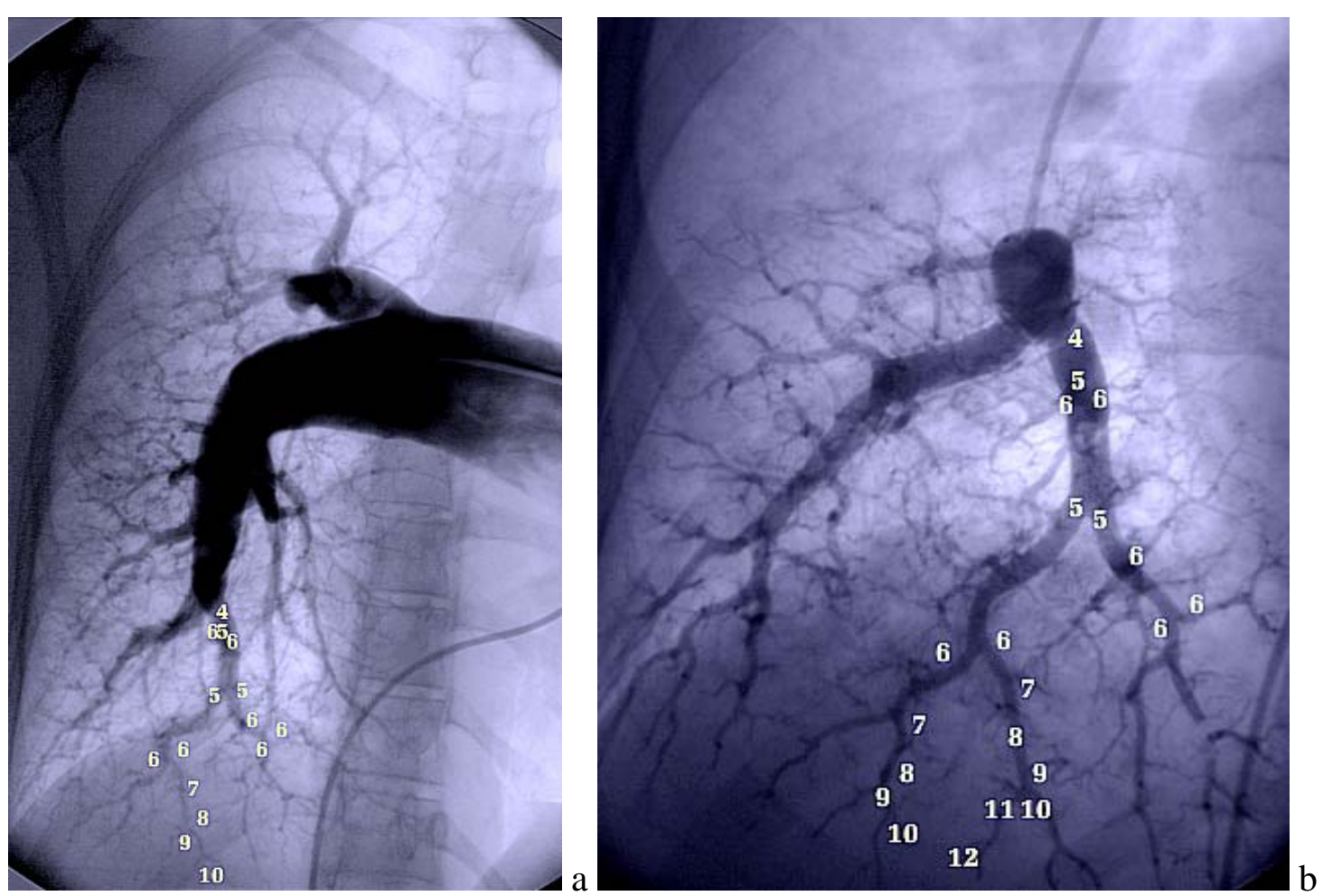

Abb. 3: Zählung der Aufzweigungsgenerationen in einer Segmentarterie des rechten Unterlappens in der Übersichtsangiographie (a) und in der Ballon-Okklusionsangiographie (b)

Bei der Auszählung der Aufzweigungsgenerationen entsprachen die Segmentarterien grundsätzlich Gefäßen 4. Ordnung, da proximal davon Lappenarterien, Hauptstämme für die rechte und die linke Lunge sowie der Truncus pulmonalis gelegen sind. Der Erfassung der weiteren Aufzweigungen distal der Segmentarterien wurde das Modell der irregulären Dichotomie (Duncker, 1985) zugrundegelegt (vgl. 1.1). Der Sprung in eine höhere Aufzweigungsgeneration erfolgte also erst bei der ersten gleichwertigen Gabelung eines Gefäßes, und nicht gleichwertige Seitäste dieses Gefäßes wurden ebenfalls der nächsthöheren Ordnung zugerechnet (Abb. 4).

Abb. 4 zeigt die Berücksichtigung kaliberschwacher, überzähliger Abzweigungen. Die Subsegmentarterie (5) teilt sich also in drei Arterien 6. Ordnung auf.

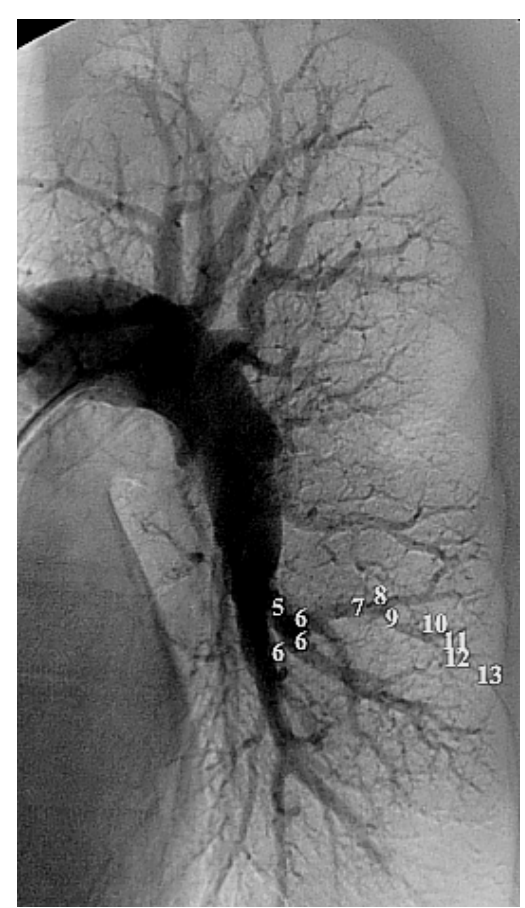


Im nächsten Schritt wurden die Übersichtsangiographien auf das Vorhandensein thrombembolischer Residuen überprüft. Dabei kann es sich um Füllungsdefekte, Stenosen, Verschlüsse, Kalibersprünge, Wandauflagerungen und Gefäßektasien handeln. Es erfolgte eine seitengetrennte Dokumentation der Befunde auf Hauptstamm-, Lappenarterien- und Segmentarterienniveau. Innerhalb der Segmentarterien wurde eine generelle Auszählung der thrombembolisch befallenen Gefäße vorgenommen und darüber hinaus die Anzahl der verschlossenen Segmentarterien bestimmt. Die weiter peripher gelegenen thrombembolisch bedingten Veränderungen wurden jeweils dem Lappen zugeordnet und die Angabe erfolgte als Anzahl befallener Lappen.

Die Parenchymminderperfusion wurde an übersteuerten Bildern aus der Parenchymphase der Übersichtsangiographie semiquantitativ bestimmt.

\subsubsection{Ballon-Okklusionsangiographie}

Die Analyse der Ballon-Okklusionsangiographie erfolgte anhand der Parameter Anzahl der dargestellten Gefäße (Abb. 5), Kalibersprung (Abb. 5), Stenose (Abb. 6), Webs und Bands bzw. thrombembolisch bedingte Füllungsdefekte (Abb. 7) und Verschluss (Abb. 7).
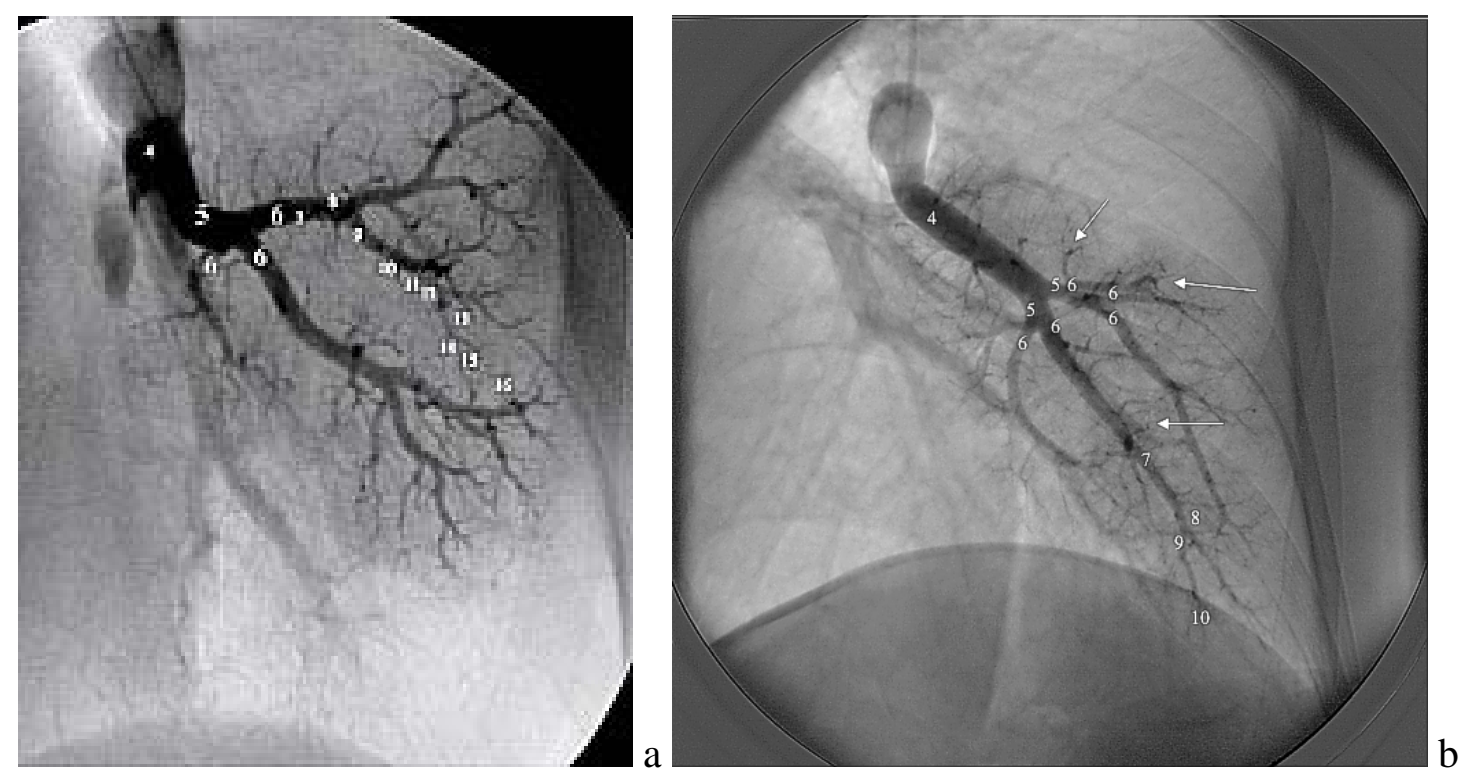

Abb. 5: a) Abzählung der in der Ballon-Okklusionsangiographie dargestellten Gefäße (gleicher Patient wie Abb. 4), b) Kalibersprünge in den peripheren Gefäßen (Pfeile) 

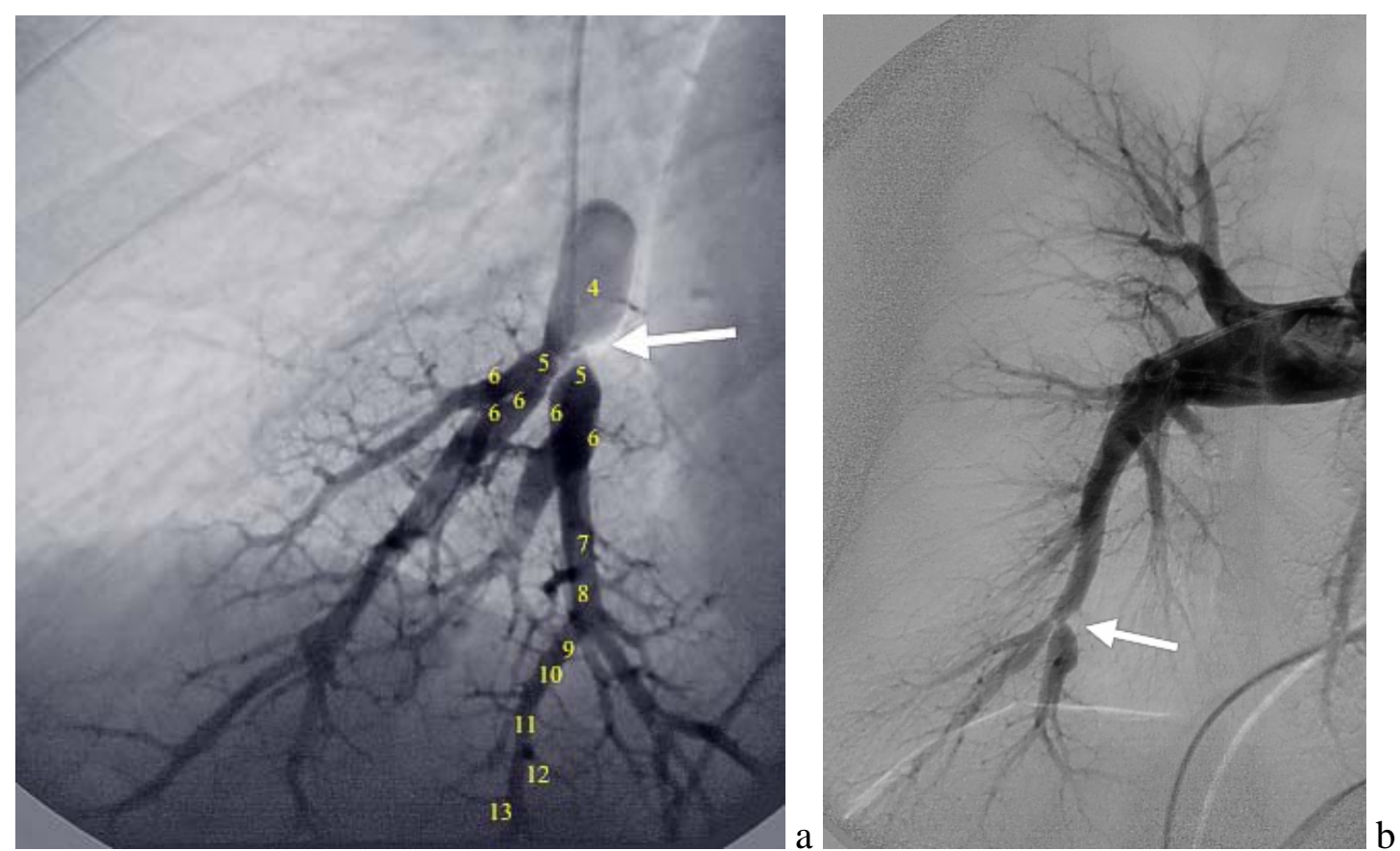

Abb. 6: Abgangstenose einer Subsegmentarterie im posterobasalen Unterlappensegment rechts in der Ballon-Okklusionsangiographie (a) und in der Übersichtsangiographie (b)
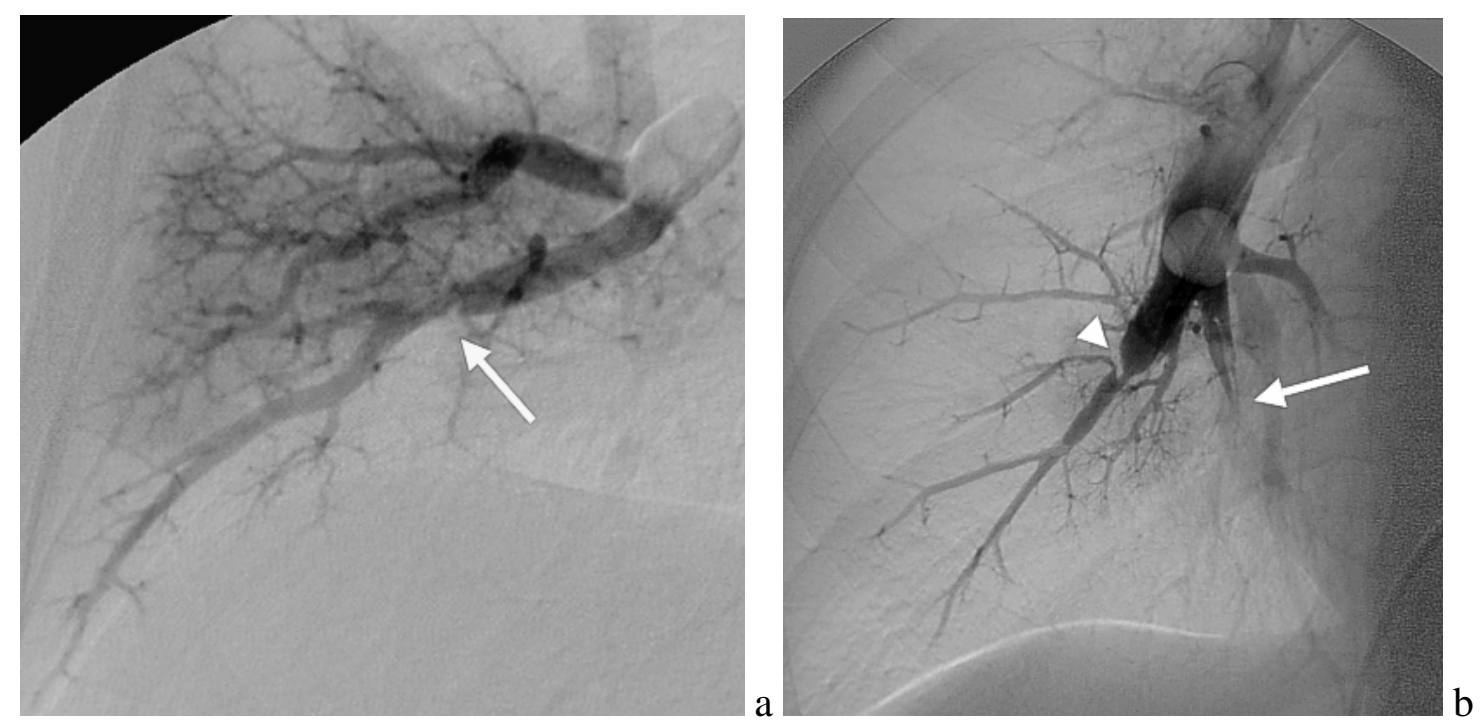

Abb. 7: a) Webs und Bands in einer Subsegmentarterie (Pfeil) des Mittellappens, b) Stenose (Pfeilspitze) sowie Web mit nachfolgendem Verschluss in Subsegmentarterien des rechten Unterlappens (dicker Pfeil)

Dabei wurde jedes Merkmal auf Höhe der Segmentarterien, der Subsegmentarterien, der Gefäße 6. Ordnung und der davon peripher gelegenen Gefäße überprüft. Da die kleinsten peripheren Gefäße kaum auszählbar sind, musste die Angabe hier semiquantitativ beschränkt werden auf „,<5“ oder „ $\geq 5$ “. Jedes in der Okklusionsangiographie detektierte Merkmal wurde dann daraufhin überprüft, ob es in der Übersichtsangiographie wiederzufinden ist. 
Außerdem wurde die Aufzweigungsmorphologie des dargestellten Gefäßbaumes qualitativ beurteilt. Neben der normalen Anatomie wurde das Vorliegen stark verplumpter und gewundener Gefäße als „plumpe Rettichwurzel“ (Abb. 8) bezeichnet und das Erscheinungsbild eines Baumes mit wenigen, dünnen Ästen als „dürre Birke“ (Abb. 8). Die Registrierung der Merkmale erfolgte in diesem Falle nur qualitativ in Form von vorhanden (ja) oder nicht vorhanden (nein).
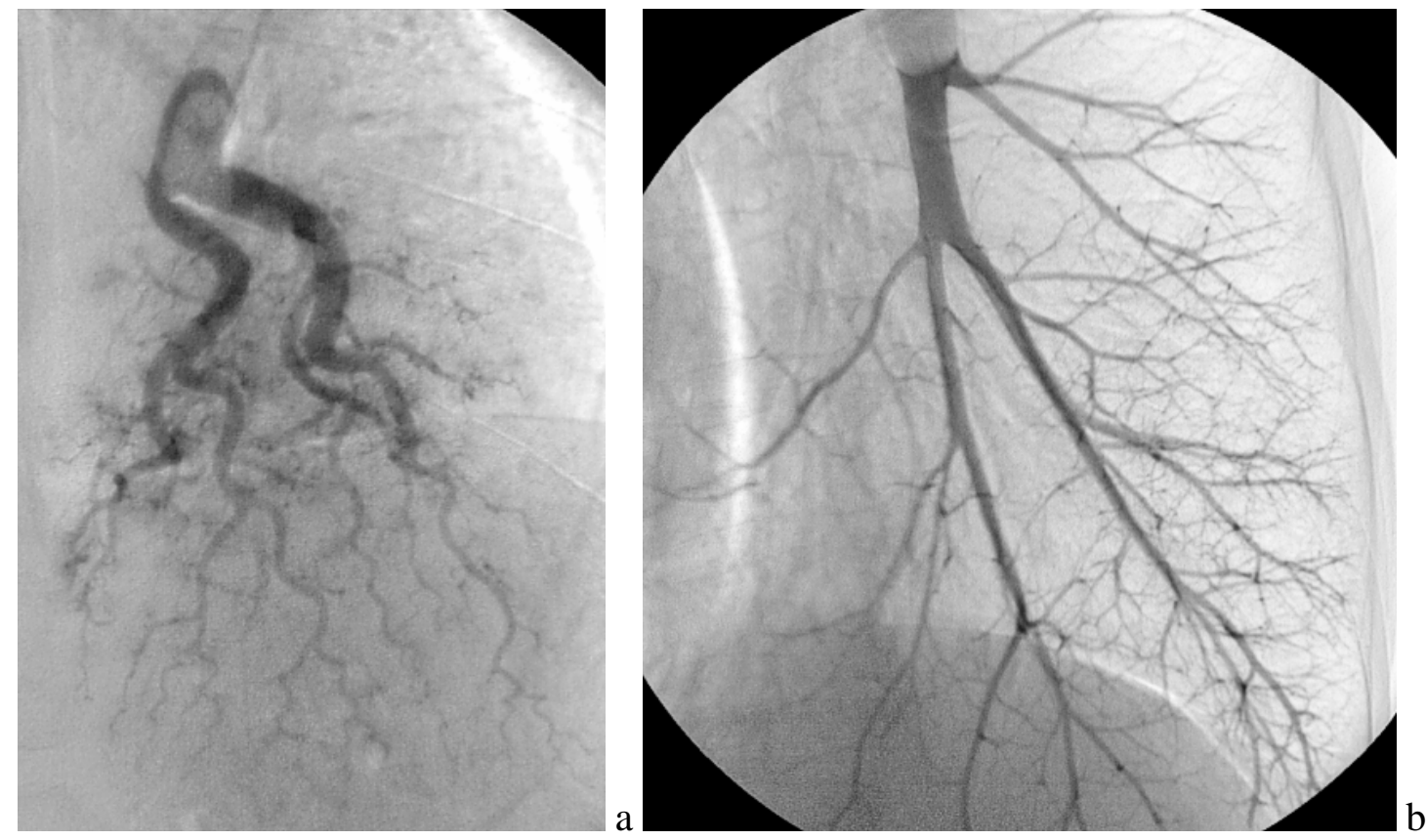

Abb. 8: a) korkenzieherartig gewundener Verlaufstyp der peripheren Lungengefäße (plumpe Rettichwurzel), b) gestreckter Gefäßverlauf mit spärlichen Verzweigungen (dürre Birke)

Kollateralen $\mathrm{zu}$ Pulmonalgefäßen in benachbarten Lungensegmenten oder Anastomosen zu pleuralen Bronchialarterien wurden ausgezählt (Abb. 9, S. 22). 

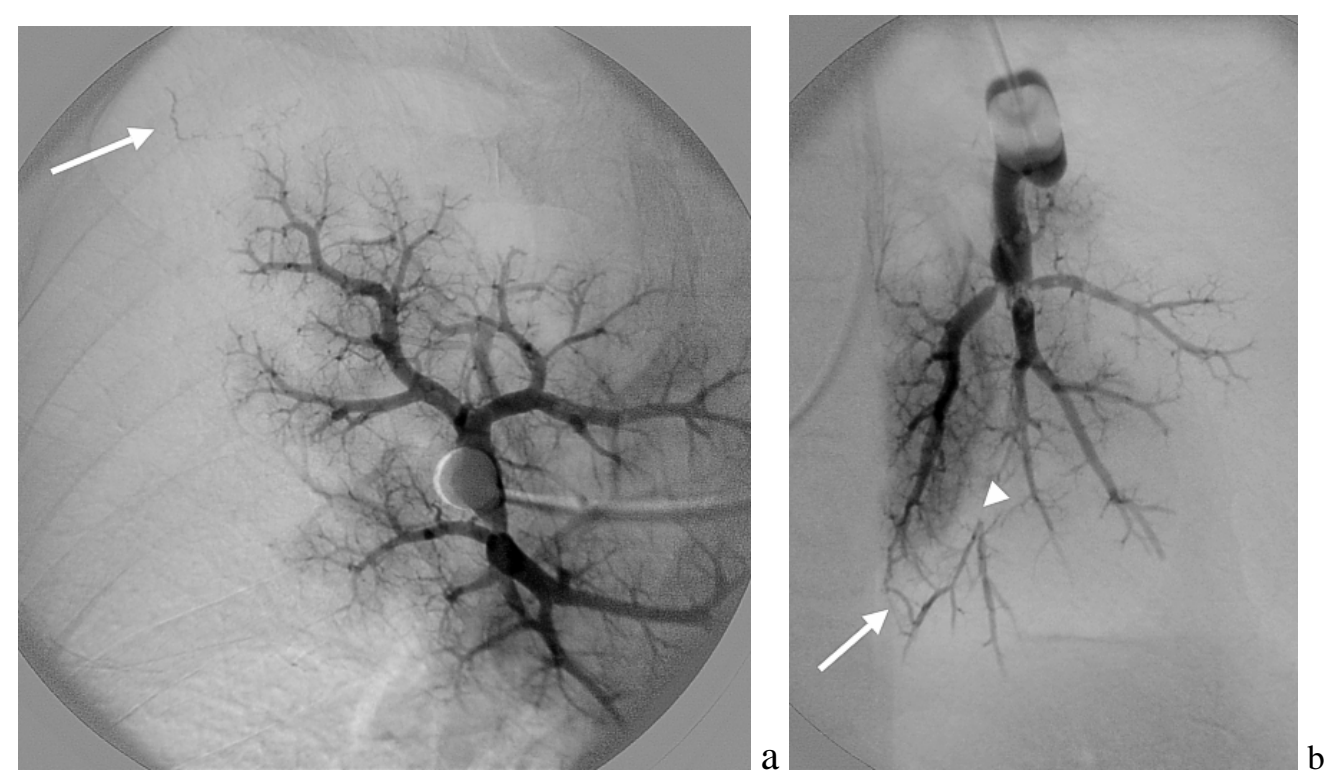

Abb. 9: a) Kollaterale zu einer subpleuralen Bronchialarterie (Pfeil), b) Kollaterale $\mathrm{zu}$ einem Nachbarsubsegment (Pfeil) mit Wiederauffüllung eines zentral verschlossenen Pulmonalgefäßes

Abschließend wurde die Anfärbung des dargestellten Parenchymes beurteilt (normal/ reduziert/ fehlt) und in Beziehung gesetzt zur Kontrastierung der drainierenden Pulmonalvenen (normal/ reduziert/ fehlt) (Abb. 10).

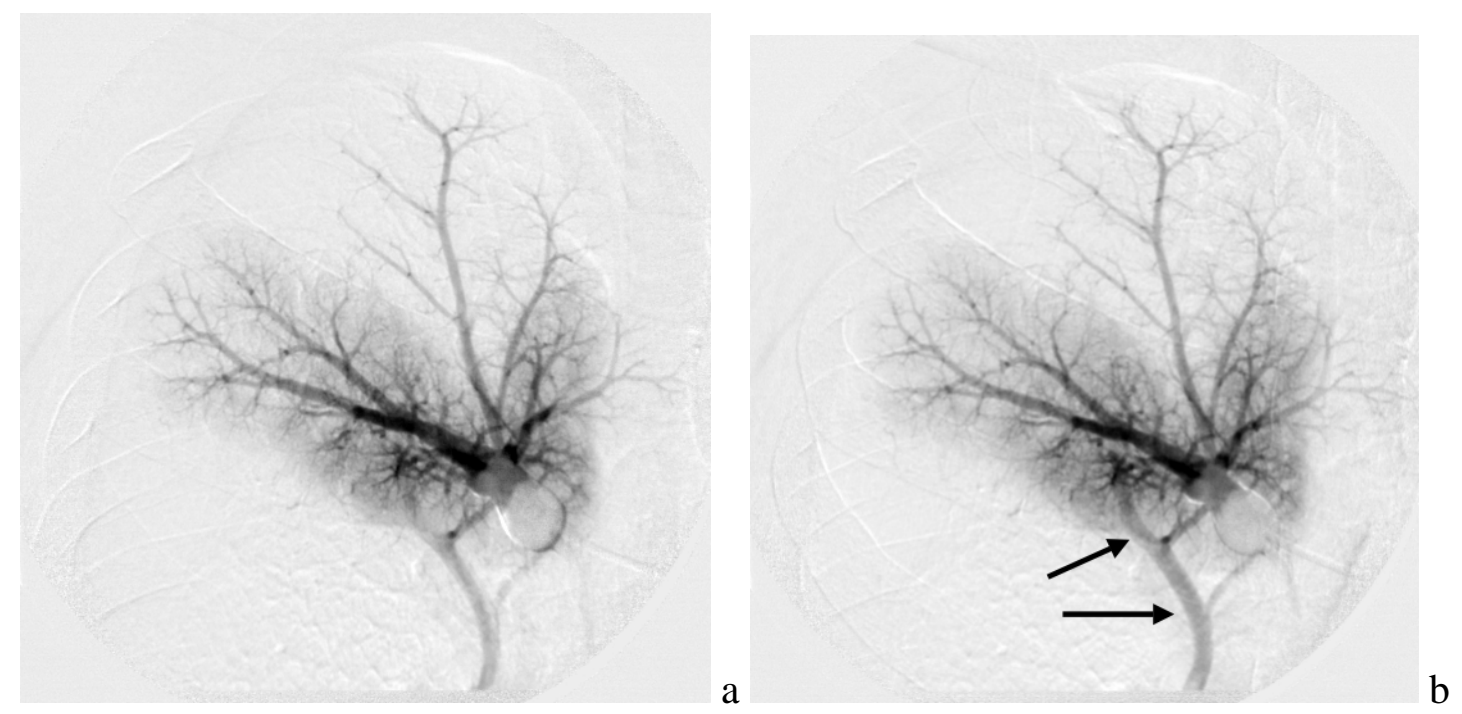

Abb. 10: a) regional reduzierte Parenchymanfärbung bei peripherer Embolie im Oberlappen rechts, b) zeitgerechte Venenfüllung aus den normal angefärbten Parenchymabschnitten

Aus der Krankenakte wurden die Befunde der innerhalb des gleichen stationären Aufenthaltes durchgeführten Ventilations-Perfusions-Szintigraphie und der Rechtsherzkatheteruntersuchung entnommen. 
Im weiteren Verlauf wurde verfolgt, bei welchen Patienten die Indikation zur PTEA gestellt wurde und bei welchen Patienten die Operation tatsächlich durchgeführt wurde.

Insgesamt wurden also für jeden Patienten klinische, radiologische und funktionelle Merkmale registriert. Die Daten wurden in eine Excel-Tabelle eingetragen.

\subsection{Statistische Auswertung}

Die statistische Auswertung der Daten wurde unter Zuhilfenahme verschiedener Funktionen der Statistik-Software $\mathrm{R}$ in der Version 1.9.1 (R Development Core Team (2004)) vorgenommen. Mit Hilfe von Microsoft Excel wurden einige der grafischen Darstellungen angefertigt. Es kamen folgende statistische Verfahren zum Einsatz:

\subsubsection{Deskriptive univariate Methoden}

Für die univariate (= eindimensionale) explorative Datenanalyse (= univariate EDA) eines jeden der stetigen Merkmale Alter, Erkrankungsdauer, Minderperfusion rechts bzw. links, mittlerer pulmonalarterieller Druck (mPAP), gezählte Aufzweigungsgenerationen und gezählte Merkmale in der Angiographie wurden sowohl Dotcharts als auch Boxplots der beobachteten Daten (gegebenenfalls nach gewissen Kriterien gruppiert) angefertigt. Dies geschah, um einerseits mittels der Dotcharts die Originaldaten (= Rohwerte) ohne Informationsverlust darzustellen (Geßler, 1993) und andererseits durch die Boxplots eine kompakte Präsentation der zentralen Lage und der Streuung eines Datensatzes basierend auf Minimum, 1. Quartil, Median, 3. Quartil und Maximum der Daten zu bieten. Boxplots bestehen aus einer zentralen „Box“, die sich vom 1. bis zum 3. Quartil der Daten erstreckt und somit die zentralen 50 \% der Daten umfasst. Die Box enthält eine Linie, welche den Median der Daten markiert. Unter- und oberhalb der Box erstrecken sich gestrichelte Linien bis $\mathrm{zu}$ den extremsten, jedoch noch nicht als potenzielle Ausreißer geltenden Daten. Als potenzieller Ausreißer gelten Beobachtungen, die weiter als der 1,5-fache Quartilsabstand (= 1,5* (3. Quartil - 1. Quartil)) vom 1. 
Quartil nach unten oder vom 3. Quartil nach oben entfernt sind; sie werden durch separate Symbole gesondert gekennzeichnet (Geßler, 1993).

Ferner wurden QQ-Plots als grafisch-statistische Hilfsmittel eingesetzt, um für jedes stetige Merkmal die Zulässigkeit der Annahme einer Normalverteilung der Daten zu untersuchen. Diese Plots ermöglichen den Vergleich der empirischen (Daten-)Verteilung mit einer theoretischen Verteilung, hier einer Normalverteilung. Dazu werden die aufsteigend sortierten Daten, d. h. die empirischen Quantile (auf der Ordinate) gegen die entsprechenden theoretischen Quantile der Standardnormalverteilung (auf der Abszisse) aufgetragen. Sind die Werte normalverteilt, befinden sich die Punkte in etwa entlang einer Geraden. Diese Soll-Gerade ist in den QQ-Plots jeweils eingezeichnet. Weichen die Punkte deutlich von dieser Gerade ab, so besteht eine Diskrepanz zwischen der postulierten Normalverteilung und der tatsächlichen Verteilung der Werte.

Für die Darstellung absoluter Häufigkeiten der beobachteten Ausprägungen diskreter (nominaler, ordinaler oder Zähl-) Variablen wurden Balkendiagramme als "grafische Häufigkeitstabellen" verwendet.

\subsubsection{Inferenzstatistische univariate Methoden}

Vergleiche zwischen OA und ÜA auf Basis merkmalspezifischer Untersuchungsergebnisse wurden pro Merkmal auf zweierlei Arten angestellt:

Einerseits qualitativ durch die Schätzung der Erfolgswahrscheinlichkeit, dass die Ballon-Okklusionsangiographie für das betrachtete Merkmal schlicht „mehr zeigt“ als die Übersichtsangiographie, sowie andererseits quantitativ, indem beim betrachteten Merkmal die mittlere absolute Anzahl der in der OA entdeckten "Phänomene" mit derjenigen in der ÜA verglichen wurde.

Die Schätzwerte der Erfolgswahrscheinlichkeiten wurden im Rahmen passender Binomialmodelle durch relative Häufigkeiten bestimmt und mit einseitigen Konfidenzintervallen zum Niveau 95 \% versehen (gemäß des Verfahrens von Clopper und Pearson; vgl. Sachs L, 2004). Diese Intervalle sind insofern „einseitig“, als dass nur ihre Untergrenze berechnet wurde und ihre Obergrenze der theoretisch maximale Wert für eine Wahrscheinlichkeit, nämlich 1, ist. (Die hierfür verwendete R-Funktion ist binom.test() mit einseitiger Alternative.) 
Die Vergleiche von OA mit ÜA hinsichtlich absoluter Anzahlen entdeckter "Phänomene" geschahen auf Basis ihrer paarweisen Anzahldifferenzen und wurden mittels des gepaarten t-Tests (mit einseitiger Alternative) durchgeführt (da davon ausgegangen werden kann, dass die hierbei zum Einsatz kommende Teststatistik für die vorliegenden Stichprobenumfänge approximativ t-verteilt ist). Für die mittleren Differenzen wurden auch approximative einseitige 95 \%-Konfidenzintervalle angegeben. (Die relevante R-Funktion war t.test() mit einseitiger Alternative.)

Sonstige Mittelwert- bzw. Median-Vergleiche für zwei unabhängige Stichproben wurden ebenfalls mit Hilfe des t-Tests bzw. Wilcoxons Rangsummentest durchgeführt (Trampisch \& Windeler, 2000). Im Fall abhängiger Stichproben (weil z. B. die $\mathrm{zu}$ vergleichenden Werte paarweise am selben Patienten bestimmt wurden) kamen die Versionen dieser Tests für gepaarte Daten zum Einsatz.

Der Einsatz von Wilcoxons Rangsummentest für den Medianvergleich ist (insbesondere) bei Zweifeln an der Zulässigkeit einer Normalverteilungsannahme angezeigt, denn dann sollten inferenzstatistische Analysen mit Hilfe nichtparametrischer Methoden (wie eben z. B. Wilcoxons Rangsummentest) durchgeführt werden, da sie der Normalverteilungsannahme nicht bedürfen und somit allgemeiner einsetzbar sind. Der Preis hierfür ist im Fall der Gültigkeit der Normalverteilungsannahme ein (leichter) Verlust in der statistischen Güte (d. h. in der Fähigkeit, Lokationsunterschiede zu entdecken), der aber nicht von praktischer Relevanz ist, wenn die verwendeten Stichprobenumfänge "hinreichend" groß sind (Bortz et al., 2000).

\subsubsection{Deskriptive bivariate Methoden}

Für die Darstellung absoluter Häufigkeiten der beobachteten paarweisen Ausprägungen zweier diskreter (nominaler, ordinaler oder Zähl-) Variablen wurden multiple Balkendiagramme als "grafische bivariate Häufigkeitstabellen" angefertigt.

\subsubsection{Inferenzstatistische bivariate Methoden}

Tests auf Unabhängigkeit zweier Merkmale wurden mittels des Pearsonschen $\chi^{2}$ Unabhängigkeitstests durchgeführt. Dazu wurde für jedes der Merkmalspaare die 
Kontingenztafel (= Kreuztabelle) erstellt und die Hypothese getestet, dass die beiden Merkmale unabhängig sind (Weiß, 1999). Die $\chi^{2}$-Verteiltheit der verwendeten Pearsonschen $\chi^{2}$-Teststatistik gilt unter der Unabhängigkeitshypothese nur asymptotisch (d. h. für hinreichend große Stichprobenumfänge) und unter der Bedingung, dass in der Kontingenztafel der zwei jeweils betrachteten Merkmale alle erwarteten Einträge (= Zellhäufigkeiten) hinreichend groß sind (d. h. mindestens 5). Damit gelten die dokumentierten p-Werte auch hier nur approximativ.

Die Untersuchung der Beziehung zwischen zwei Einflussfaktoren A und B geschah einerseits mittels Pearsons $\chi^{2}$-Test auf Unabhängigkeit (wann immer möglich, d. h., wenn in der jeweiligen 2x2-Tafel alle unter Unabhängigkeit erwarteten Zellhäufigkeiten größer als 5 waren) und andererseits - entweder als Ersatz oder als Bestätigung - mittels Fishers exaktem Test auf (bedingte) Unabhängigkeit (falls die unter Unabhängigkeit erwarteten Zellhäufigkeiten in der betreffenden 2x2-Tafel zu niedrig waren) (Weiß, 1999). (Die obigen Tests wurden durch die R-Funktionen chisq.test() und fisher.test() realisiert.)

\subsubsection{Prognosemodell}

Für die Schätzung der (bedingten) Wahrscheinlichkeit, dass unter der Bedingung, in der Übersichtsangiographie (ÜA) keine thrombembolischen Residuen (tR) entdeckt zu haben, auch in der Okklusionsangiographie (OA) keine gefunden werden (was wir damit gleichsetzen, dass tatsächlich keine vorhanden sind), kommt ein wahrscheinlichkeitstheoretisches Modell zur Anwendung. Dessen Herleitung und Ergebnisse sind im folgenden Kapitel (vgl. 3.6) erläutert. 


\subsection{Patienten}

Es wurden 50 Pulmonalisangiographien von 50 Patienten ausgewertet. Bei allen Patienten konnten in einer Sitzung jeweils eine konventionelle Übersichtsangiographie in zwei Ebenen und anschließend mindestens eine Ballon-Okklusionsangiographie (eine bis sechs) durchgeführt werden. Insgesamt waren damit 154 Okklusionsangiographien, im Mittel 3,08 pro Patient, auszuwerten.

Die Grafik 1 (Boxplot vgl. 2.6.1) beschreibt die Altersverteilung des Studienkollektives. Der Mittelwert beträgt 55,96 Jahre, der Median (vertikaler Strich in der Box) liegt bei 59 Jahren. Die Endpunkte markieren den jüngsten Patienten (27 Jahre) und den ältesten Patienten (74 Jahre). Die Hälfte der Patienten war zwischen 49 und 64 Jahre alt.

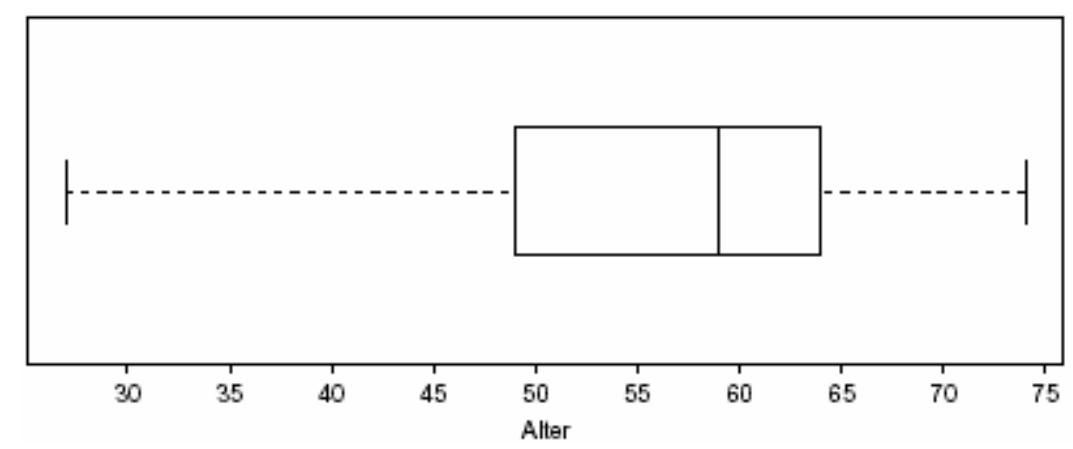

Grafik 1: Altersverteilung im Studienkollektiv

Die Grafik 2 informiert über die Dauer der Erkrankung Chronische Pulmonale Hypertonie bei den Patienten im Studienkollektiv. Der Median liegt bei zwei Jahren, die Daten streuen von sechs Monaten bis zu 27 Jahren. Dreiviertel aller Patienten hatten eine Erkrankungsdauer von höchstens vier Monaten.

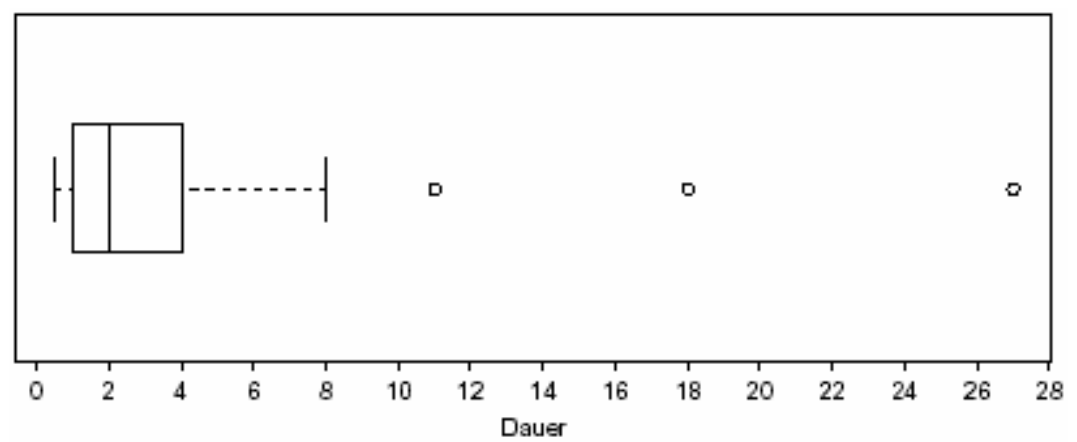

Grafik 2: Dauer der Erkrankung 
Die Balkendiagramme 1a) und 1b) führen Patienten auf, bei denen die Indikation zur pulmonalen Thrombendarteriektomie (PTEA) gestellt wurde (a) und jene, bei denen die Operation tatsächlich durchgeführt wurde. In Diagramm b) kommt die Gesamtpatientenzahl von 48 (anstelle von 50) dadurch zustande, dass bei zwei Patienten mit prinzipiell vorliegender Indikation zur Operation nicht eruierbar war, ob der Eingriff tatsächlich durchgeführt wurde.
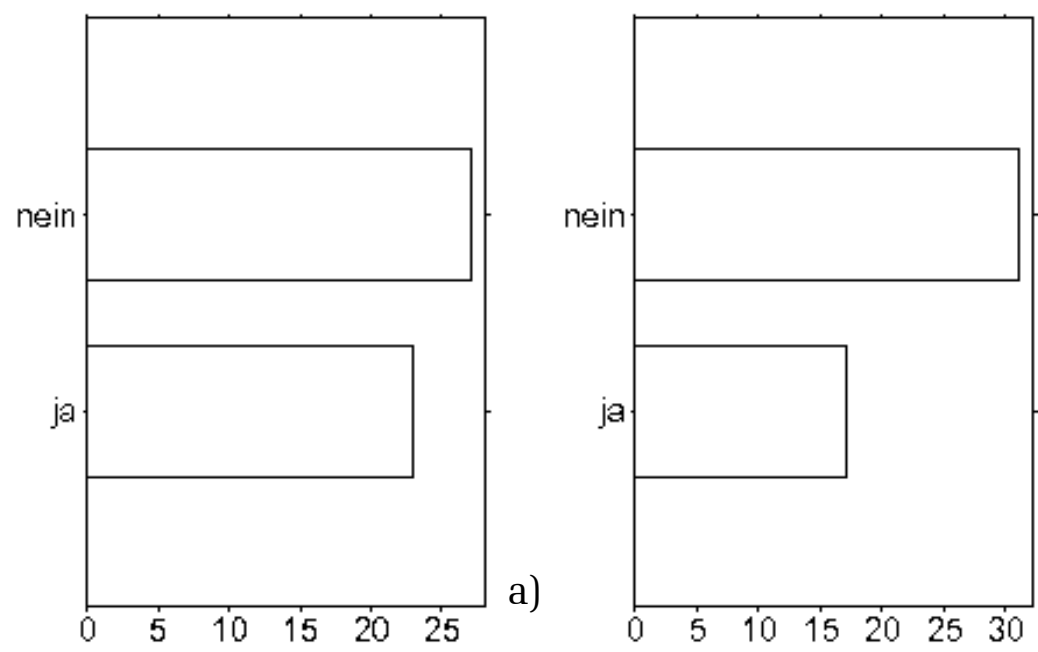

b)

Diag. 1: Indikation zu pulmonalen Thrombendarteriektomie (PTEA)

Die pulmonale Thrombendarteriektomie (PTEA) wird nur bei Patienten mit CTEPH und relativ weit zentral gelegenen Residuen durchgeführt. Im Studienkollektiv waren das 17 von 35 Patienten (48,5\%, Diagramm 2).

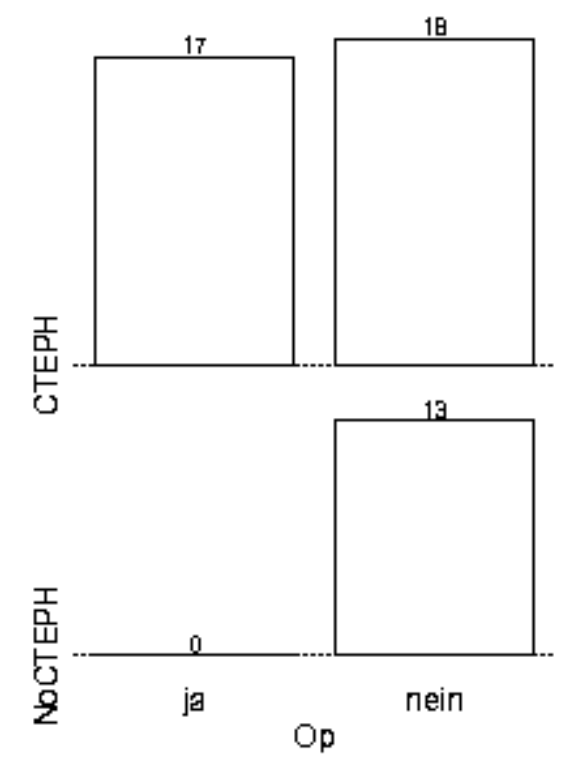

Diag. 2: Anzahl der Operationen (PTEA) 
Die Grafik 3 zeigt die Verteilung des mittleren pulmonalarteriellen Druckes (mPAP) im Studienkollektiv zum Zeitpunkt der Pulmonalisangiographie. Mittelwert (50,45 mmHg) und Median (vertikale Linie innerhalb der Box bei $50 \mathrm{mmHg}$ ) entsprechen nahezu einander. Die Werte liegen zwischen dem Minimum von 19 und dem Maximum von $94 \mathrm{mmHg}$.

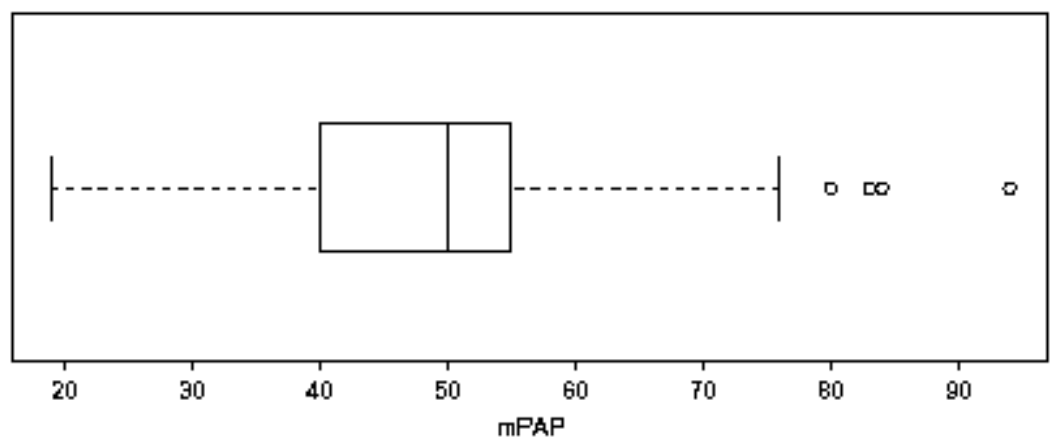

Grafik 3: mittlerer pulmonalarterieller Druck im Studienkollektiv

\subsection{Angiographie und Szintigraphie}

Das Diagramm 3 zeigt in a), dass bei 36 Patienten in der Zusammenschau von Übersichtsangiographie und Ballon-Okklusionsangiographie mindestens ein thrombembolisches Residuum (Webs und Bands, Stenosen, Verschlüsse $=$ Throm) zu finden war. In Diag. 3b) ist die Häufigkeit der bezüglich Thrombembolien positiven Szintigraphien $(n=37)$ gegenübergestellt.
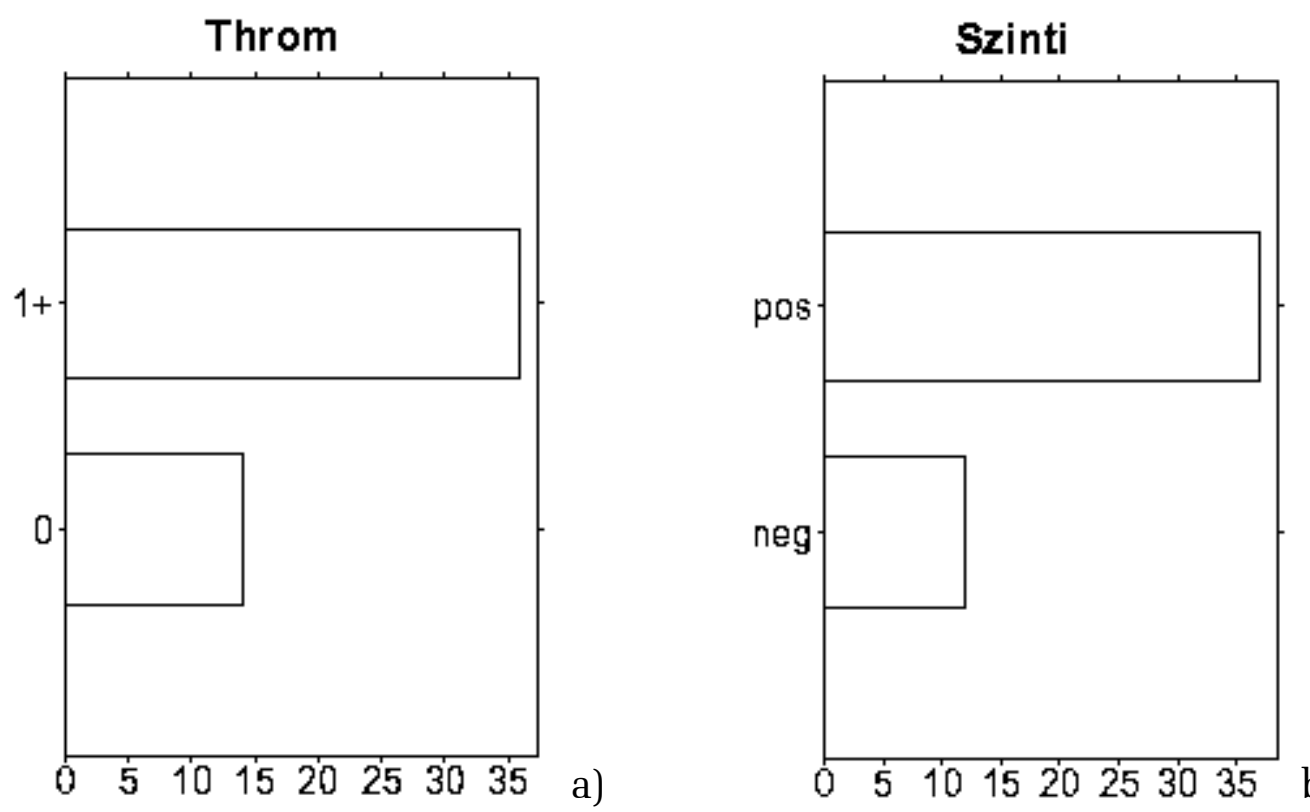

b)

Diag. 3: thrombembolische Residuen in Angiographie und Szintigraphie 
In den Diagrammen 4a) und 4b) sind die hinsichtlich thrombembolischer Veränderungen positiven Untersuchungsverfahren Angiographie (Throm) und Szintigraphie nach Diagnosen CTEPH versus NoCTEPH aufgetragen. In der Angiographie wurde somit ein Patient als falsch negativ eingestuft. 36 Patienten wurden als richtig positiv eingestuft $(97,3 \%)$. Hingegen fanden sich in der Szintigraphie zwei falsch negative und drei falsch positive Befunde. In 34 Fällen (91,9\%) war die Untersuchung richtig positiv hinsichtlich des Vorliegens einer Lungenembolie. Die Unterscheidung zwischen den Diagnosen CTEPH und No-CTEPH erwies sich als signifikant abhängig von den Ergebnissen aus Angiographie (Fishers exakter Test: $\mathrm{p}<0,0001$ ) und Szintigraphie (Fishers exakter Test: $\mathrm{p}<0,0001$ ). Die Patienten, die eine pulmonale Hypertonie aufgrund anderer Ursachen hatten (No-CTEPH), zeigten alle auch in der Pulmonalisangiographie einen negativen Befund (100\% richtig negative Befunde). In der Grafik für die Szintigraphie finden sich nur 36 CTEPHPatienten, da für einen Patienten kein Szintigraphiebefund vorlag.

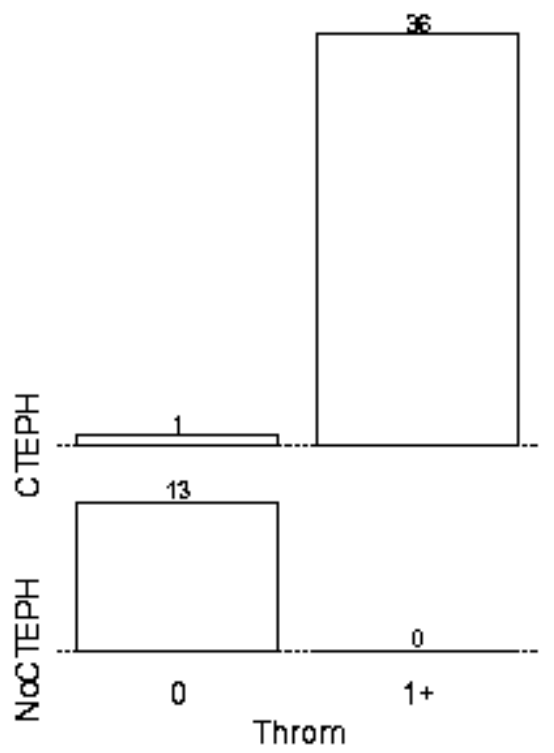

a)

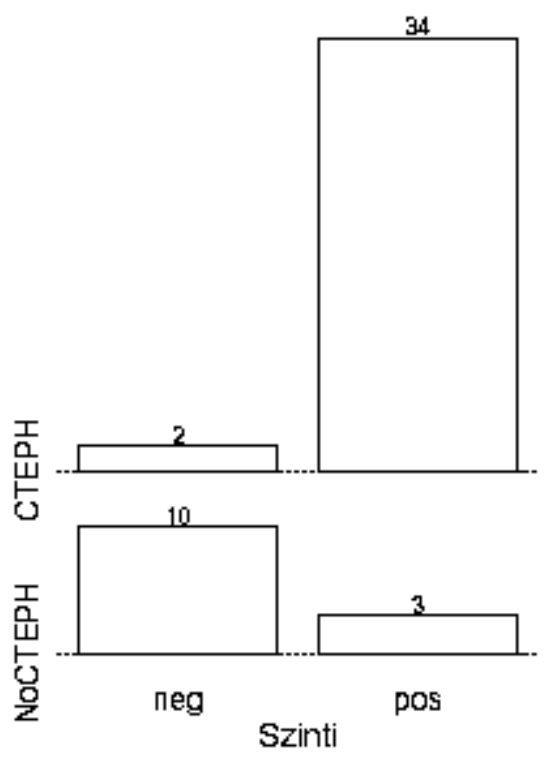

Diag. 4: Sensitivitäten und Spezifitäten der Untersuchungsverfahren b) Szintigraphie und Angiographie im Studienkollektiv

Die Beziehung der Untersuchungsbefunde aus Angiographie und Szintigraphie ist im Diagramm 5 verdeutlicht. Es sind nur 35 Patienten mit positiver Angiographie aufgeführt, da für einen Patienten (wie oben bereits erwähnt) kein Szintigraphiebefund vorlag. Zwei von 35 Patienten mit positiver Angiographie hatten ein bezüglich thrombembolischer Residuen negatives Szintigraphieergebnis. Im Test ergibt sich eine hochsignifikante Abhängigkeit der Untersuchungsverfahren 
(Fishers exakter Test: $\mathrm{p}<0,0001$ ). Dies bedeutet in Zusammenschau mit Diagramm 5, dass bei positivem Ausgang der Szintigraphie auch die Angiographie mit hoher Wahrscheinlichkeit thrombembolische Veränderungen zeigt.

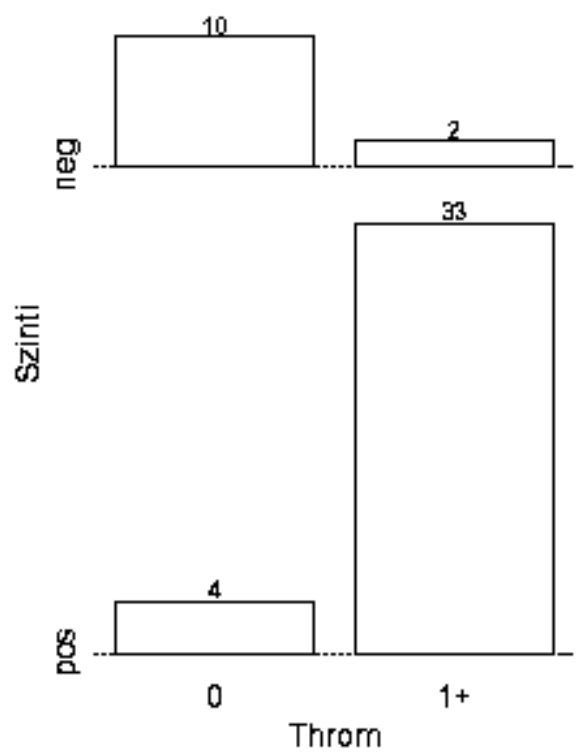

Diag 5: Emboliefolgen in Angiographie und Szintigraphie

\subsection{Parenchymminderperfusion}

Grafik 4 verdeutlicht das Ausmaß der Parenchymminderperfusion aller 50 Patienten seitengetrennt rechts (a) und links (b). Die mittlere Parenchymminderperfusion des Gesamtkollektives zeigt keinen signifikanten Unterschied zwischen rechter und linker Lunge (gepaarter $\mathrm{t}$-Test, $\mathrm{p}=0,41$ ).

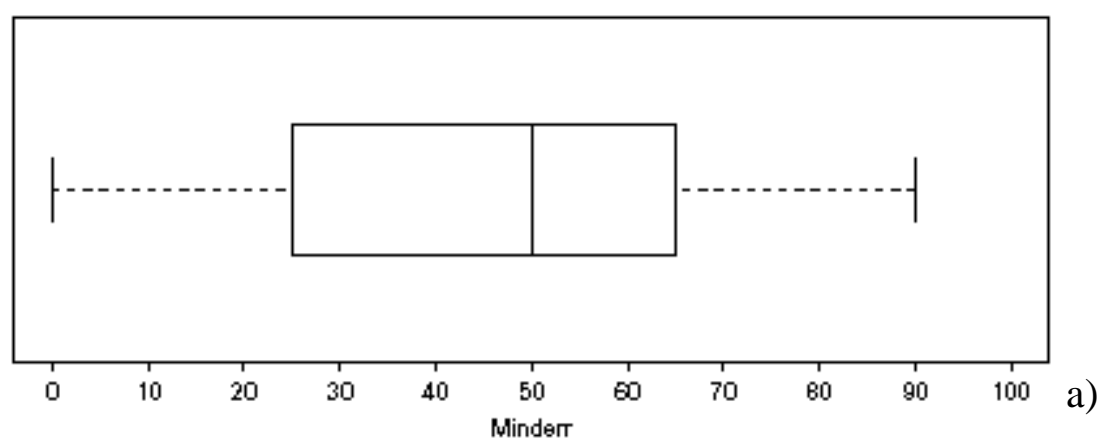




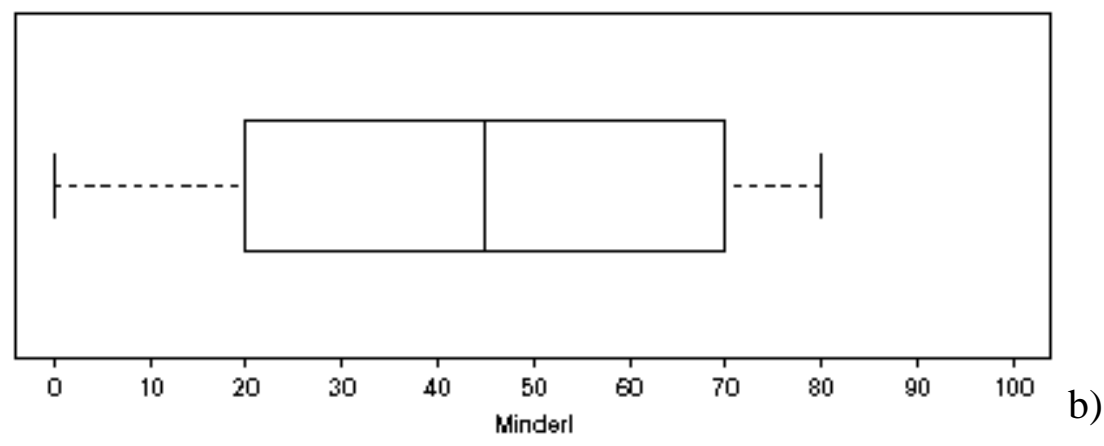

Grafik 4: Parenchymminderperfusion der rechten (a) und der linken Lunge (b)

Die Differenzen der Minderperfusion von rechter und linker Lunge (Grafik 5) sind annähernd normalverteilt. Der t-Test kann also unzweifelhaft darauf angewendet werden. Die durchschnittliche Seitendifferenz der Minderperfusion im Gesamtkollektiv beträgt 2,8\% (mit 95\%-Konfidenzintervall [-3,92\%; 9,53\%]).
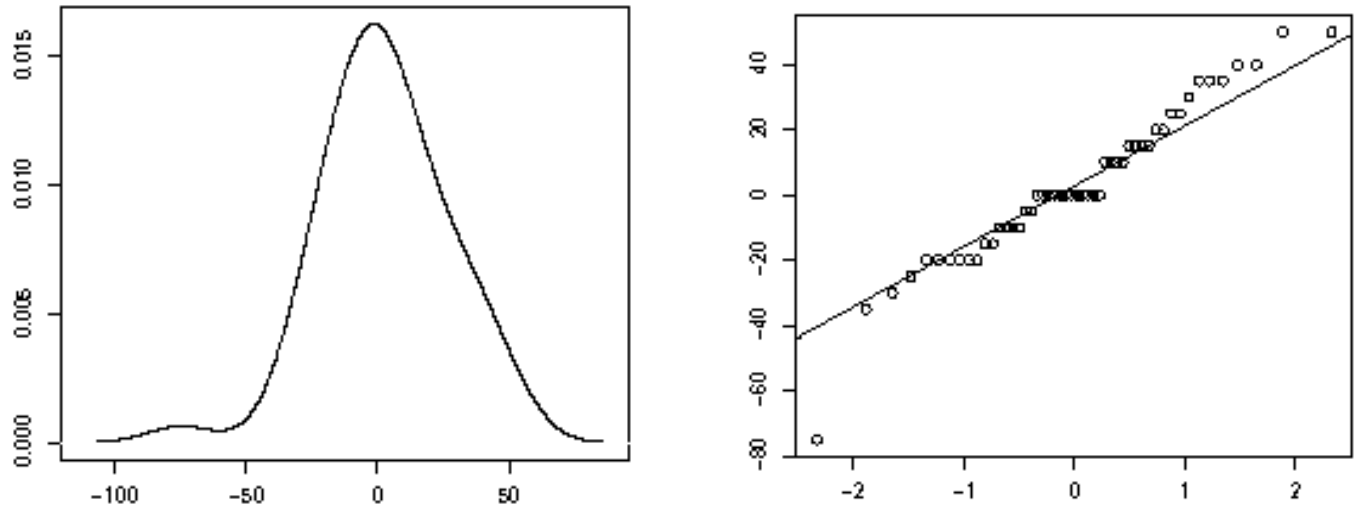

Grafik 5: Das geglättete Histogramm und der QQ-Plot (vgl. 2.6.1) der Differenzen zwischen den Parenchymminderperfusionen rechts und links zeigen, dass die Normalverteilungsannahme gerechtfertigt ist

Die mittlere Minderperfusion beider Lungen zusammengenommen im Studienkollektiv ist in Grafik 6 veranschaulicht. Der Mittelwert beträgt 44,8\% (mit 95\%-KI [37,6\%; 52,0\%]), der Median (durch vertikale Linie in der Box markiert) liegt bei $50 \%$.

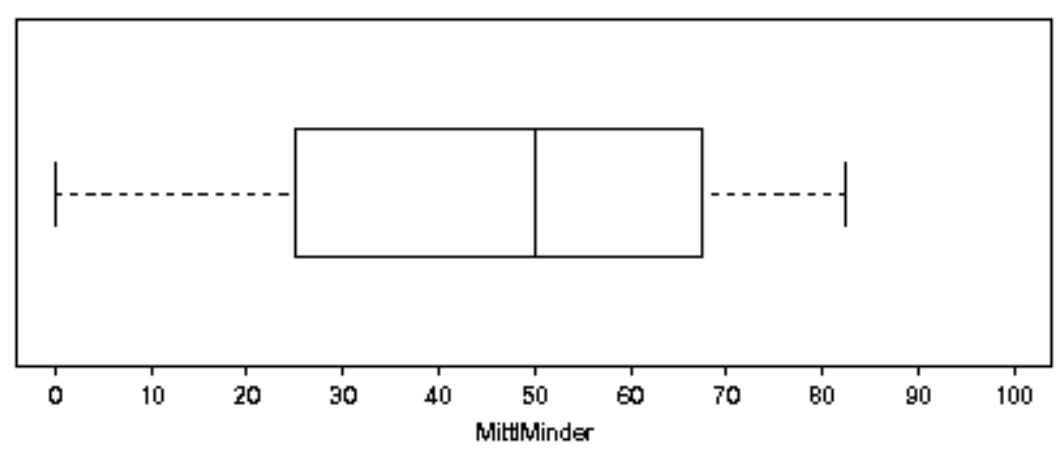

Grafik 6: mittlere Minderperfusion beider Lungen 
Bei der Trennung des Kollektives in CTEPH- und NoCTEPH-Patienten ergibt sich ein signifikanter Unterschied (Wilcoxon-Test, $p=0,0023$ ), wobei die Patienten mit einer chronischen thrombembolischen pulmonalen Hypertonie (CTEPH) eine deutlich ausgeprägtere Minderperfusion des Lungenparenchymes aufweisen:

CTEPH: Arithmet. Mittel $=51,1 \%$ und Median $=57,5 \%$.

NoCTEPH: Arithmet. Mittel $=27,0 \%$ und Median $=15 \%$.

Die beiden QQ-Plots in Grafik 7 zeigen eine asymmetrische Verteilung der mittleren Parenchymminderperfusion für die NoCTEPH- (a) und die CTEPHGruppe (b). Daher ist der t-Test unzulässig, und es kommt bei den vorangegangenen Berechnungen der Wilcoxon-Test zu Anwendung.
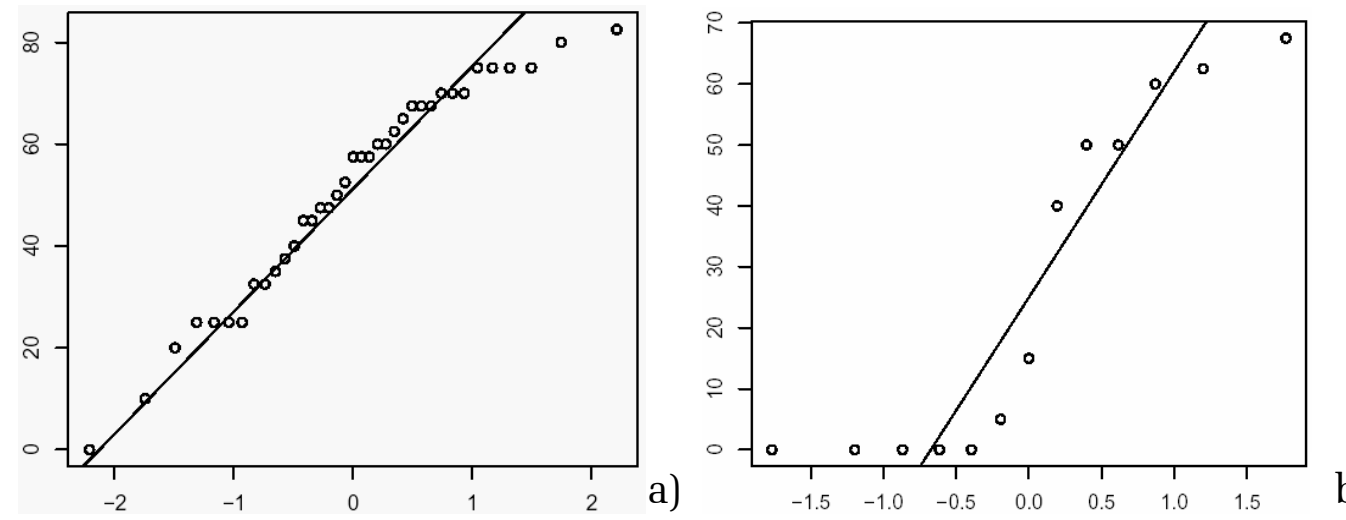

b)

Grafik 7: Die beiden QQ-Plots der mittleren Parenchymminderperfusion bei CTEPHPatienten (a) und No-CTEPH-Patienten (b) zeigen, dass die Annahme einer Normalverteilung zumindest für letztere unzulässig ist

Der Vergleich der Patienten, bei denen die Indikation zur pulmonalen Thrombendarteriektomie (OPInd $=$ ja) gestellt wurde, mit jenen, bei denen keine OP-Indikation (OPInd $=$ nein) bestand, zeigte ebenfalls einen hochsignifikanten Unterschied hinsichtlich der Mediane (hochsignifikanter Shift des Medians) der mittleren Parenchymminderperfusion. Hier lagen höhere Werte in der Gruppe der Patienten mit einer Indikation zur Operation vor (Wilcoxon-Test, $p=0,0112$ ):

$$
\begin{aligned}
& \text { OPInd }=\text { ja: Arithmet. } \text { Mittel }=54,9 \% \text { und Median }=60 \% . \\
& \text { OPInd }=\text { nein: Arithmet. Mittel }=36,2 \% \text { und Median }=40 \% .
\end{aligned}
$$

Innerhalb der CTEPH-Gruppe ergibt sich jedoch kein signifikanter Unterschied im Ausmaß der Minderperfusion zwischen Patienten mit OP-Indikation und Patienten ohne OP-Indikation: 
Mittlere Parenchymminderperfusion innerhalb der CTEPH-Gruppe:

OPInd $=$ ja: Arithmet. Mittel $=54,9 \%$ und Median $=60 \%$.

OPInd $=$ nein: Arithmet. Mittel $=44,8 \%$ und Median $=48,8 \%$.

Kein signifikanter Unterschied (t-Test: $p=0,16$; Wilcoxon: $p=0,15$ ).

Seitenvergleich der Parenchymminderperfusion innerh. der CTEPH-Gruppe:

"rechts minus links": Arithmet. Mittel = 6,2 \% ( Median $=0 \%$ ).

Kein signifikanter Unterschied (gepaarter t-Test: $\mathrm{p}=0,1461$ ).

Diagramm 6 zeigt die Ergebnisse der semiquantitativen Abschätzung von Parenchymanfärbung und Venenfüllung in der Ballon-Okklusionsangiographie. Die überwiegende Mehrzahl der Fälle zeigte eine diffuse oder regionale Reduktion der Parenchymanfärbung. Dreimal ergab sich ein Normalbefund (Abb. 11a).

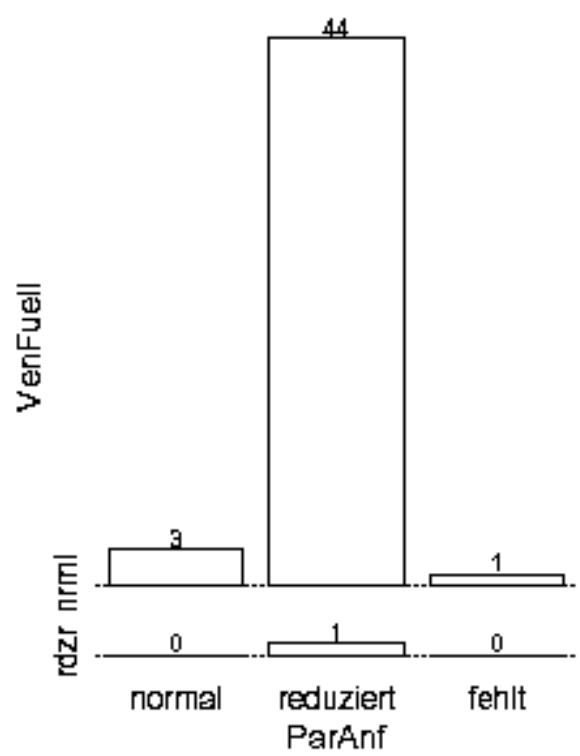

Diag. 6: Parenchymanfärbung und Venenfüllung in der Ballon-Okklusionsangiographie

In einem Fall machten wir die besondere Beobachtung, dass sich die Pulmonalarterien bis in die kleinsten Verästelungen hinein unauffällig darstellten und sich daraus ohne reguläre Anfärbung des Lungenparenchymes die Pulmonalvenen kräftig kontrastierten (Abb. 11b). Bei diesem Patienten konnte eine pulmonale kapilläre Hämangiomatose nach Lungenbiopsie histologisch gesichert werden. 

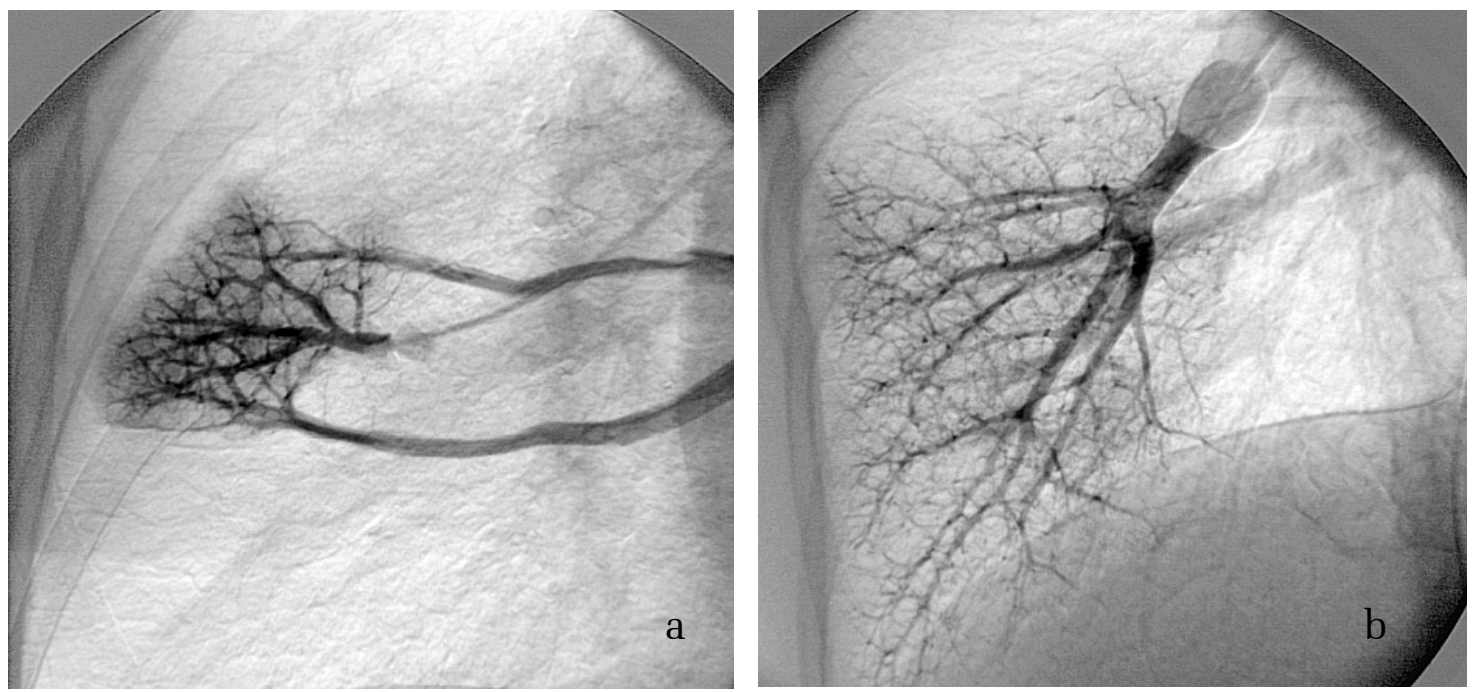

Abb. 11 : Verhältnis von Parenchymanfärbung und Venenfüllung in der Ballon-Okklusionsangiographie: a) Normalbefund, b) pulmonale kapilläre Hämangiomatose (PCH)

Diese Besonderheit veranlasste uns dazu, das Kontrollkollektiv (vgl. 2.1) ebenfalls auf dieses Phänomen hin zu untersuchen. Dabei fanden wir zwei Patienten mit pulmonaler venookklusiver Erkrankung (PVOD), die die gleiche Konstellation mit fehlender Parenchymanfärbung bei normaler Venenfüllung aufwiesen. Bei einem weiteren Patienten ist die Differentialdiagnose zwischen primärer pulmonaler Hypertonie (PPH) und PCH bzw. PVOD nicht bioptisch abgeklärt worden.

\subsection{Thrombembolische Residuen}

Die vier Säulendiagramme (Diagramm 7, S. 36) veranschaulichen das Auftreten thrombembolischer Residuen in Pulmonalarterienhauptstämmen (ThrHau), Lappenarterien (ThrLap), Segmentarterien rechts (ThrSegr, Mittelwert 3,86) und Segmentarterien links (ThrSegl, Mittelwert 2,24). 

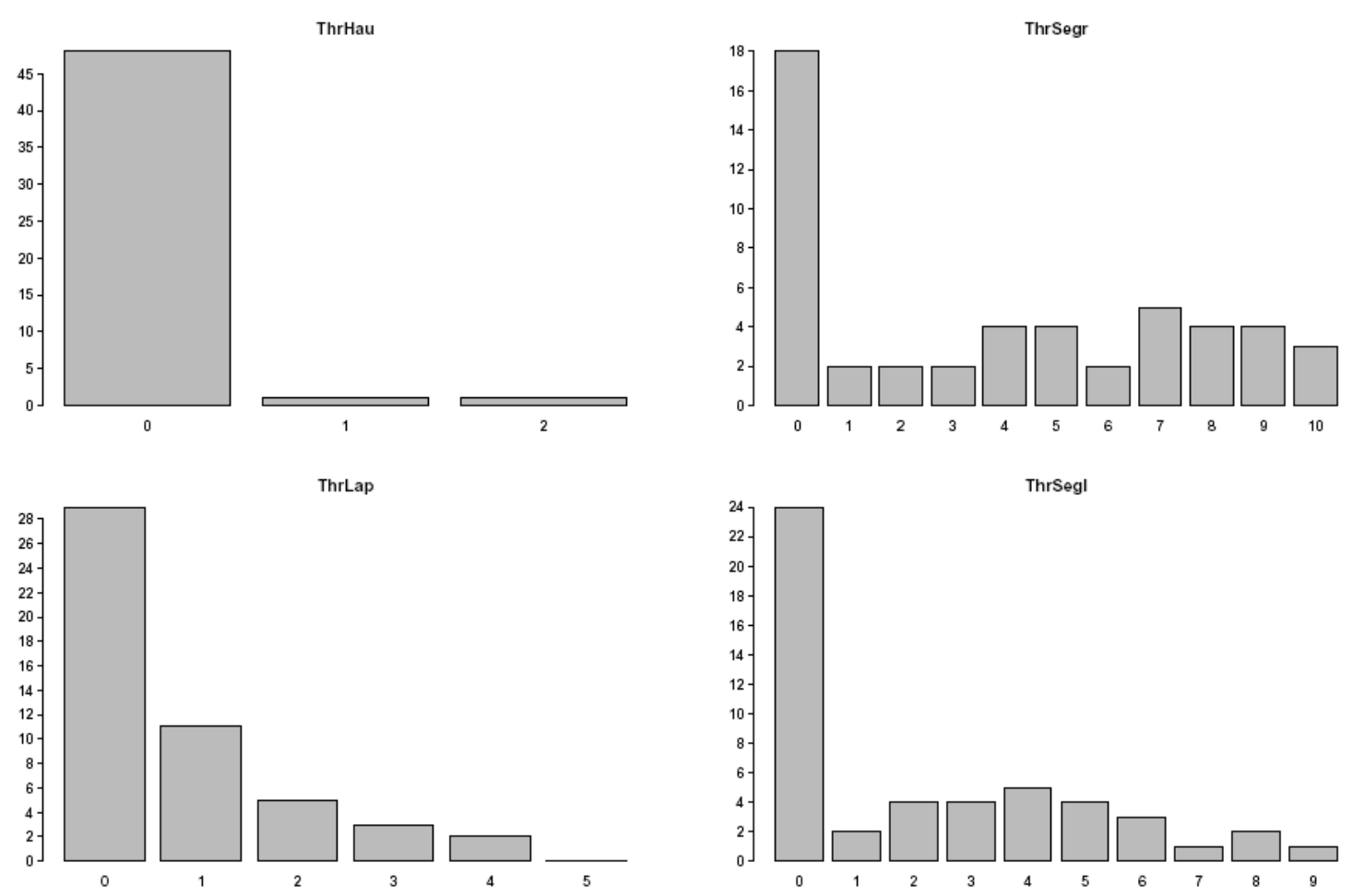

Diag. 7: Lokalisation thrombembolischer Residuen in der Übersichtsangiographie

Der hohe Wert bei Null beinhaltet unter anderem die 13 Patienten, die keine thrombembolisch bedingte pulmonale Hypertonie hatten sowie einen Patienten, der unter einer CTEPH ohne angiographisch sichtbare Residuen litt. Für die hier dargestellten Zahlenverhältnisse der Segmentarterien ergibt sich in der CTEPHGruppe $(n=37)$ eine hochsignifikante Seitendifferenz der Mittelwerte zugunsten der rechten Seite.:

CTEPH: "rechts minus links": Arithmet. Mittel $=2,19$, Median $=2$;

gepaarter, einseitiger t-Test: $\mathrm{p}<0,0001$

$=>$ Mittlere Seitendifferenz "rechts minus links" ist hochsignifikant größer als Null. (95\%-KI für diese Differenz: $[1,54, \infty)$ )

Die Balkendiagramme (Diag. 8) geben an, wie viel Prozent der thrombembolisch veränderten Segmentarterien komplett verschlossen waren. Auf beiden Seiten stellt die Verschlussquote von 0 bis 20 Prozent den größten Anteil (rechts 13, links 9 Patienten). 

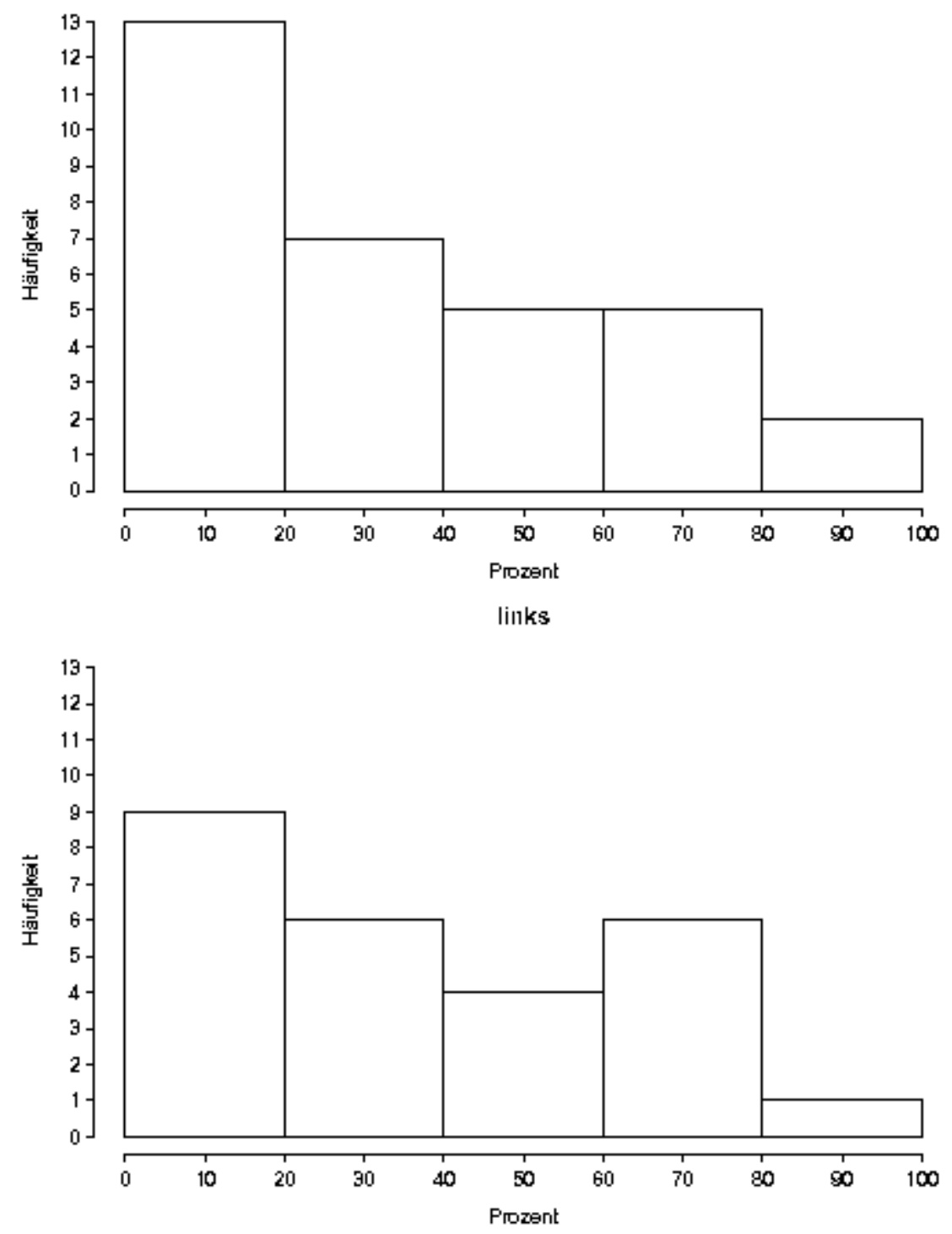

Diag. 8: Anteil verschlossener Segmentarterien links (oben) und rechts

Im Mittel waren rechts 1,4 und links 0,86 Segmentarterien verschlossen. Im t-Test ergibt sich hier für die CTEPH-Patienten kein signifikanter Unterschied zwischen den Seiten $(p=0,4927)$ :

CTEPH: "rechts minus links": Arithmet. Mittel =0,0017, Median = 0,0089; gepaarter, einseitiger t-Test: $p=0,4927$ $=>$ Mittlere Seitendifferenz "rechts minus links" ist nicht signifikant größer als Null (95\%-KI für diese Differenz: $[-0,16, \infty))$. Es ist also keine Seite "bevorzugt".

Wenn thrombembolische Residuen angiographisch vorhanden waren, dann fanden sich meistens in der Lungenperipherie Veränderungen in allen Lappen. Die 13 Patienten mit einer Grunderkrankung verschieden von CTEPH sowie ein Patient mit 
CTEPH ohne angiographisch manifeste Residuen finden sich in der Säule bei Null wieder, ansonsten gab es nur drei CTEPH-Patienten, bei denen weniger als alle Lappen rechts (Diag. 9a)) und sogar nur einen Patienten, bei dem weniger als zwei Lappen links (Diag. 9b)) betroffen waren.
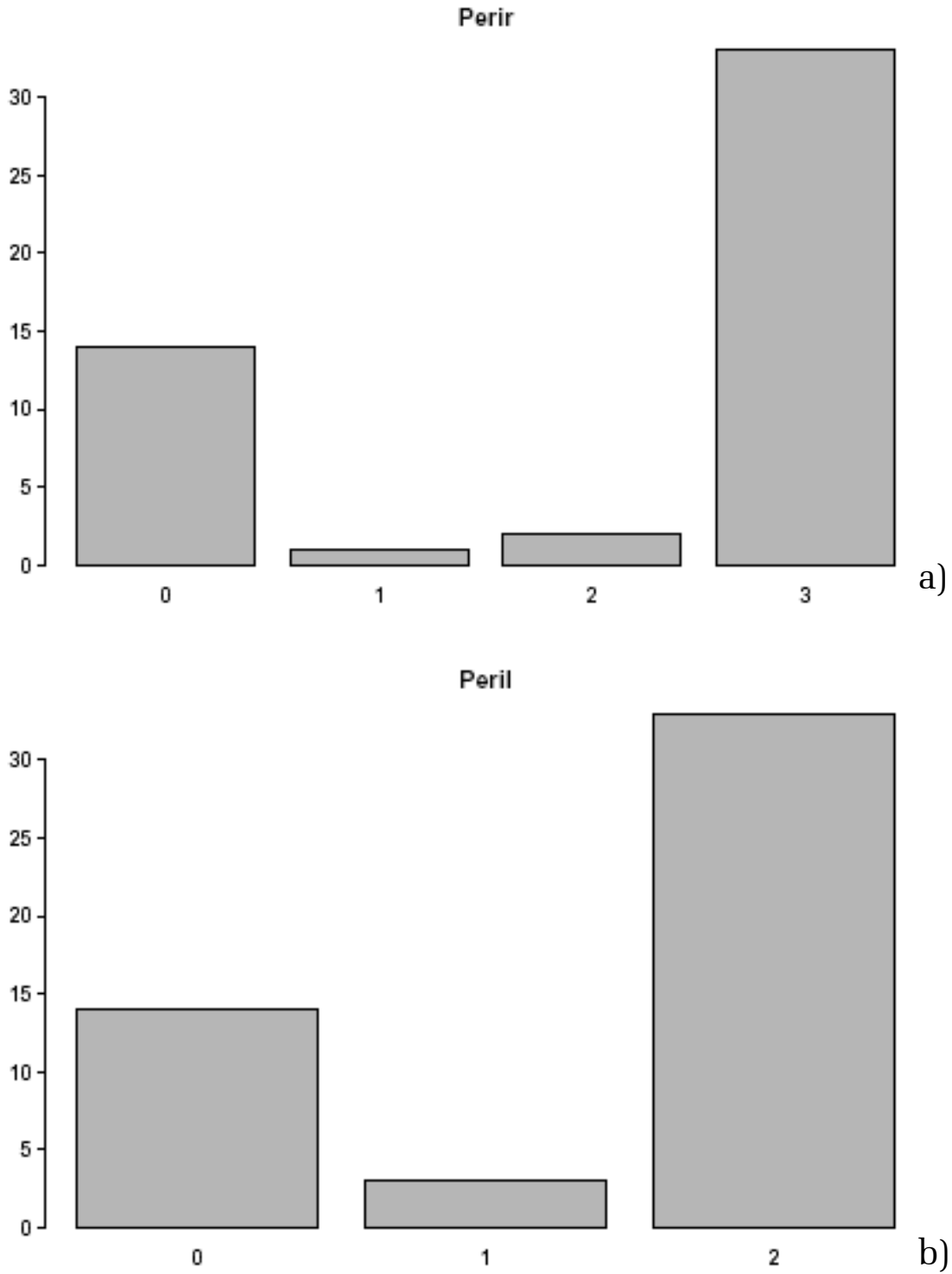

Diag. 9: thrombembolisch befallene periphere Pulmonalarterien 


\subsection{Methodenvergleich}

Im Diagramm 10 sind die Mittelwerte der in Ballon-Okklusionsangiographie und Übersichtsangiographie abzählbaren Aufzweigungsgenerationen gegenübergestellt.

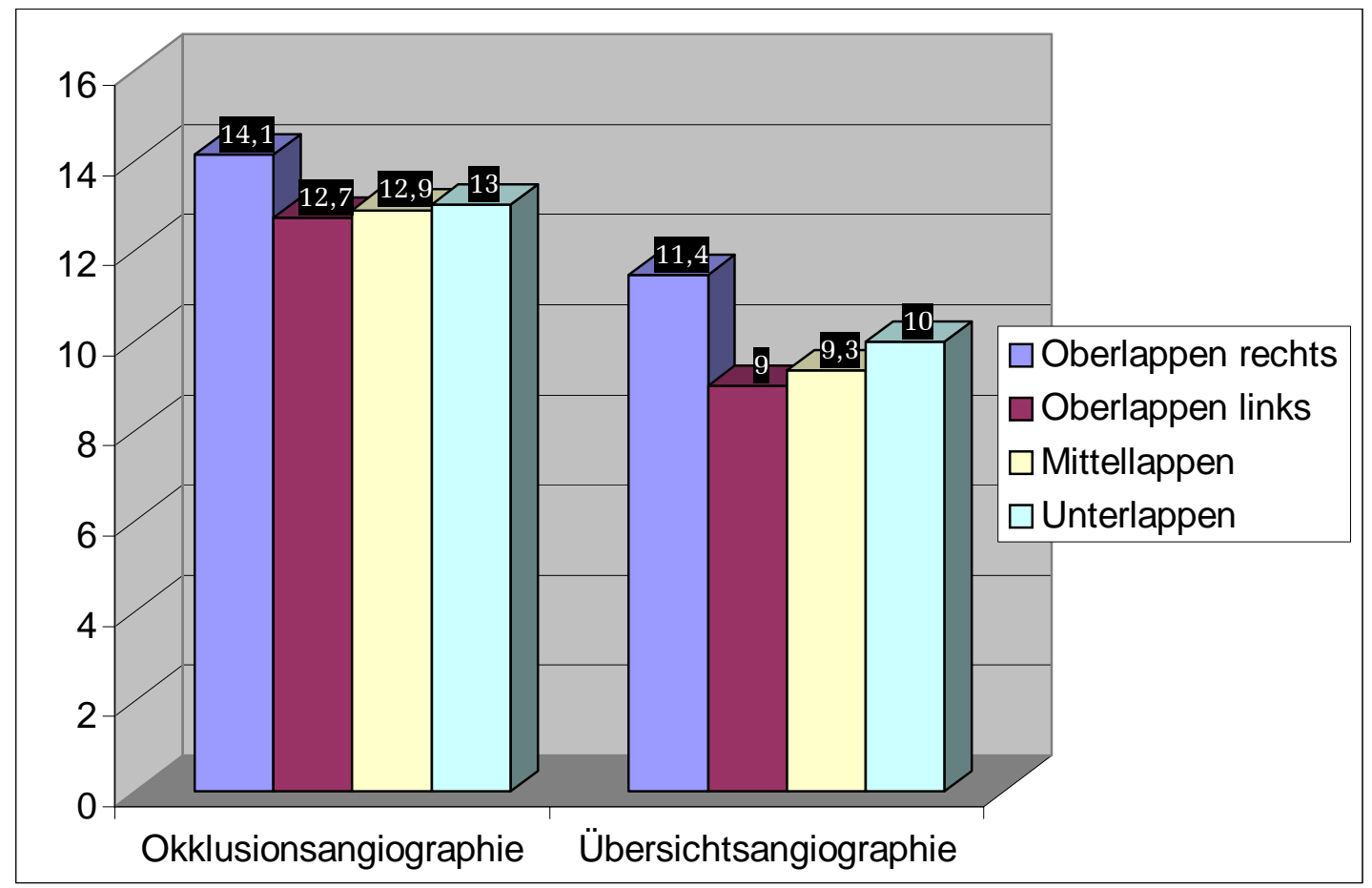

Diag. 10: dargestellte Aufzweigungsgenerationen

Im rechten Oberlappen bildete die Okklusionsangiographie (im Folgenden kurz OA) im Mittel 2,7 Generationen mehr ab. Sie war der Übersichtsangiographie (im Folgenden kurz ÜA) damit signifikant überlegen (gepaarter t-Test: $p<0,0001)$. Im linken Oberlappen wurde nur in sieben Fällen eine Okklusionsangiographie durchgeführt. Der Unterschied zwischen den beiden Verfahren lag im Mittel bei 3,7 Generationen. Der Unterschied erweist sich im gepaarten t-Test als signifikant $(p=0,008)$. Im Mittellappen wurden nur neun Vergleichsmessungen durchgeführt, der mittlere Unterschied lag hier bei 3,6 Verzweigungen zugunsten der BallonOkklusionsangiographie. Diese Überlegenheit in der Detailgenauigkeit war statistisch signifikant (gepaarter t-Test: $p=0,0004$ ). In der Grafik und in den Berechnungen wurden alle Messungen in beiden Unterlappen zusammengefasst. Der Mittelwert der abzählbaren Aufzweigungsgenerationen lag für die BallonOkklusionsangiographie bei 13, für die Übersichtsangiographie bei 10,0 (Differenz der Mittelwerte 3,00). Das bedeutet, dass auch in den Unterlappen die Wahl des 
Angiographieverfahrens einen hochsignifikanten Einfluss auf die Detailgenauigkeit hat (hochsignifikanter Einfluss des Angiographietyps in einem "Generalized Linear Mixed Model" mit Poisson-Link für die Zahl der Aufzweigungsgenerationen bei wiederholten Beobachtungen pro Individuum: $p<0,0001$ ).

Das Diagramm 11 illustriert die relativen Häufigkeiten, mit denen die Ballon-Okklusionsangiographie mehr Gefäße darstellte als die Übersichtsangiographie. Es zeigt sich, dass auf Höhe der Segmentarterien die Übersichtsangiographie ausreicht, um alle Gefäße zu erfassen. In 30\% der Fälle zeigte die Ballon-Okklusionsangiographie mehr Subsegmentarterien als die Übersichtsangiographie (einseitiges, exaktes 95\%-Binomial-Konfidenzintervall für die Häufigkeit der Überlegenheit der OA gegenüber der ÜA (im Folgenden nur kurz "95\%-Bin.-KI"): [19\%, 100\%)). Auch der mittlere Unterschied in der gezeigten Anzahl der Gefäße von 0,62 (95\%Konfidenzintervall der Differenz (i. F. kurz "95\%-KI") [0,34, $\infty)$ ) ist signifikant (gepaarter, einseitiger t-Test: $\mathrm{p}=0,0002$ ). Die Erfolgswahrscheinlichkeit steigt in der nächsten Generation, den Gefäßen 6. Ordnung, sogar auf 64\% (95\%-Bin.-KI: [51\%, 100\%). Der mittlere Unterschied zwischen OA und ÜA ist 2,4 (95\%-KI: $[0,34, \infty))$ und damit signifikant größer als Null $(\mathrm{p}<0,0001)$.

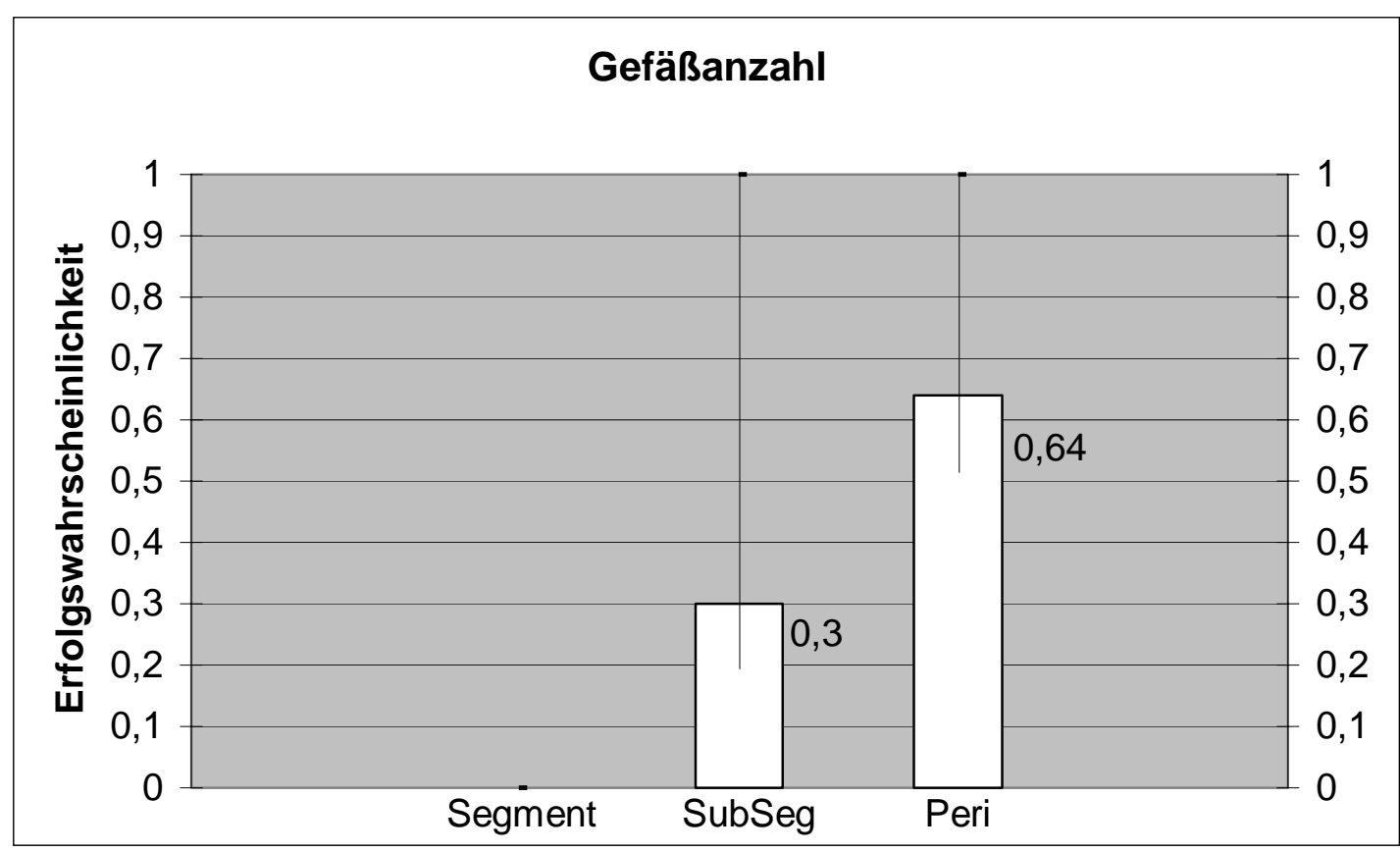

Diag. 11: dargestellte Gefäße im Methodenvergleich (Erfolgswahrscheinlichkeit als Säule, 95\%-Konfidenzintervall als vertikaler Strich)

Die Ballon-Okklusionsangiographie ist also distal der Segmentarterien signifikant besser in der Darstellung der Gefäßverzweigungen als die Übersichtsangiographie. 
Auf ein Auszählen der noch weiter peripher gelegenen Gefäße wurde aus Praktikabilitätsgründen verzichtet (daher fehlt eine entsprechende Säule für die Peripherie im Diagramm).

In der Übersichtsangiographie waren alle Segmentarterienstenosen wiederzufinden, die in der Ballon-Okklusionsangiographie dargestellt wurden (Diag. 12). Bereits auf Höhe der Subsegmentarterien zeigte die Ballon-Okklusionsangiographie mit 22\%iger Häufigkeit mehr Stenosen als das Standardverfahren (95\%-Bin.-KI: $[12 \%, 100 \%)$ ); der Unterschied in der Anzahl der Stenosen von 0,28 (95\%-KI: [0,12, $\infty)$ ) ist signifikant größer Null (gepaarter, einseitiger t-Test: $p=0,0016$ ). Die Erfolgswahrscheinlichkeit nimmt in den Gefäßen sechster Ordnung auf 38\% (95\%Bin.-KI: [26\%, 100\%); der Unterschied in der Stenosenzahl: 0,82 mit 95\%-KI [0,47, $\infty$ ) ist signifikant größer Null im gepaarten, einseitigen t-Test: $\mathrm{p}<0,0001)$ und in der Peripherie auf 56\% (95\%-Bin.-KI: [43\%, 100\%)) zu.

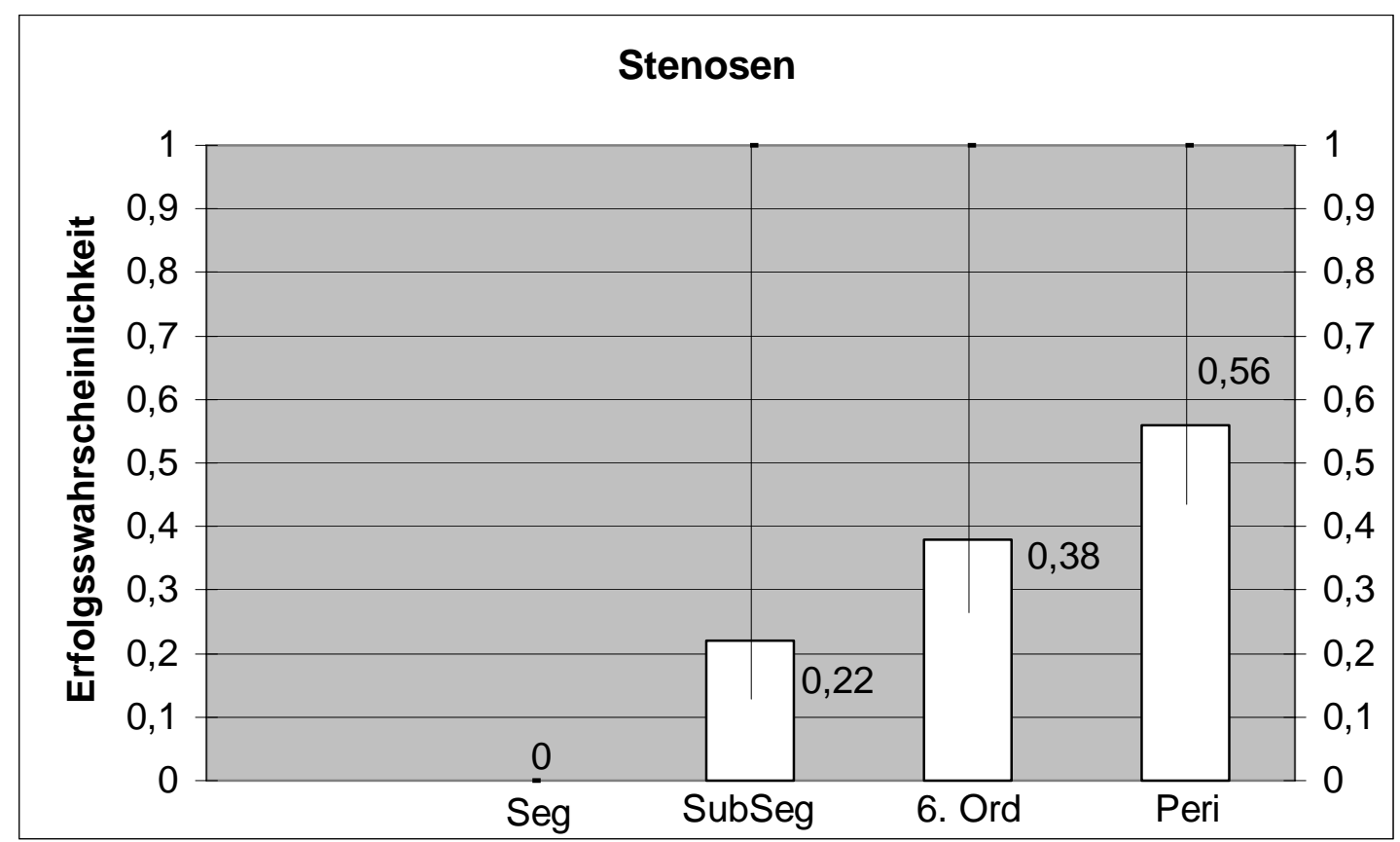

Diag. 12: abgrenzbare Stenosen im Methodenvergleich

Untersucht man nur die Patienten mit einer CTEPH, so ist die Ballon-Okklusionsangiographie distal der Segmentarterien signifikant besser als die Übersichtsangiographie im Nachweis von Stenosen. Diese Vorgehensweise erscheint legitim, da Stenosen als thrombembolisches Residuum nahezu ausschließlich bei Patienten mit CTEPH auftreten. Bei den übrigen Patienten, die keine Stenosen haben, kann 
der Vergleich der beiden Untersuchungsverfahren keinen Unterschied zutage fördern.

\section{Nur CTEPH:}

SubSeg:

relative OA-Überlegenheitshäufigkeit (im Folgenden kurz "rel HK") = 30 \% (95\%-Bin.-KI: [17\%, 100\%));

mittlere Stenosenzahldifferenz $=0,38(95 \%-K I:[0,17, \infty))$, gepaarter, einseitiger t-Test: $\mathrm{p}=0,0015$.

6. Ord.:

rel HK = 49 \% (95\%-Bin.-KI: [34\%, 100\%));

mittlere Stenosenzahldifferenz $=1,1$ (95\%-KI: [0,64, $\infty)$ ), gepaarter, einseitiger t-Test: $\mathrm{p}<0,0001$.

Peri:

rel HK = 70,3 \% (95\%-Bin.-KI: [55\%, 100\%))

Strickleitersysteme (Webs und Bands) sind ebenfalls typische thrombembolische Residuen. Die Häufigkeiten, mit denen die Ballon-Okklusionsangiographie mehr Webs und Bands nachweist, sind im Diagramm 13 für das Gesamtkollektiv aufgetragen. Hier zeigt sich im Vergleich mit den vorangegangenen Grafiken, dass erstmals bereits auf Höhe der Segmentarterien die Ballon-Okklusionsangiographie in 11\% der Fälle besser ist (95\%-Bin.-KI: [3,7\%, 100\%). Der Unterschied in der Merkmalszahl: 0,11 mit 95\%-KI [0,02, $)$ ist signifikant größer Null im gepaarten, einseitigen t-Test: $p=0,02)$. Die Häufigkeiten nehmen zur Peripherie hin zu auf 46\% (95\%-Bin.-KI: [33\%, 100\%); die Merkmalszahldifferenz: 0,95 mit 95\%-KI $[0,66, \infty)$ ist signifikant größer Null im gepaarten, einseitigen t-Test: $p<0,0001)$ in den Subsegmentarterien, 62\% (95\%-Bin.-KI: [49\%, 100\%); die Merkmalszahldifferenz: 2,24 mit 95\%-KI [1,6, $\infty)$ ist signifikant größer Null im gepaarten, einseitigen t-Test: $\mathrm{p}<0.0001$ ) in den Arterien 6. Ordnung und 58\% (95\%-Bin.-KI: [45\%, 100\%)] in der Peripherie zu. 


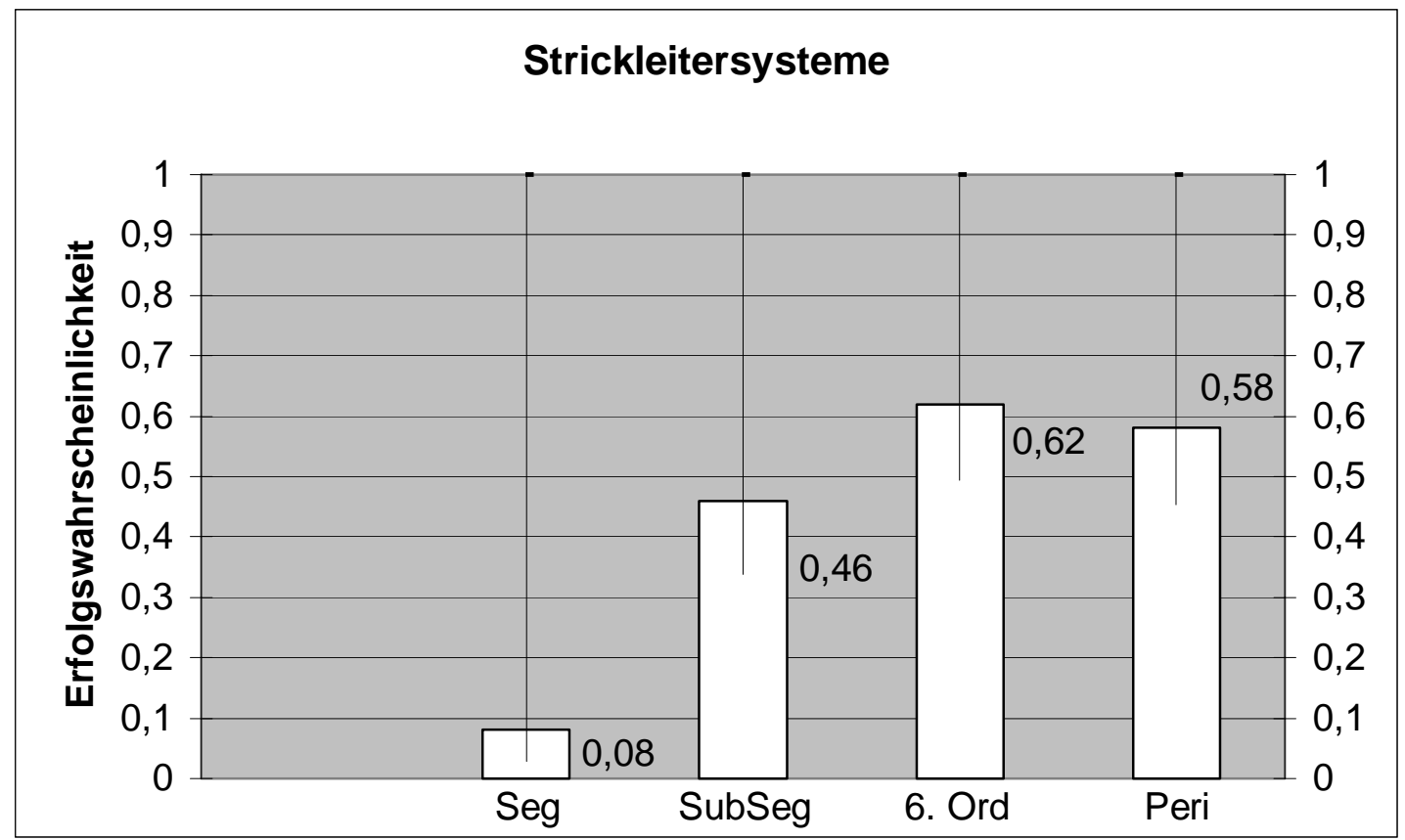

Diag. 13: abgrenzbare Strickleitersysteme (Webs und Bands) im Methodenvergleich

Die isolierte Betrachtung der Patienten mit CTEPH ergibt eine signifikante Überlegenheit des Verfahrens:

Nur CTEPH:

Seg:

rel HK = 15,4\% (95\%-Bin.-KI: [5,4\%, 100\%));

mittl. Merkmalszahldiff. $=0,15$ (95\%-KI: $[0,03, \infty))$, gepaarter, einseitiger t-Test: $p=0,02$

SubSeg:

rel HK = 59 \% (95\%-Bin.-KI: [44\%, 100\%));

mittl. Merkmalszahldiff. = 1,3 (95\%-KI: [0,9, $\infty)$ ), gepaarter, einseitiger t-Test: $\mathrm{p}<0,0001$.

6. Ord:

rel HK = 84 \% (95\%-Bin.-KI: [70\%, 100\%));

mittl. Merkmalszahldiff. = 3,0 (95\%-KI: [2,3, $\infty)$ ), gepaarter, einseitiger t-Test: $\mathrm{p}<0,0001$.

Peri:

rel HK = 75,7\% (95\%-Bin.-KI: [61\%, 100\%)) 
Gefäßverschlüsse sind in den zentralen Gefäßen nahezu spezifisch für Lungenembolien. In der Peripherie kommen sie auch bei pulmonaler Hypertonie anderer Genese, so z.B. bei primärer pulmonaler Hypertonie, vor.

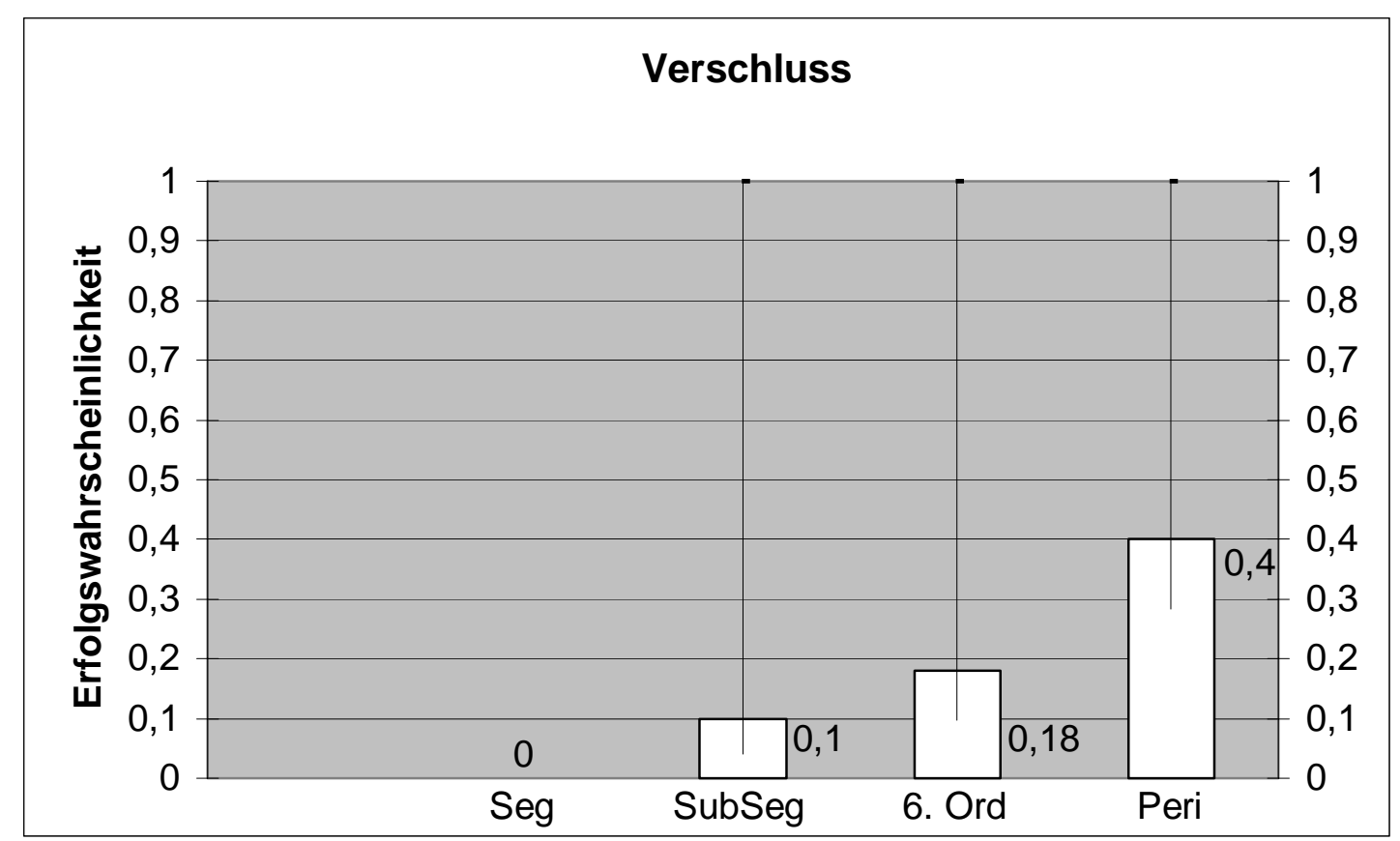

Diag. 14: Gefäßverschlüsse im Methodenvergleich

Die Untersuchungsverfahren sind im Nachweis von Segmentarterienverschlüssen gleichwertig. Im Diagramm 14 steigen die Erfolgswahrscheinlichkeiten der BallonOkklusionsangiographie zur Peripherie hin kontinuierlich an: Subsegmentarterien 10\% (95\%-Bin.-KI: [4\%, 100\%); die Verschlussanzahldifferenz: 0,1 mit 95\%-KI $[0,02, \infty)$ ist signifikant größer Null im gepaarten, einseitigen t-Test: $p=0,012)$, in Arterien 6. Ordnung 18\% (95\%-Bin.-KI: [9\%, 100\%); die Verschlussanzahldifferenz: 0,2 mit 95\%-KI $[0,09, \infty)$ ist signifikant größer Null im gepaarten, einseitigen t-Test: $\mathrm{p}=0,0015)$, peripher 40\% (95\%-Bin.-KI: [28\%, 100\%).

In der CTEPH-Gruppe ist die Ballon-Okklusionsangiographie bereits ab dem Niveau der Subsegmentarterien signifikant überlegen:

Nur CTEPH:

SubSeg:

rel HK = $11 \%$ (95\%-Bin.-KI: [3\%, 100\%));

mittl. Verschlussanzahldiff. $=0,11(95 \%-\mathrm{KI}:[0,02, \infty))$, gepaarter, einseitiger t-Test: $\mathrm{p}=0,022$.

6. Ord:

rel HK = $24 \%$ (95\%-Bin.-KI: [13\%, 100\%)); 
mittl. Verschlussanzahldiff. $=0,27$ (95\%-KI: [0,12, $\infty)$ ), gepaarter, einseitiger t-Test: $\mathrm{p}=0,0013$.

Peri:

rel HK $=51,4 \%$ (95\%-Bin.-KI: [36\%, 100\%))

Die Ballon-Okklusionsangiographie macht thrombembolische Residuen in den kleinen Pulmonalarterien bis in die kleinsten Details sichtbar. Abbildung 12a zeigt eine kurze membranartige Stenose (schwarzer Pfeil) und eine nahezu verschlossene Subsegmentarterie (gelber Pfeil). In Abbildung 12b finden sich zarte Strickleitersysteme in einer Bifurkation (schwarzer Pfeil) und ein verschlossener Subsegmentast (gelber Pfeil) mit nachfolgender Rarefikation der präkapillären Arterien. Abbildung 12c zeigt frischere Embolien im Subsegmentbereich des linken Unterlappens.
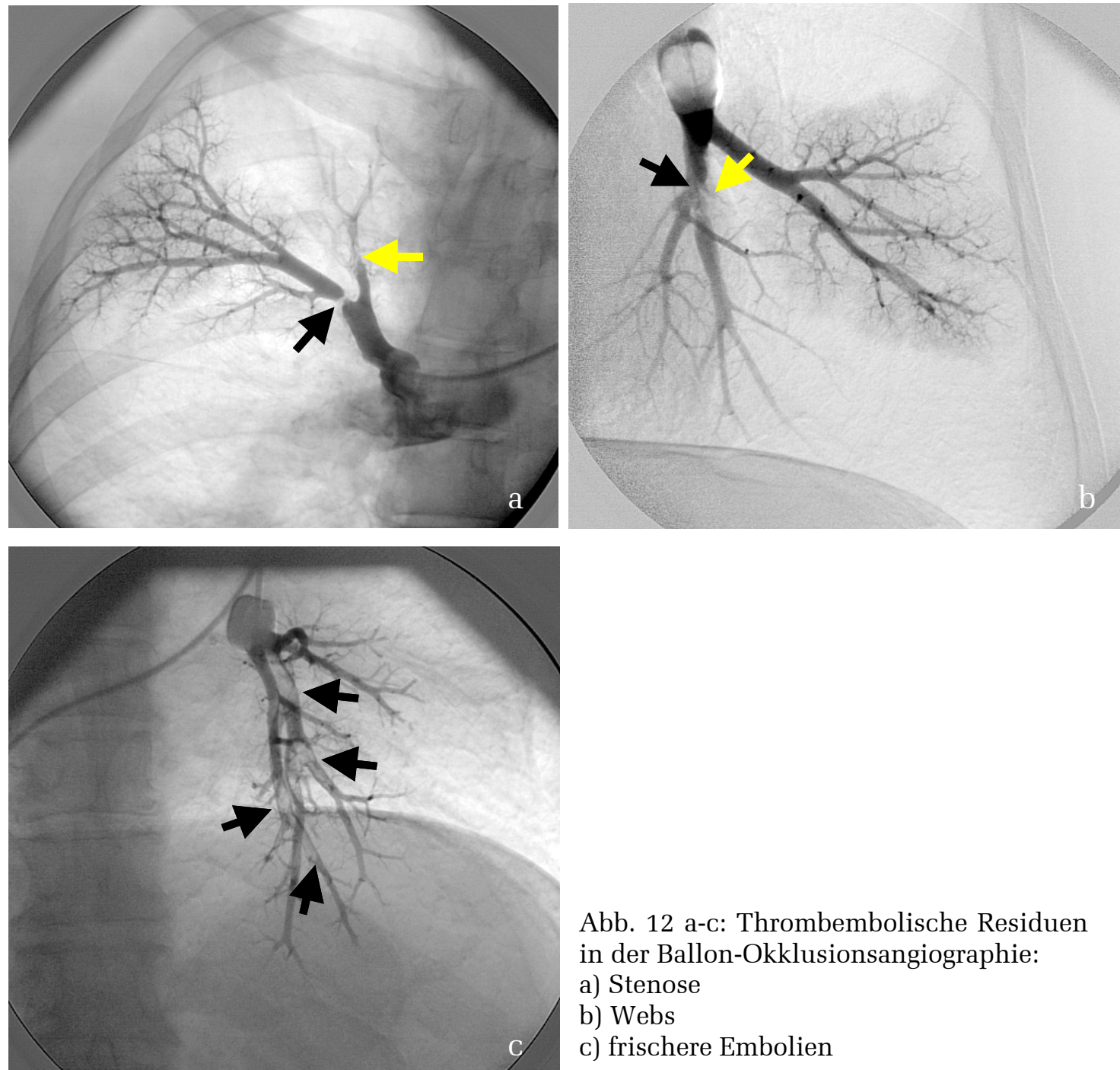

Abb. 12 a-c: Thrombembolische Residuen in der Ballon-Okklusionsangiographie:

a) Stenose

b) Webs

c) frischere Embolien 
Die Unterschiede im Nachweis von Kalibersprüngen (Diagramm 15) sind gering im Segment- (0\%) und Subsegmentarterienniveau (10\%, 95\%-Bin.-KI: [4\%, 100\%); die Merkmalszahldifferenz: 0,1 mit 95\%-KI [0,02, $\infty$ ) ist signifikant größer Null im gepaarten, einseitigen t-Test: $p=0,01)$. In den Gefäßen 6. Ordnung steigt die Häufigkeit auf 20\% (95\%-Bin.-KI: [11\%, 100\%); die Merkmalszahldifferenz: 0,38 mit 95\%-KI $[0,15, \infty)$ ist signifikant. größer Null im gepaarten, einseitigen t-Test: $\mathrm{p}=0,003$ ). Eine deutliche Überlegenheit der Ballon-Okklusionsangiographie ist in den peripheren Gefäßen abzulesen, das Verfahren zeigt hier in 60\% (95\%-Bin.-KI: [47\%, 100\%)) der Fälle mehr periphere Kalibersprünge als die Übersichtsangiographie.

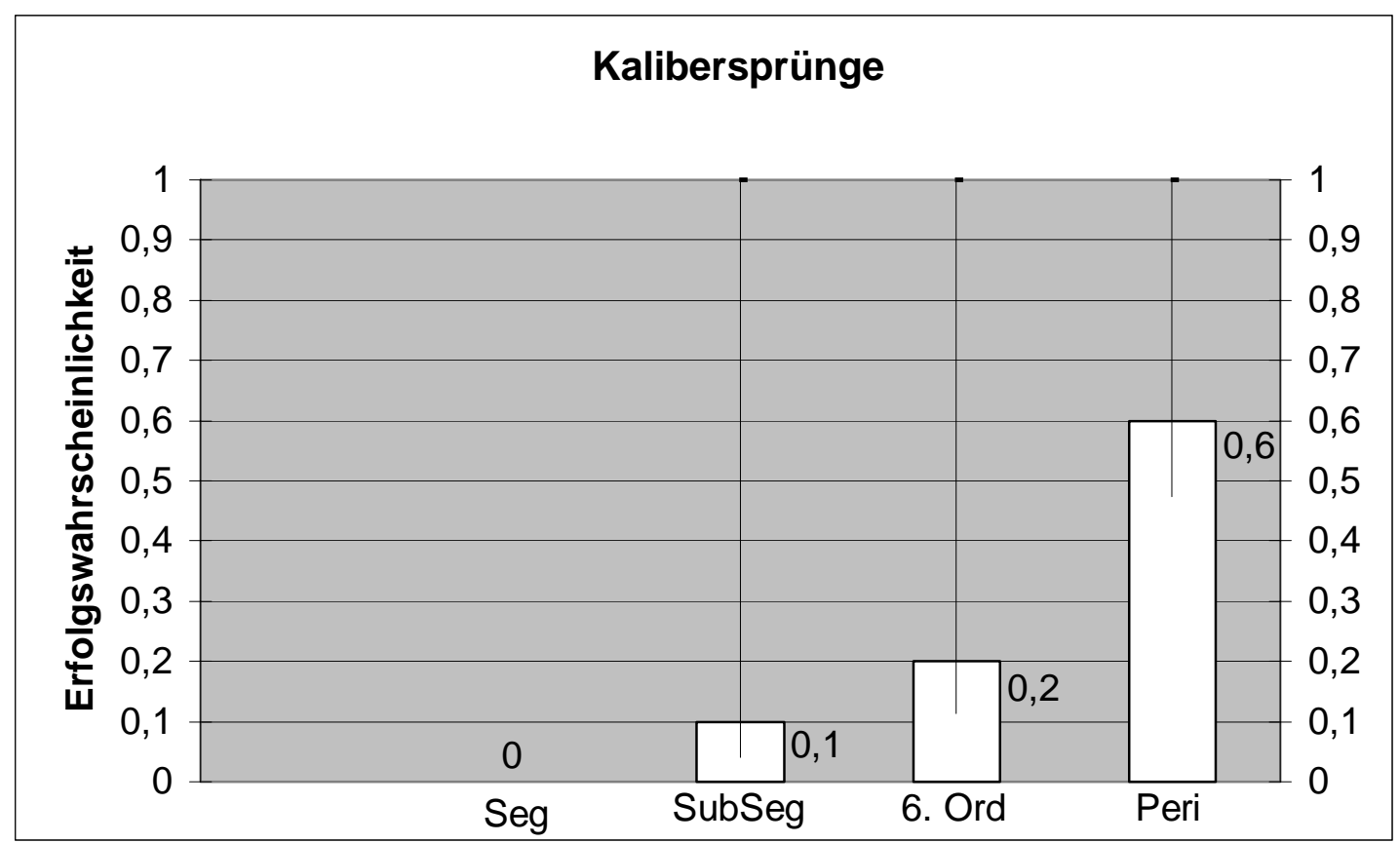

Diag. 15: Kalibersprünge im Methodenvergleich

Kalibersprünge sind ein unspezifisches angiographisches Merkmal der chronischen pulmonalen Hypertonie. Eine isolierte Betrachtung der CTEPH-Patienten ist daher nicht sinnvoll.

Anastomosen zwischen durchflossenen und benachbarten, zentral verschlossenen Pulmonalarterien sowie zwischen Pulmonalarterien und subpleuralen Bronchialarterien kommen im Segmentarterienniveau nicht vor. Im Subsegmentarterienniveau zeigte sich, dass in $4 \%$ der Fälle die Okklusionsangiographie mehr Kollateralen erfasst als die Übersichtsangiographie (95\%-Bin.-KI: [0,7\%, 100\%); die 
Anastomosenzahldiff.: 0,04 mit 95\%-KI [-0,007, $)$ ist nicht signifikant größer Null im gepaarten, einseitigen t-Test: $\mathrm{p}=0,08)$. Die Wahrscheinlichkeit nimmt auf Höhe der Gefäße sechster Ordnung auf 6\% (95\%-Bin.-KI: [1,6\%, 100\%); die Anastomosenzahldifferenz: 0,06 mit 95\%-KI $[0,003, \infty)$ ist nur leicht signifikant größer Null im gepaarten, einseitigen t-Test: $p=0,04)$ und in der Peripherie sprunghaft auf 40\% (95\%-Bin.-KI: [28\%, 100\%)) zu (Diag. 16). Diese Zahlen beziehen sich auf das ausgewertete Studienkollektiv. Die Analyse der Rohdaten zeigt, dass Anastomosen im Subsegmentarterienniveau nur zweimal und im Niveau der Gefäße sechster Ordnung nur dreimal aufgetreten waren. In allen fünf Fällen konnten diese Anastomosen in der Übersichtsangiographie nicht wiedergefunden werden. Die geringe Erfolgswahrscheinlichkeit in diesen Gefäßgenerationen ergibt sich also aus der großen Anzahl der Fälle, in denen überhaupt keine Anastomosen sichtbar sind und somit auch keine Unterschiede zwischen Okklusionsangiographie und Übersichtsangiographie bestehen.

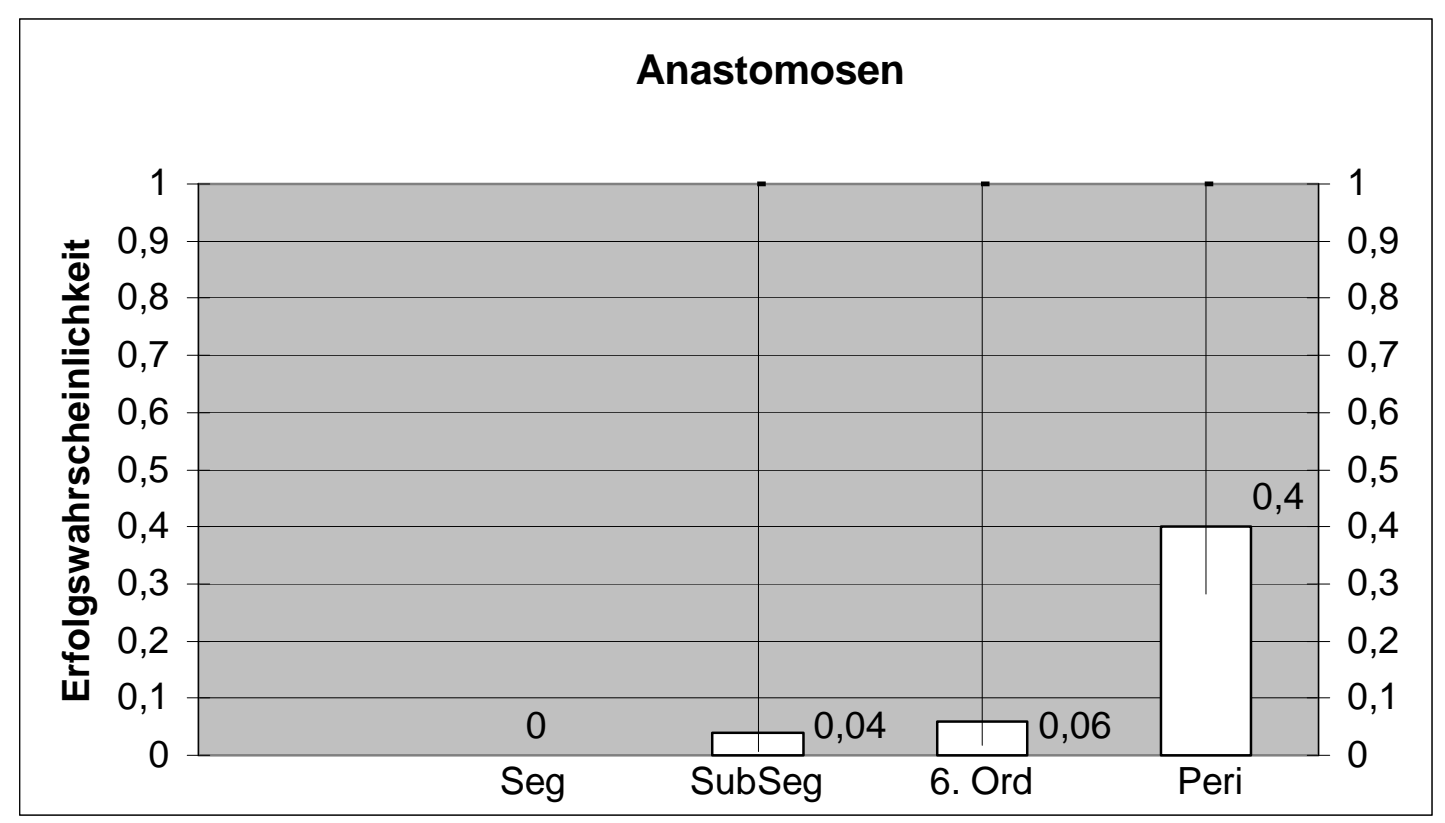

Diag. 16: Anastomosen im Methodenvergleich

Diejenigen Anastomosen, die zu einer peripheren Wiederauffüllung zentral verschlossener Pulmonalarterien führen, finden sich ausschließlich bei Patienten mit CTEPH. Sie sind somit spezifisch für diese Diagnose. Von 36 Patienten mit CTEPH zeigten 17 eine Kollateralisation proximal verschlossener Gefäße in der Ballon-Okklusionsangiographie (ca. 47\%). Drei Patienten hatten sowohl Anastomosen mit Wiederauffüllung benachbarter Pulmonalarterien als auch solche mit Kontras- 
tierung subpleuraler Bronchialarterien. Bei fünf Patienten mit CTEPH war nur letztgenannter Anastomosentyp zu finden. Die Kontrastierung subpleuraler Bronchialarterienäste aus dem pulmonalarteriellen System jedoch lässt sich nicht einer Diagnosegruppe zuordnen und ist somit bezüglich der Differentialdiagnose unspezifisch.

Abbildung 13 zeigt die Spätfolgen einer schweren Thrombembolie: Die Originalverzweigungen der rechten Unterlappenarterie sind verschlossen und ersetzt durch einen dichten Plexus aus geschlängelten Bronchialarterien.
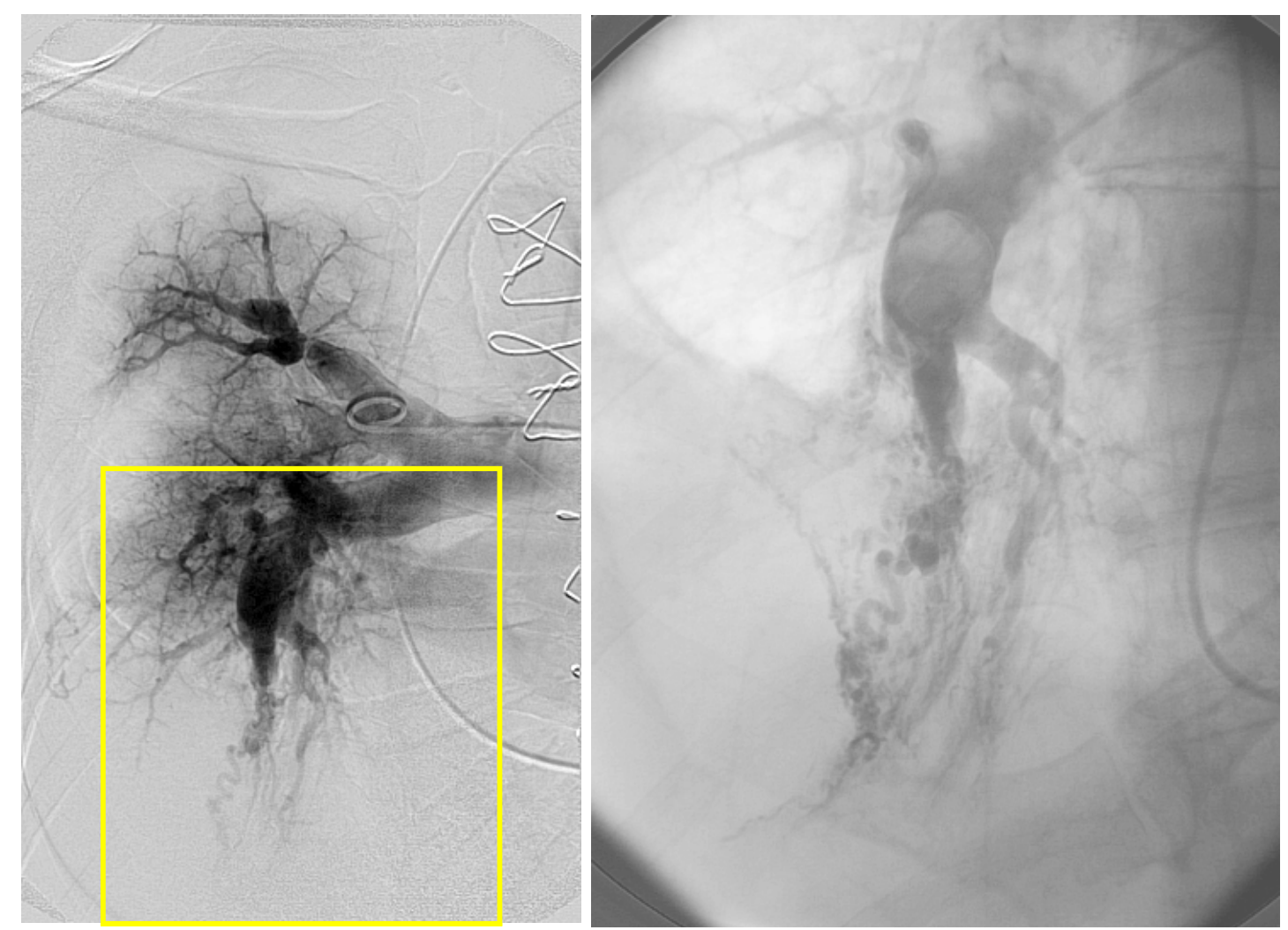

Abb. 13: Spätfolgen einer schweren Thrombembolie im rechten Unterlappen in der Übersichtsangiographie (a) und in der Ballon-Okklusionsangiographie (b) 
In Abbildung 14 ist die Ballon-Okklusionsangiographie einer Segmentarterie im linken Unterlappen gezeigt. Man erkennt den stummelförmigen Eingang in eine verschlossene Subsegmentarterie sowie mehrere Stenosen, die durch thrombembolische Residuen hervorgerufen werden. Eine kleine subpleurale Arterie formt eine Anastomose mit einer anderen, zentral verschlossenen Pulmonalarterie. Diese Kollateralisation eines Pulmonalarterienverschlusses war in der Übersichtsangiographie (nicht abgebildet) nicht erkennbar.

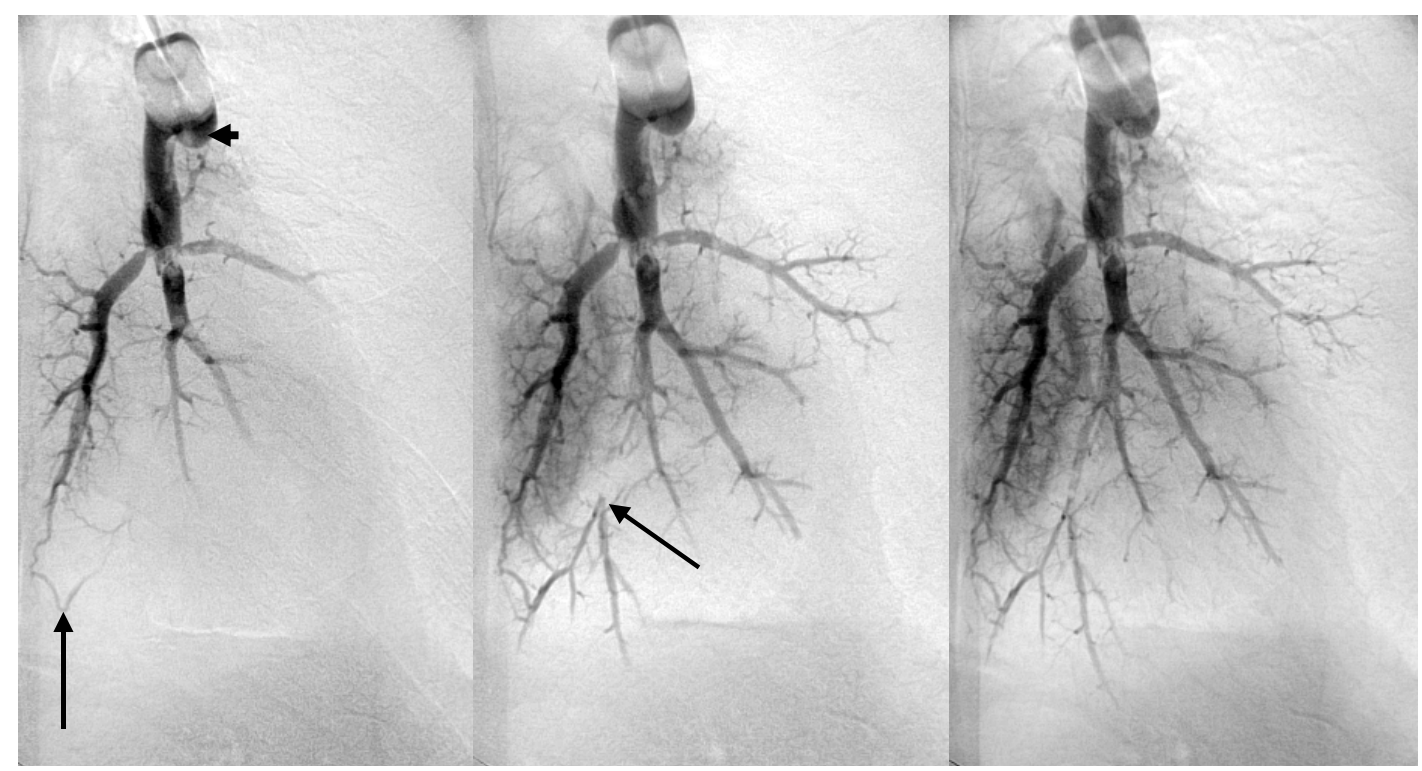

Abb. 14: Ballon-Okklusionsangiographie im linken Unterlappen mit Verschluss einer Subsegmentarterie (Pfeilspitze), Stenosen und kollateralisiertem peripherem Verschluss (Pfeile)

Abbildung 15 zeigt einen noch ausgeprägteren Fall. Die Anastomose mit einer zentral verschlossenen Pulmonalarterie erscheint wie ein angiomartiges Gebilde. Möglicherweise formen hypertrophierte Bronchialarterien den verbindenden Schenkel zwischen durchbluteten und verschlossenen Pulmonalarterien.

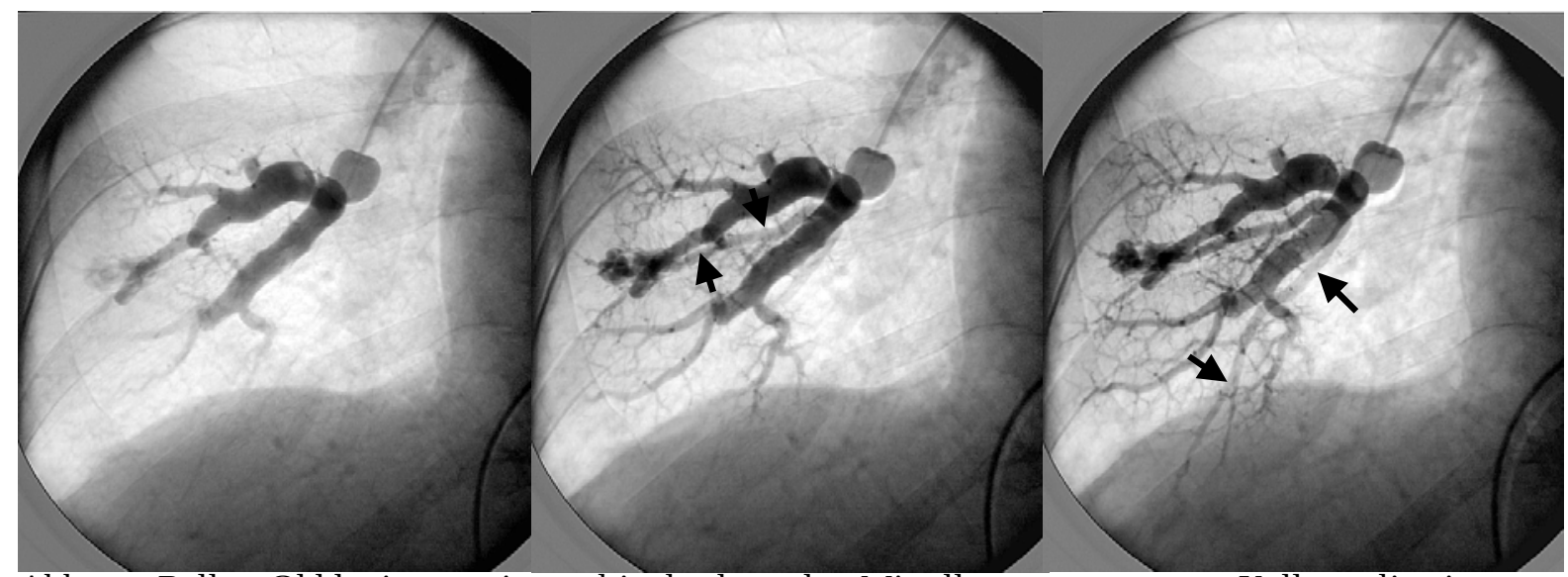

Abb. 15: Ballon-Okklusionsangiographie des lateralen Mittellappensegmentes. Kollateralisation eines Subsegmentverschlusses über ein peripheres, angiomartiges Gefäßknäuel. 
Patienten, die an einer CTEPH litten und kollateralisierte Verschlüsse in der Ballon-Okklusionsangiographie zeigten, hatten durchschnittlich einen pulmonalarteriellen Mitteldruck von 52,3 mmHg (Diagramm 17). Die Patienten ohne angiographisch nachweisbare Kollateralen hatten im Mittel einen niedrigeren Druck (45 mmHg). Dieser Unterschied erweist sich statistisch als leicht signifikant (Zweistichproben- t-Test: $\mathrm{p}=0,087$ ).

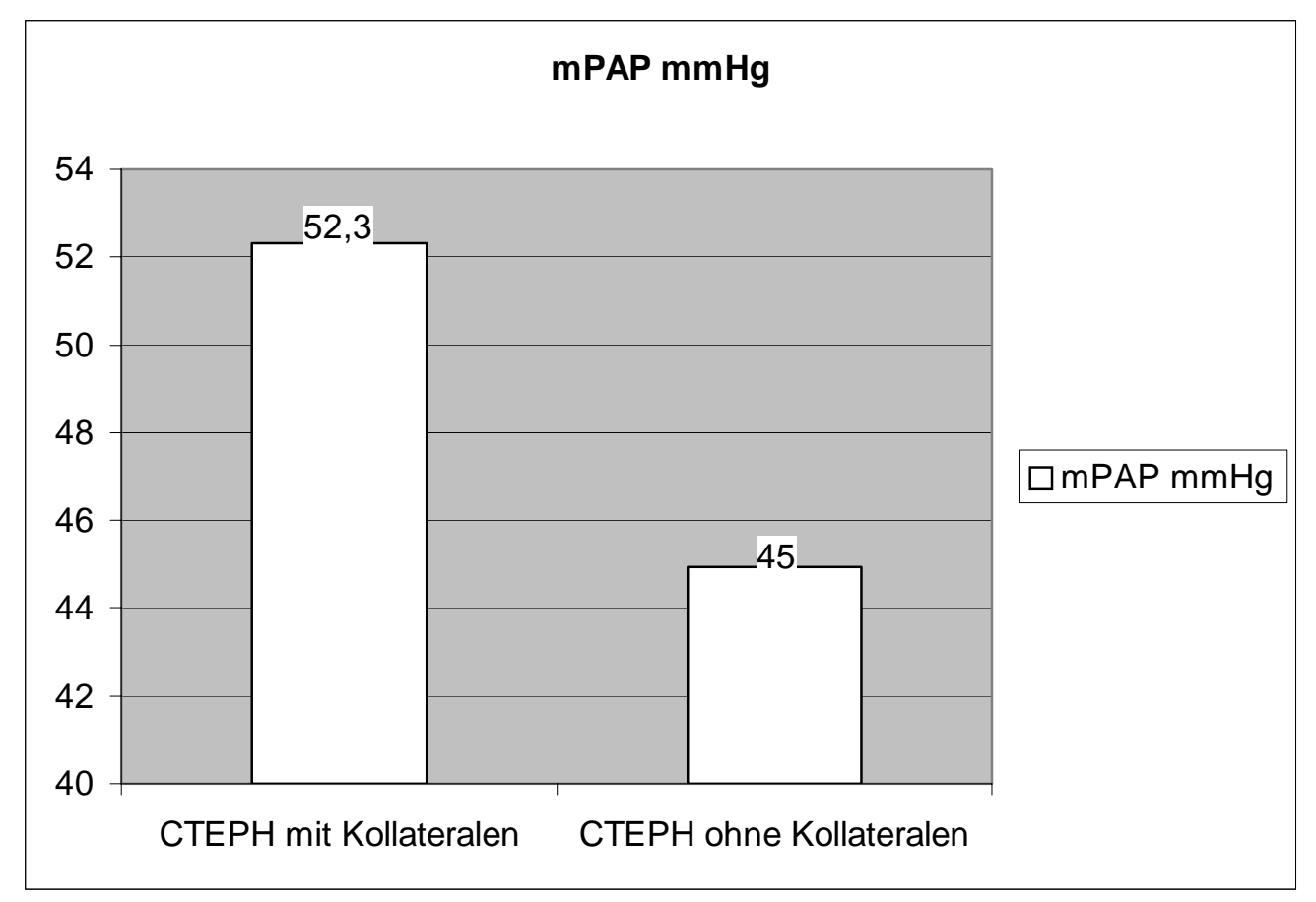

Diag. 17: pulmonalarterieller Mitteldruck bei CTEPH mit und ohne Kollateralen 
In dem Dotchart (Grafik 8, vgl. 2.6.1) für den Nachweis von Webs und Bands in den Gefäßen sechster Ordnung sind die Ergebnisse des Vergleiches zwischen BallonOkklusionsangiographie (o) und Übersichtsangiographie (+) gegeneinander aufgetragen. Hier wird beispielhaft demonstriert, dass mit zunehmender Häufigkeit des Merkmales in der Ballon-Okklusionsangiographie auch der Unterschied zwischen den beiden Verfahren größer wird. In keinem Fall konnte die Übersicht (+) mehr Webs und Bands nachweisen als die Ballon-Okklusionsangiographie.

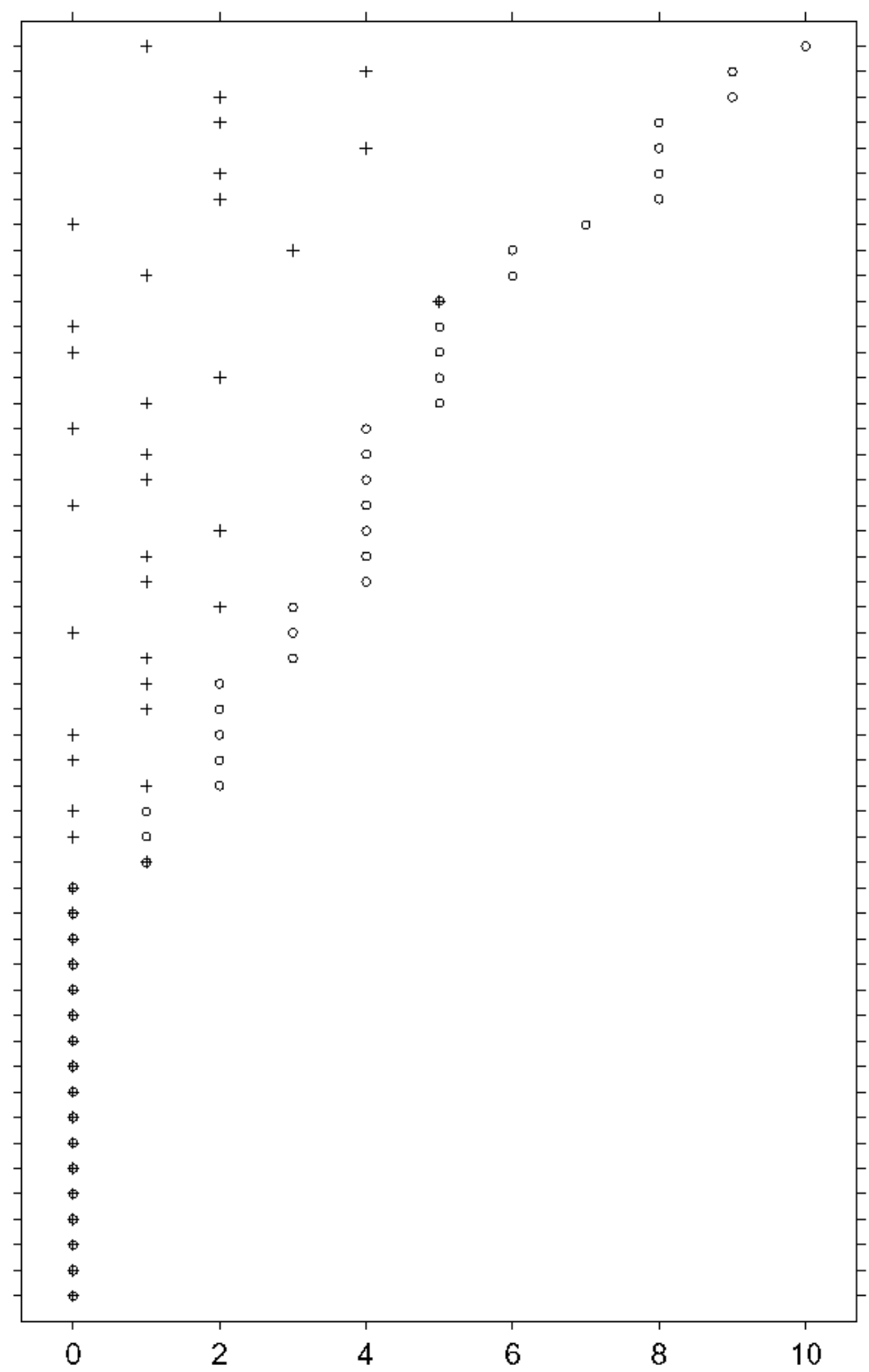

Grafik 8: Häufigkeitsverteilung von Strickleitersystemen in Übersichtsangiographie und Ballon-Okklusionsangiographie in den Gefäßen 6. Ordnung 
Solche Dotcharts (vgl. 2.6.1) sind auch für folgende Merkmale erstellt worden: Zahl der abgrenzbaren Gefäße, Stenosen, Verschlüsse, Kalibersprünge und Anastomosen.

Folgender Dotchart (Grafik 9) fasst alle thrombembolischen Residuen (Webs und Bands, Stenosen, Verschlüsse) in allen Gefäßabschnitten (Segment, Subsegment, 6. Ordnung, Peripherie) zusammen. Auch hier entspricht jeweils ein Symbolpaar in der Horizontalen einem Patienten. In keinem Fall steht das Symbol + rechts vom Symbol o, d.h. die Übersichtsangiographie erkennt maximal genauso viele thrombembolische Residuen wie die Ballon-Okklusionsangiographie, häufig jedoch zum Teil deutlich weniger.

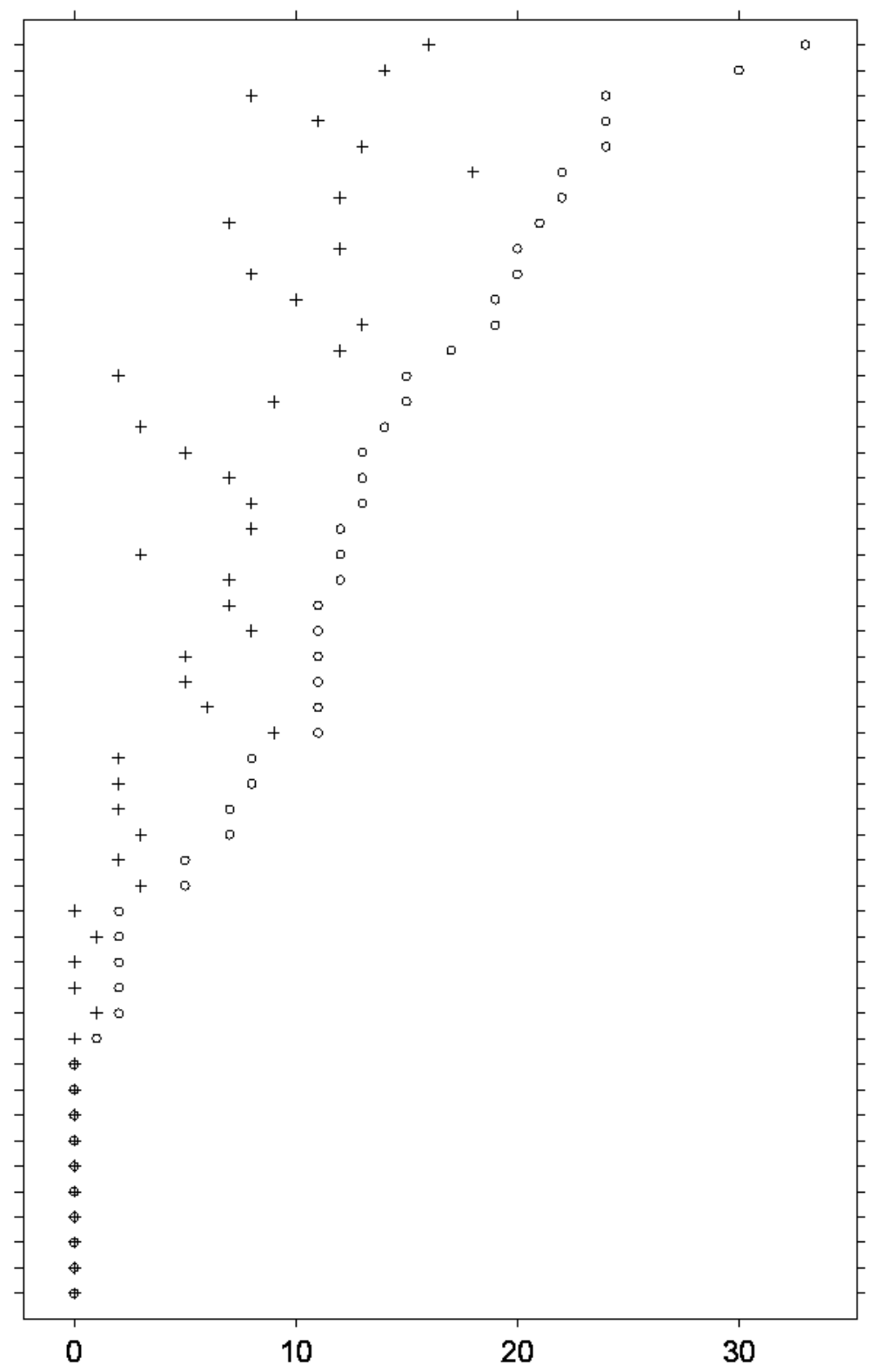

Grafik 9: Häufigkeitsverteilung der thrombembolischen Residuen 


\subsection{Methodenvergleich hinsichtlich der Diagnose CTEPH}

Im Folgenden wird ein wahrscheinlichkeitstheoretisches Modell entworfen für die Schätzung der (bedingten) Wahrscheinlichkeit, dass unter der Bedingung, in der Übersichtsangiographie (ÜA) keine thrombembolischen Residuen (tR) entdeckt zu haben, auch in der Ballon-Okklusionsangiographie (OA) keine gefunden werden (was wir damit gleichsetzen, dass tatsächlich keine vorhanden sind).

Oder: Mit welcher Wahrscheinlichkeit ist die Anzahl tatsächlich vorhandener $\mathrm{tR}$ (= in der OA entdeckbarer) gleich Null, wenn in der ÜA keine entdeckt wurden?

Herleitung des wahrscheinlichkeitstheoretischen Modells:

1. Für einen (zufällig auszuwählenden) Patienten bezeichne $N$ die zufällige Anzahl tatsächlich vorhandener tR. $N$ kann also die Werte $0,1,2, \ldots$ annehmen.

Für diesen Patienten bezeichne $X$ die zufällige Anzahl seiner in der ÜA entdeckten $\mathrm{tR}$. Ist $N=n$, so kann $\mathrm{X}$ die Werte $0,1, \ldots, n$ annehmen.

Wir postulieren, dass für jeden möglichen Wert $n$ der Zufallsvariablen $N$ jedes der tatsächlich vorhandenen $n$ tR unabhängig von den anderen $\mathrm{tR}$ mit der konstanten „Erfolgswahrscheinlichkeit“ $0<p<1$ in der ÜA gefunden wird. Dann folgt, dass die Zufallsvariable $X$ eine Binomialverteilung zur Erfolgswahrscheinlichkeit $p$ und zum Stichprobenumfang $n$ hat.

Kurz: Die bedingte Verteilung von $X$ gegeben $N=n$ ist die $\operatorname{Binomial}(n, p)$ Verteilung. Konkret bedeutet dies, dass die Wahrscheinlichkeit für $X=0$ unter der Bedingung $N=n$ gemäß

$$
P(X=0 \mid N=n)=(1-p)^{n} \quad \text { für jedes } n=0,1,2, \ldots
$$

berechnet wird. 
Wir postulieren ferner, dass die Zufallsvariable N eine Poisson-Verteilung zum Erwartungswert $\lambda$ („lambda“) besitzt. Diese Verteilungsannahme wird dadurch plausibel, dass die Anzahl aller Gefäße, in denen ein tR prinzipiell auftreten kann, sehr groß ist, aber die Wahrscheinlichkeit in jedem einzelnen Gefäß dafür sehr klein.

Konkret bedeutet dies, dass die Wahrscheinlichkeit für $\mathrm{N}=\mathrm{n}$ gemäß

$$
P(N=n)=\frac{\lambda^{n}}{n !} \cdot e^{-\lambda} \quad \text { fïr jedes } n=0,1,2, \ldots
$$

berechnet wird.

2. Gesucht ist nun in obigem Modell die bedingte Wahrscheinlichkeit $\mathrm{P}(\mathrm{N}=0 \mid \mathrm{X}=0)$.

In Worten: Die Wahrscheinlichkeit, dass die Anzahl tatsächlich vorhandener tR gleich Null ist, unter der Bedingung, dass die Anzahl der in der ÜA entdeckten tR gleich Null ist.

Unter Verwendung elementarer Regeln für das Rechnen mit bedingten Wahrscheinlichkeiten sowie der Eigenschaften der e-Funktion folgt

$$
\begin{gathered}
P(N=0 \mid X=0)=\frac{P(N=0, X=0)}{P(X=0)}=\frac{P(X=0 \mid N=0) \cdot P(N=0)}{\sum_{n=0}^{\infty} P(X=0 \mid N=n) \cdot P(N=n)} \\
=\frac{1 \cdot e^{-\lambda}}{\sum_{n=0}^{\infty}(1-p)^{n} \cdot \frac{\lambda^{n}}{n !} \cdot e^{-\lambda}}=\frac{1}{\sum_{n=0}^{\infty} \frac{((1-p) \lambda)^{n}}{n !}}=\frac{1}{e^{(1-p) \lambda}}=e^{-(1-p) \lambda}
\end{gathered}
$$

Das heißt, haben wir Schätzwerte für die (im Augenblick noch unbekannten!) Parameter $p$ und $\lambda$, so können wir die gesuchte Wahrscheinlichkeit $\mathrm{P}(\mathrm{N}=0 \mid \mathrm{X}=0)$ in diesem Modell schätzen.

3. Schätzung der Parameter $p$ und $\lambda$ : 
$\underline{\mathrm{Zu} p}$ : Dies ist die Erfolgswahrscheinlichkeit, ein tatsächlich vorhandenes tR in der ÜA zu entdecken. Es ist somit schätzbar durch den relativen Anteil der in der ÜA entdeckten tR an den in der OA entdeckten (= tatsächlich vorhandenen) tR. Als Datenbasis für diese Schätzung dient uns die in der ÜA bzw. in der OA sichtbare Anzahl an Teilungsgenerationen im linken und im rechten Unterlappen.

Für diese vier Größen nehmen wir an, dass sie Poisson-Verteilungen besitzen. Da die ÜA- und OA-Messungen an denselben Patienten vorgenommen wurden und bei einigen Patienten sogar Messwiederholungen vorliegen, handelt es sich zwangsläufig um abhängige PoissonVariablen. Diesem Sachverhalt wurde Rechnung getragen, indem zur Modellierung der zugrundeliegenden Poisson-Verteilungen ein verallgemeinertes lineares Regeressionsmodell mit „mixed effects“ (= generalized linear mixed model“ = GLMM) mit Poisson-Link verwendet wurde. Es berücksichtigte zunächst einen möglichen Effekt der Unterlappenseite (rechts oder links), der sich aber nicht als signifikant herausstellte, so dass wir die Messungen nicht nach linkem oder rechtem Unterlappen unterscheiden.

Im Rahmen dieses Modells wurde die durch die ÜA entdeckte mittlere Anzahl an tR in den Unterlappen zu 9,97 geschätzt und die durch die OA entdeckte mittlere Anzahl an tR in den Unterlappen zu 12,85. Den Quotienten aus beiden wählen wir als Schätzwert $\hat{p}$ für $p$.

$$
\hat{p}:=\frac{12.85}{9.97}=0.7760
$$

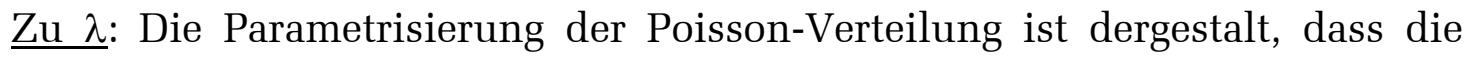
ganze Verteilung schon durch die Angabe einer einzigen der Wahrscheinlichkeiten $P(N=n)$ festgelegt ist, also insbesondere auch schon durch die Wahrscheinlichkeit $P(N=0)$. 
Dies machen wir uns zunutze, indem wir auf der Basis einer zusätzlichen, von unseren eigentlichen Daten unabhängigen (!) Stichprobe (genannt „Kontrollgruppe“) diese Wahrscheinlichkeit $P(N=0)$ schätzen und aus der Beziehung

$$
P(N=0)=e^{-\lambda} \quad \Longleftrightarrow \quad \lambda=-\ln (P(N=0))
$$

einen Schätzwert $\hat{\lambda}$ für $\lambda$ erhalten.

Als Schätzwert $\hat{P}_{0}$ für $P(N=0)$ nehmen wir den relativen Anteil derjenigen Patienten in der insgesamt 88 Individuen umfassenden Kontrollgruppe, die kein tR zeigten. Dies waren 29 Patienten, so dass sich

$$
\hat{P}_{0}=\frac{29}{88} \quad \text { und somit } \quad \hat{\lambda}:=-\ln \left(\frac{29}{88}\right)=1.11
$$

ergibt.

4. Schätzung von $P(N=0 \mid X=0)$ unter Verwendung von $\hat{p}$ und $\hat{\lambda}$ : Mit den im vorherigen Punkt ermittelten Schätzwerten erhalten wir als Schätzwert für $P(N=0 \mid X=0)$

$$
e^{-(1-\hat{p}) \hat{\lambda}}=e^{-(1-0.7760) \cdot 1.11} \approx 0.78
$$

Das heißt, in dem von uns aufgestellten Modell wird auf Basis unserer Daten die Wahrscheinlichkeit, dass ein Patient in der OA keine tR zeigt, wenn sich in der ÜA keine tR fanden, auf ca. 78\% geschätzt. Im Umkehrschluss kann man also in ca. 22\% der Fälle davon ausgehen, das die OA mindestens ein tR zu Tage fördert, das in der ÜA noch nicht gefunden wurde. 


\subsection{Komplikationen}

Im Beobachtungszeitraum der 50 in die Studie eingeschlossenen Patienten fand sich keine schwerwiegende oder gar letale Komplikation. Bei einem Patienten trat eine Herzrhythmusstörung mit synkopalem Ereignis auf, während der angiographische Katheter in der Pulmonalarterie platziert wurde. Nach Entfernung des Katheters bildete sich die Symptomatik spontan zurück. Bei einem anderen Patienten mit Niereninsuffizienz im Stadium der kompensierten Retention kam es trotz entsprechender Prophylaxe zu einem vorübergehenden Anstieg des Serumkreatinins auf bis zu 4 mg/dl im Sinne einer Kontrastmittel-induzierten Nephropathie. Hier konnte unter spezifischer Therapie ein rascher Rückgang auf das Ausgangsniveau erreicht werden. Bei einem dritten Patienten entwickelte sich im Gefolge der Venenpunktion ein schmerzhaftes Hämatom in der Leistenbeuge ohne Transfusionsbedarf. Unter lokalen Therapiemaßnahmen besserten sich die Beschwerden schnell. Bei einem weiteren Patienten kam es unmittelbar im Anschluss an die Untersuchung zu einer transitorischen ischämischen Attake (TIA). Im CT des Schädels konnten eine zerebrale Blutung und ein Hirninfarkt ausgeschlossen werden. Hier bleibt unklar, ob das Ereignis in direktem Zusammenhang mit der Pulmonalisangiographie steht, zumal echokardiographisch ein Shuntvitium als Voraussetzung für eine paradoxe Embolie ausgeschlossen werden konnte. Die geschilderten Komplikationen sind nicht speziell der Ballon-Okklusionsangiographie zuzurechnen. Sie hätten auch im Rahmen einer alleinigen, standardisierten Übersichtsangiographie eintreten können. 
Die nicht aufgelöste oder rekanalisierte Lungenembolie sowie rezidivierende Lungenembolien sind häufige Ursachen der chronischen pulmonalen Hypertonie. Die chirurgische pulmonale Thrombendarteriektomie (PTEA) ist eine effiziente Behandlungsoption, die bei ausgewählten Patienten mit Erfolg durchgeführt werden kann (Mayer et al., 1996; Moser et al., 1987; Archibald et al., 1999). Die Mortalität des Eingriffes beträgt jedoch immerhin 5-7\% (Olschewski et al., 2002; Mayer et al., 1996; Moser et al., 1987; Brett et al., 2001). Daher ist im Vorfeld der Operation eine korrekte Selektion der für den Eingriff geeigneten Patienten unabdingbar. Diejenigen mit ausschließlich peripher lokalisierten thrombembolischen Residuen müssen ebenso ausgeschlossen werden wie jene Patienten, die an einer primären pulmonalen Hypertonie leiden. Bei diesen Patienten erzielen möglicherweise Prostazykline (z.B. Epoprostenol oder Iloprost) eine Senkung des pulmonalen Gefäßwiderstandes. Allerdings kann eine kontinuierliche Prostazyklintherapie das Auftreten eines lebensbedrohlichen Lungenödemes fördern bei Patienten mit pulmonaler venookklusiver Erkrankung (PVOD) oder pulmonaler kapillärer Hämangiomatose (PCH).

Die Pulmonalisangiographie ist neben der Angio-Computertomographie eine Standardmethode, um geeignete Kandidaten für eine pulmonale Thrombendarteriektomie oder eine Prostazyklintherapie auszuwählen. Wir haben versucht, die Differentialdiagnose der genannten Erkrankungen und pathophysiologischen Bedingungen $\mathrm{zu}$ verbessern mit der selektiven, pulmonalen Ballon-Okklusionsangiographie. Im Folgenden werden zunächst die kritischen Punkte unseres Studienkollektives und der Methodenwahl diskutiert. Dann schließt sich die Wertung der erzielten Ergebnisse im Kontext anderer Forschungsdaten an mit dem Ziel, praxistaugliche Schlussfolgerungen für das diagnostische Vorgehen bei Patienten mit thrombembolischer (CTEPH) und nicht-thrombembolischer pulmonaler Hypertonie (NoCTEPH) zu formulieren. Dabei wird an Stellen, an denen es erforderlich erscheint, auf die Notwendigkeit ergänzender Studien im Sinne eines Ausblickes hingewiesen. 


\subsection{Patientenkollektiv}

In die retrospektive Studie wurden 50 Patienten mit chronischer pulmonaler Hypertonie aufgenommen, bei denen die Indikation zur Durchführung einer intraarteriellen Pulmonalisangiographie gestellt wurde. Diese Indikation ergab sich aus dem Zusammenwirken verschiedener klinischer, laborchemischer und bildgebender Befunde. Es handelt sich somit nicht um eine randomisierte Patientengruppe, was bei der Einschätzung der erzielten Ergebnisse berücksichtigt werden muss. Zu bedenken ist außerdem, dass angesichts der Vielzahl der betrachteten Parameter der Stichprobenumfang mit 50 Patienten insgesamt und insbesondere mit 37 Patienten in der CTEPH-Gruppe Anlass zu Kritik geben kann. Dass es sich trotzdem möglicherweise um ein repräsentatives Kollektiv handelt, kann aufgrund der erhobenen, beschreibenden Daten angenommen werden. So bestätigt sich in unserer Untersuchung zum Beispiel, dass Lungenembolien signifikant häufiger in der rechten Lunge gefunden werden (Wagenvoort, 1995). Pitton et al. untersuchten 2002 ein Patientenkollektiv, das im Mittel 53,8 Jahre alt war, das mittlere Alter unserer Patienten betrug 55,96 Jahre. Auch die mittleren pulmonalarteriellen Drucke lagen etwa im gleichen Niveau (45,8 bei Pitton et al versus 50,5 in unserer Studie). Trotzdem wäre es idealerweise anzustreben, für einige Parameter die Zulässigkeit der hier vorgestellten Ergebnisse durch ergänzende, prospektive und randomisierte Studien mit größerem Stichprobenumfang zu untermauern.

\subsection{Methodik}

In unserer Untersuchungsreihe wurden sowohl die Übersichtsangiographie (im Folgenden auch ÜA) als auch die Ballon-Okklusionsangiographie (im Folgenden auch OA) in digitaler Subtraktionstechnik (kurz DSA) angefertigt.

In mehreren Studien konnte für die konventionelle Übersichtsangiographie festgestellt werden, dass keine relevanten Unterschiede zwischen der digitalen und der Blattfilm-Pulmonalisangiographie hinsichtlich der Diagnosefindung einer akuten Lungenembolie bestehen (Smith et al., 2002; Johnson et al., 1998; Hagspiel et al., 1998; van Beek et al., 1996; vgl. 1.3.1). Dieser Erkenntnis stehen die praxisrelevanten Vorteile der DSA gegenüber: Die digitale Subtraktionsangiographie 
nimmt weniger Zeit in Anspruch, da vor der Bildbetrachtung die aufwändige Filmnachbearbeitung wegfällt. Die Bilder können zusätzlich zu den Einzelbildern auch als Serie oder Filmschleife begutachtet werden. Zahlreiche Nachbearbeitungswerkzeuge ermöglichen die nachträgliche Optimierung der Bildqualität einschließlich der Option, die Bilder auch im nichtsubtrahierten Originalzustand zu analysieren (Smith et al., 2002). Es bot sich an, die zu testende alternative Technik der Ballon-Okklusionsangiographie im gleichen Modus durchzuführen. So ist ausgeschlossen, dass Unterschiede in den Ergebnissen durch die Darstellungsweise hervorgerufen werden und nicht durch die Untersuchungstechnik. In einigen Arbeiten kommen andere Aufnahmetechniken für die Ballon-Okklusionsangiographie zum Einsatz. Darunter finden sich die Blattfilm-Technik (Stein et al., 1971, Wilson et al.; 1976), die es erlaubt, die Untersuchung zum Beispiel auch am Krankenbett durchzuführen, und die Cineangiographie (Ferris et al., 1984). Der Einfluss des Bilderstellungsmodus der OA ist jedoch nicht systematisch untersucht worden und somit auch in unserer Studie als unbekannt einzustufen.

Im Beobachtungszeitraum der 50 Studienpatienten traten vier Minor-Komplikationen auf, die nicht unbedingt durch die zusätzliche Ballon-Okklusionsangiographie hervorgerufen wurden (Hämatom, Niereninsuffizienz, Arrhythmie und TIA, vgl 3.7). Vielmehr handelte es sich dabei um allgemeine Komplikationen der konventionelle Pulmonalisangiographie (Nilsson et al., 1998). Jacobson berichtet 1963 über die Gefahr einer lokalisierten pulmonalen Nekrose aufgrund einer zu hohen Kontrastmittelkonzentration bei der Ballon-Okklusionsangiographie. Stein empfiehlt diesbezüglich 1971, das injizierte Kontrastmittel unverzüglich durch eine nachfolgende Kochsalzinjektion auszuschwemmen. Unter Beachtung dieser Vorsichtsmaßnahme trat eine solche Komplikation in unserem Studienkollektiv nicht auf. Sumita (1995) berichtet über die Ruptur einer Pulmonalarterie mit Ausbildung von Hämoptysen im Rahmen einer Ballon-Okklusionsangiographie. In der Angiographie war ein Kontrastmittelextravasat erkennbar. In jüngerer Vergangenheit ereignete sich eine ähnliche Major-Komplikation außerhalb der vorgestellten Studie in unserem Institut. Möglicherweise handelte es sich um eine Verletzung des Lungenparenchymes durch vorausgegangene Drahtmanipulationen. Es ist somit immer zu beachten, dass die Ballon-Okklusionstechnik das Risiko der Pulmonalisangiographie zwar nur geringfügig, aber doch erkennbar erhöht. 


\subsection{Chronische thrombembolische pulmonale Hypertonie}

\subsubsection{Thrombembolische Residuen}

\section{$\underline{\text { Pulmonalishaupstamm und Lappenarterien }}$}

Die chirurgische pulmonale Thrombendarteriektomie (PTEA) ist bei der CTEPH überwiegend dann möglich, wenn die Pulmonalisangiographie thrombembolische Residuen in den Pulmonalarterienhauptstämmen, den Lappenarterien oder wenigstens in den proximalen Segmentarterien zeigt (Fedullo et al., 2001).
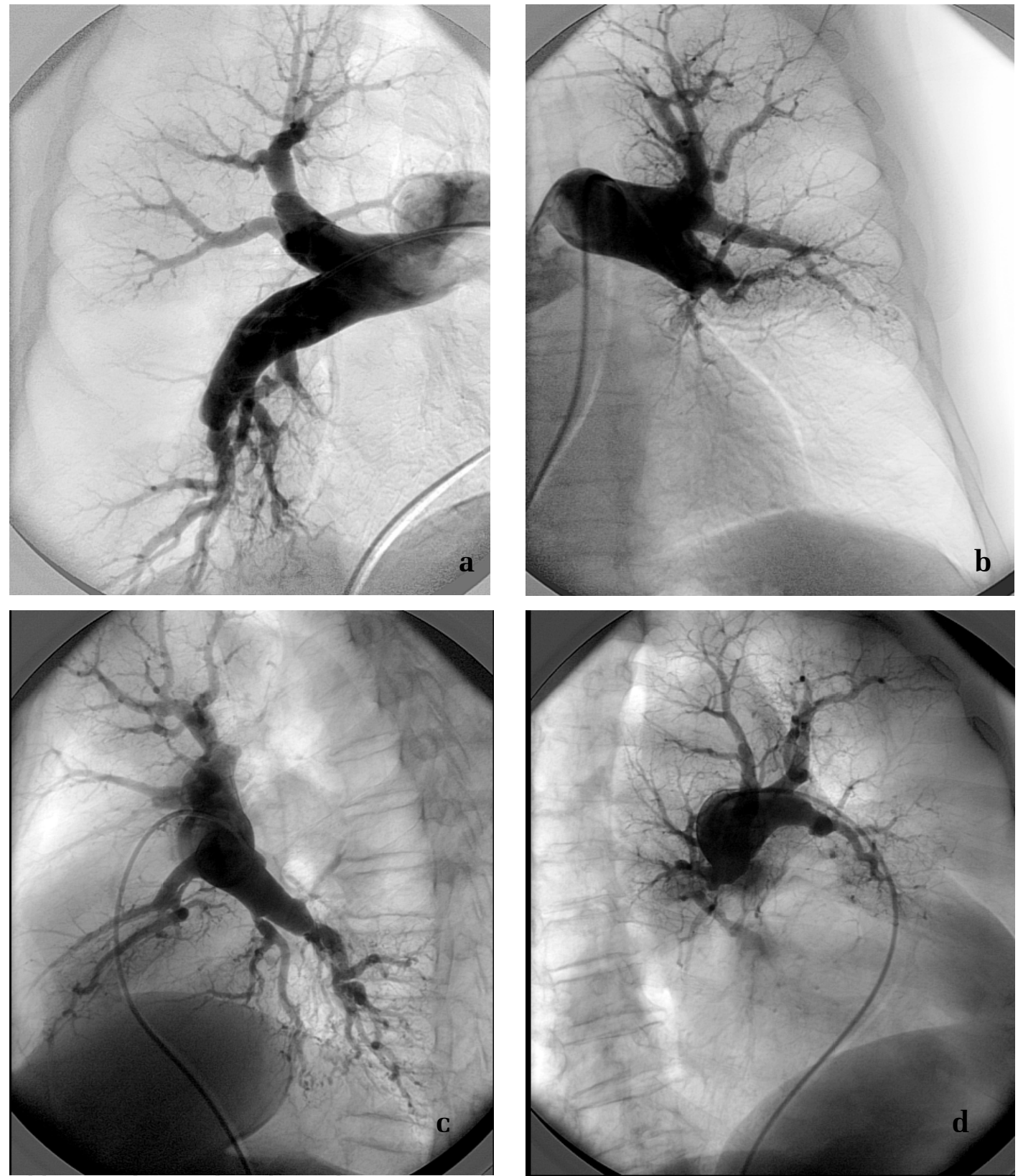

Abb. 16 a-d zeigt die Ubersichtsangiographie in a.-p. und seitlicher Projektion bei einem Patienten mit proximalem Befallsmuster bei CTEPH. Multiple Verschlüsse und Stenosen von Lobar- und Segmentarterien sind erkennbar. Die linke Unterlappenarterie fehlt komplett, auf der rechten Seite sind einige Segmentarterien nicht durchblutet. 
Dabei finden sich Stenosen, Verschlüsse, Strickleitersysteme (Webs und Bands) sowie Wandauflagerungen in den proximalen Pulmonalarterien. In unserer Untersuchung waren die Pulmonalarterienhauptstämme und die Lappenarterien immer bereits in der Übersichtsangiographie gut beurteilbar. Bei 21 Patienten fanden sich thrombembolische Residuen in diesen Gefäßen. 16 mal wurde die Indikation zur PTEA gestellt (76\%), in 12 Fällen wurde sie erfolgreich durchgeführt (57\%). Die Zahlen zeigen, dass einerseits das proximale Befallsmuster (Abb. 16) in der Pulmonalisangiographie zwar eine erforderliche Bedingung in der Auswahl von Kandidaten für eine PTEA ist, dass andererseits aber weitere Faktoren wie allgemeine Operabilität und pulmonalarterielle Druckverhältnisse einschließlich ihrer Beeinflussbarkeit durch medikamentöse Therapie (Zonzin et al., 2003) die Indikationsstellung entscheidend beeinflussen.

\section{$\underline{\text { Segmentarterien }}$}

Der exakten Darstellung der Segmentarterien kommt bei der Entscheidung, ob eine Thrombendarteriektomie möglich ist oder nicht, eine große Bedeutung zu. Bei 32 der 37 Patienten $(86,5 \%)$ mit CTEPH in unserer Untersuchung fanden sich thrombembolische Residuen in den Segmentarterien der rechten Lunge. Bei 26 (70\%) Patienten fanden sich thrombembolische Veränderungen der linken Lunge. Es ergibt sich eine signifikante Bevorzugung der rechten Seite. Dies ist vereinbar mit Angaben in der Literatur. Wagenvoort (1995) zum Beispiel beschreibt bei CTEPH das zahlreichere Vorkommen thrombembolischer Residuen in der rechten Lunge. Die Ballon-Okklusionsangiographie zeigt auf dem Niveau der Segmentarterien keine statistisch signifikante Überlegenheit im Nachweis thrombembolischer Residuen insgesamt. Aber immerhin macht sie in 10,8\% aller Fälle unabhängig von der Genese der pulmonalen Hypertonie Webs und Bands sichtbar, die in der Übersichtsangiographie auch retrospektiv nicht nachweisbar sind. Bezogen auf die Patienten mit CTEPH alleine ist dieser Unterschied deutlicher $(15,4 \%)$. Dies liegt nicht nur daran, dass die Ballon-Okklusionsangiographie Gefäßterritorien überlagerungsfrei abbildet (Abb. 17, S. 63). 


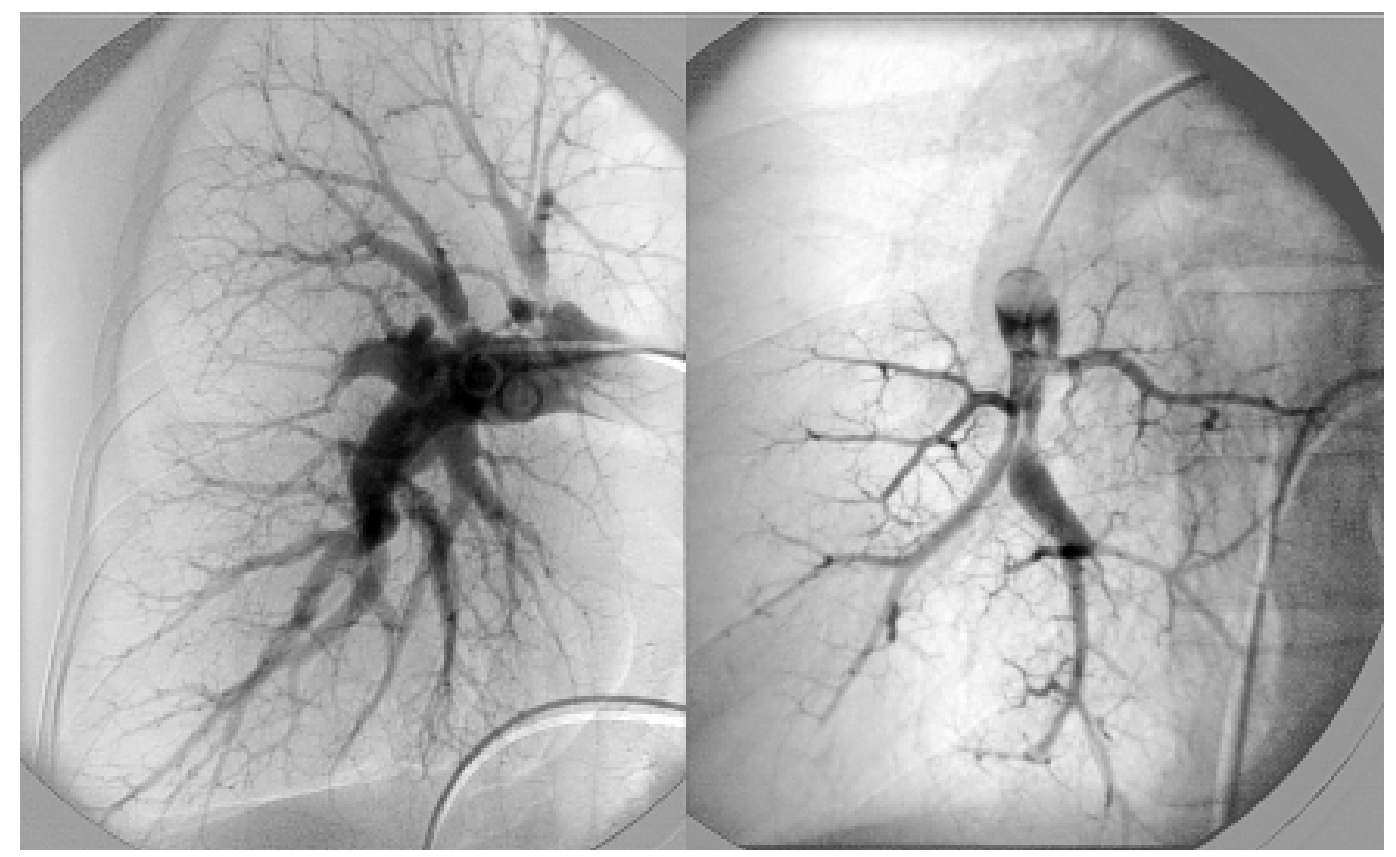

Abb. 17: Gegenüberstellung einer Übersichtsangiographie der rechten Lunge in RAO-Projektion (rechts) und der dazugehörigen Ballon-Okklusionsangiographie eines Unterlappensegmentes der rechten Lunge (links). Nur in der Ballon-Okklusionsangiographie ist erkennbar, dass Webs und Bands bereits in der Segmentarterie beginnen.

Sie verbessert die Bildgebung der Pulmonalarterien zusätzlich aufgrund der starken Vergrößerung und insbesondere durch den höheren intraluminalen Kontrast ohne Verdünnungseffekt als Folge von nachströmendem, nichtkontrastiertem Blut.

Auch Bynum et al. sehen 1979 die entscheidenden Vorteile der Okklusionsangiographie insbesondere darin, dass der Verschluss einer proximalen Arterie durch einen Ballon eine hohe Konzentration an Kontrastmittel in den peripheren Verästelungen ermöglicht, während Verdünnung und Auswaschen des Kontrastes solange vermeidbar sind, bis der Ballon deflatiert ist. Außerdem betonen die Autoren auch die minimierte Überlappung von benachbarten Gefäßen vor allem in den basalen Lungenabschnitten als einen wichtigen Vorteil der BallonOkklusionsangiographie. Die geschilderten Effekte des Verfahrens offenbaren sich in unserer Studie auch in der guten Detailerkennbarkeit der Pulmonalarterien. In allen Lungenabschnitten können auf den Bildern der Ballon-Okklusionsangiographie signifikant mehr Aufzweigungsgenerationen gezählt und somit auch beurteilt werden als in der herkömmlichen Übersichtsangiographie.

Unsere Studie ist in zweifacher Hinsicht retrospektiv angelegt: Einerseits handelt es sich um ein vorselektiertes (also nicht randomisiertes) Patientenkollektiv, in dem die möglicherweise operable CTEPH überrepräsentiert ist. Des Weiteren wurde der 
Vergleich der beiden Untersuchungsverfahren nicht als Doppelblindstudie durchgeführt. Vielmehr wurde ausgehend von den Befunden der Ballon-Okklusionsangiographie als vermeintlich exakterem Verfahren die Übersichtsangiographie retrospektiv ausgewertet. In Kenntnis der Befunde aus der Ballon-Okklusionsangiographie konnten Auffälligkeiten in der Übersichtsangiographie leichter als pathologisch eingestuft werden. Die brillantere Abbildungsqualität der BallonOkklusionsangiographie steigert dadurch die Befundungssicherheit, was sich in unserer Versuchsanordnung nicht in Zahlen ausdrücken lässt. Es ist jedoch bekannt, dass die konventionelle Pulmonalisangiographie eine begrenzte Untersucher-Übereinstimmung sowohl intra- als auch interindividuell aufweist. Stein et al. beschreiben 1999 bei der Befundung von Lungenembolien in der konventionellen Pulmonalisangiographie durch mindestens zwei unabhängige Untersucher im Segmentarterienniveau eine Co-Positivität von 90\%. Die Übereinstimmung nimmt auf Höhe der Subsegmentarterien auf $66 \%$ ab. Die intraindividuelle Übereinstimmung bei mehrfacher Befundung der gleichen Pulmonalisangiographie in definierten zeitlichen Abständen durch einen Untersucher beträgt nur 89\% hinsichtlich des Nachweises oder Ausschlusses einer akuten Lungenembolie (Stein, 1996). Durch die eindeutigere Abbildung thrombembolischer Residuen in der Ballon-Okklusionsangiographie können diese Werte verbessert und somit eine größere Befundungssicherheit erzielt werden. Wilson und Bynum beschreiben diesen Vorteil der Okklusionsangiographie 1976 für den Nachweis akuter Lungenembolien: Auch sie beobachteten, dass Füllungsdefekte und Gefäßabbrüche in den größeren Pulmonalgefäßen, die bereits auf der Übersichtsangiographie erkennbar sind, mit größerer Klarheit dargestellt und mit höherem Maß an Sicherheit identifiziert werden. Die Ballon-Okklusionsangiographie kann also auch bei der Frage nach der Operabilität einer thrombembolisch bedingten pulmonalen Hypertonie wertvolle Befunde erbringen, wenn es darum geht, den proximalen Beginn der Emboliefolgen in den Segmentarterien mit größtmöglicher Sicherheit zu identifizieren. Dadurch kann man möglicherweise in manchen Fällen, in denen die Übersichtsangiographie die Ausdehnung der Veränderungen in den Segmentarterien nicht sicher zeigt, auf die präoperative Angioskopie verzichten (vgl. 1.3.6). Für das praktische Vorgehen kann man aus unseren Zahlen und Beobachtungen schlussfolgern, dass bei solchen Patienten, bei denen die thrombembolische Genese der pulmonalen Hypertonie bereits gesichert ist, die Frage nach Operabilität der 
Emboliefolgen meistens durch die konventionelle Übersichtsangiographie alleine beantwortet werden kann. Dem Untersucher ist jedoch mit der Ballon-Okklusionsangiographie ein Instrument an die Hand gegeben, um fragliche Befunde insbesondere auf Höhe der Segmentarterien, innerhalb derer die Grenze zwischen Operabilität und Inoperabilität gezogen wird, eindeutiger herauszuarbeiten.

Der folgende Fall demonstriert dies: Abbildung 18 zeigt in der Übersichtsangiographie einen Verschluss der posterobasalen Segmentarterie links mit fehlender Kontrastierung der peripheren Gefäßaufzweigungen in diesem Segment.
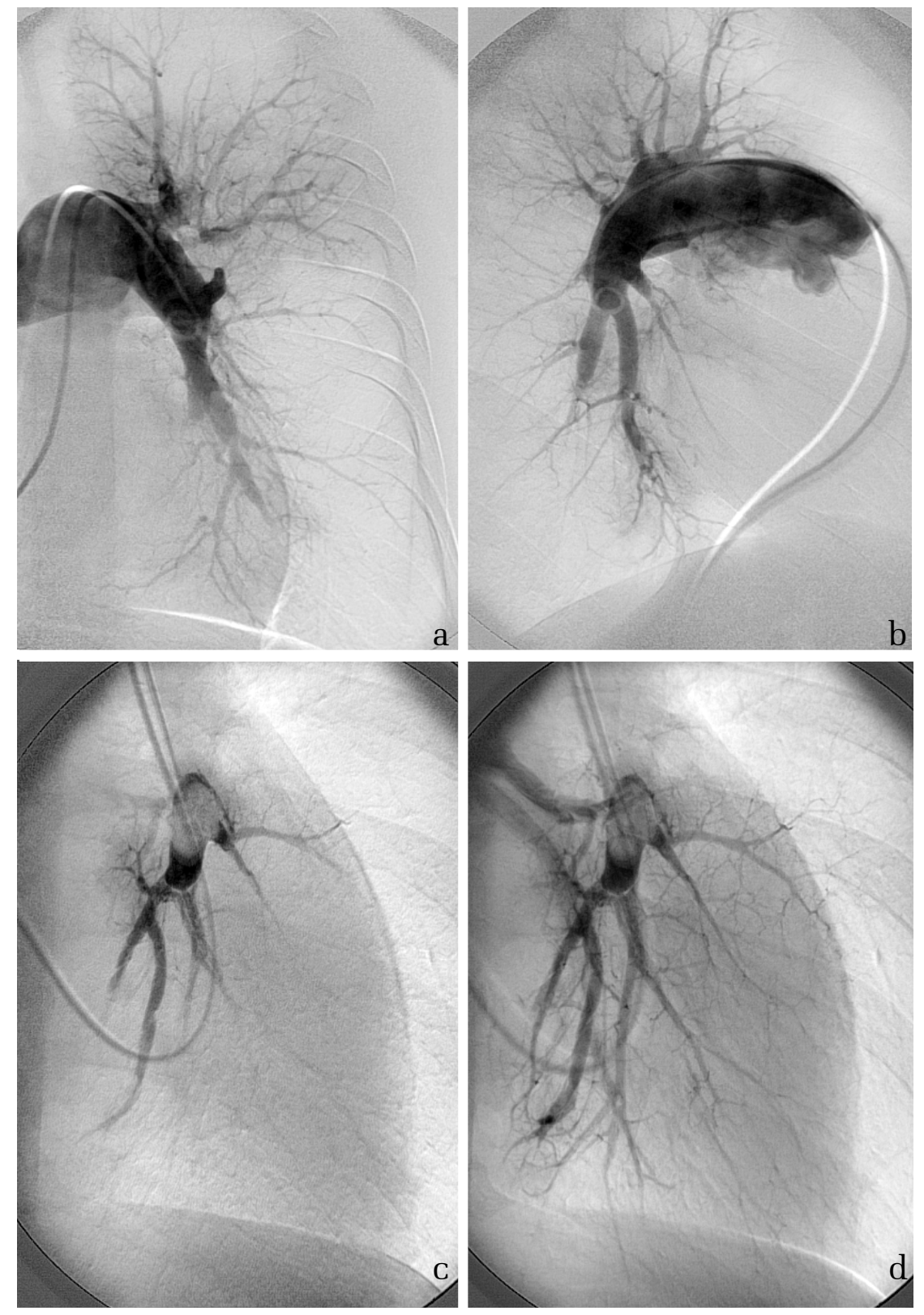

Abb. 18 a-d verdeutlicht im Falle eines Unterlappensegmentes links, dass die Ballon-Okklusionsangiographie (c-d) Gefäße darstellen kann, die in der Übersichtangiographie (a-b) verschlossen erscheinen. 
Nach Einführen des Okklusionsballons in den Stumpf der verschlossenen Segmentarterie zeigt die Okklusionsangiographie relativ kaliberstarke periphere Aufzweigungen, die auf der Übersichtsangiographie nicht erkennbar waren. Diese Gefäße speisen aber kein regelrechtes Lungenparenchym, sondern gewinnen relativ schnell Anschluss an das Venensystem und führen zu einer frühzeitigen venösen Füllung. Die ersten Aufnahmen dieser Serie (c) zeigen unmittelbar distal des Ballons über eine Strecke von 1-2 cm strähnige, rekanalisierte, sehr dünne Spalträume in dem weitgehend verschlossenen Gefäßsegment. In diesem Fall wird besonders deutlich, dass mit Hilfe der Ballon-Okklusionsangiographie Gefäßsegmente dargestellt werden können, die in der Übersichtsangiographie verschlossen erscheinen.

\section{Subsegmentarterien, Gefäße 6. Ordnung und Peripherie}

Klepetko et al. weisen in einer Publikation aus dem Jahre 2004 darauf hin, dass mit zunehmender Erfahrung in der Operationstechnik die Endarteriektomie auch in den subsegmentalen Pulmonalarterien möglich ist. Das bedeutet, dass die Diagnostik in diesem Bereich verfeinert werden muss, um dem Chirurgen ein möglichst genaues Abbild über das Ausmaß und die Lokalisation der thrombembolischen Veränderungen in diesen Gefäßen zu präsentieren. Mit Hilfe der Ballon-Okklusionstechnik kann die diagnostische Aussagekraft der Pulmonalisangiographie auf Höhe der Subsegmentarterien verbessert werden. Die für die CTEPH spezifischen Merkmale Stenosen, Webs und Bands sowie Verschlüsse sind signifikant häufiger nachweisbar. Die relativen Häufigkeiten, mit denen sie mehr thrombembolische Residuen erkennt als die Übersichtsangiographie, betragen 22\% (Stenosen), 46\% (Webs und Bands) bzw. 10\% (Verschlüsse) . In den Gefäßen sechster Ordnung nehmen diese Wahrscheinlichkeiten, dass die Ballon-Okklusionsangiographie mehr zeigt als die Übersichtsangiographie, auf 38\%, 62\% bzw. $18 \%$ und in der Peripherie auf 56\%, 58\% bzw. 40\% zu. Betrachtet man die Patienten mit einer CTEPH isoliert, so ist die Ballon-Okklusionsangiographie in den Subsegmentarterien, den Gefäßen sechster Ordnung und in der Peripherie signifikant überlegen hinsichtlich des Nachweises thrombembolischer Residuen.

In vorangegangenen Studien wurde dies bereits für den Nachweis akuter peripherer Lungenembolien festgestellt. Stein et al. konnten bereits 1971 kleinste periphere Embolien in Gefäßen mit einem Durchmesser von ca. 1,5 mm nachweisen. Wilson 
und Bynum stellten 1976 unter anderem fest, dass die Ballon-Okklusionsangiographie kleine subsegmentale Äste mehrere Generationen distal der Segmentarterien besser und eindeutiger darstellt als die konventionelle Übersichtsangiographie. Dies führe dazu, dass kleine periphere Embolien zuverlässiger nachgewiesen, aber auch ausgeschlossen werden können. Ferris et al. weisen 1984 darauf hin, dass bei der Suche nach frischen Embolien die Ballon-Okklusionsangiographie eine wertvolle Erweiterung der konventionellen Angiographie ist. Insbesondere bei positiver Szintigraphie mit kleinen subsegmentalen Perfusionsausfällen sei sie in der Lage, kleine periphere Embolien gezielt nachzuweisen, wenn sie in der Standarduntersuchung verborgen blieben. Greene et al. fanden 1983 bei Patienten mit akutem respiratorischem Versagen (ARDS) in der BallonOkklusionsangiographie Gefäßveränderungen wie thrombotische Füllungsdefekte oder Verschlüsse in peripheren Verästelungen bis zu einem minimalen Durchmesser von $5 \mathrm{~mm}$. In unserer Studie konnte nachgewiesen werden, dass die Erkenntnisse aus diesen vorangegangenen Untersuchungen auch für die häufig subtileren Gefäßveränderungen der chronischen thrombembolischen pulmonalen Hypertonie zutreffen. Die Ballon-Okklusionsangiographie wird jedoch die konventionelle Übersichtsangiographie nicht ersetzen können, da sie nie den gesamten pulmonalen Gefäßbaum abbildet. Vielmehr ermöglicht sie in mehr oder weniger zufällig ausgewählten Lungenarealen oder gezielt dort, wo die Szintigraphie Auffälligkeiten zeigte, eine Verfeinerung der Diagnostik.

\section{$\underline{\text { Sensitivität hinsichtlich der Diagnose CTEPH }}$}

Ferris et al. beschreiben 1984, dass die Ballon-Okklusionsangiographie bei vier Patienten aus 38 positiven Untersuchungen (10,5\%) die akute Lungenembolie nachweisen konnte, die in der Standardtechnik nicht sichtbar war. In unserem Studienkollektiv fand sich kein Patient, bei dem die Ballon-Okklusionsangiographie wenigstens eines und die Übersichtsangiographie kein thrombembolisches Residuum zeigte. Dies bedeutet jedoch nicht, dass die Ballon-Okklusionsangiographie die Sensitivität der Pulmonalisangiographie bezüglich der Diagnose CTEPH nicht verbessert. Um trotzdem eine Aussage über die Sensitivität der Okklusionstechnik im Vergleich mit der Übersichtsangiographie machen zu können, wurde ein wahrscheinlichkeitstheoretisches Modell auf der Basis der besseren Detailerkennbarkeit in der Ballon-Okklusionsangiographie hergeleitet (vgl. 
3.6). Die Berechnung erbrachte als Schätzwert, dass bei ca. jedem fünften Patienten eines im Diagnosenspektrum vergleichbaren Kollektives unabhängig von der Ursache der pulmonalen Hypertonie die Ballon-Okklusionsangiographie thrombembolische Residuen nachweist, wenn die Übersichtsangiographie negativ ist. Diese Hypothese könnte durch eine weiterführende, randomisierte Doppelblindstudie überprüft werden. Für die Praxis kann man jedoch festhalten, dass die ergänzende Ballon-Okklusionsangiographie dann durchgeführt werden sollte, wenn die nichtinvasive Diagnostik eine CTEPH wahrscheinlich macht, die konventionelle Übersichtsangiographie jedoch keine Emboliefolgen erkennen lässt. Tatsächlich fand sich bei der Durchsicht des Kontrollkollektives (also bei 88 Patienten zusätzlich zum Studienkollektiv von 50 Patienten, vgl. 2.1) ein Patient mit der Diagnose CTEPH, bei dem die Übersichtangiographie auch in Kenntnis der Lokalisation der thrombembolischen Residuen aus der Ballon-Okklusionsangiographie negativ ist. Ferris (1984) beschreibt, dass bei vier Patienten von insgesamt 38 positiv untersuchten Patienten die Lungenembolie nicht in der Übersichtsangiographie sichtbar war, wohl aber in der Ballon-Okklusions-Cineangiographie.

\subsubsection{Parenchymminderperfusion}

Stenosen und Verschlüsse durch Thrombembolien rufen eine Minderperfusion des nachgeschalteten Lungenparenchymes hervor. Dies ist auch als angiographisches Symptom in der Pulmonalisangiographie erkennbar (Ludwig, 1983). Es zeigt sich dann in der parenchymalen Phase eine reduzierte oder fehlende Kontrastanfärbung des Lungengewebes entweder generalisiert oder regional. Ryan et. al. beschreiben 1988, dass die Szintigraphie regelmäßig dazu verleitet, das Ausmaß der Parenchymminderperfusion bei bekannter Lungenembolie im Vergleich zu Angiographie und hämodynamischen Parametern zu unterschätzen (Ryan, 1988; Moser, 1992). Außerdem konnte bisher keine strikte Korrelation zwischen der angiographisch oder szintigraphisch quantifizierten Minderperfusion und dem Schweregrad der hämodynamischen Beeinträchtigung gefunden werden (Ryan, 1988). Unsere Daten lassen ebenfalls keine sichere Beziehung zwischen Parenchymminderperfusion und gemessenen pulmonalarteriellen Drucken erkennen. Es fand sich jedoch, dass die angiographische Minderkontrastierung des Lungen- 
parenchymes bei den CTEPH-Patienten signifikant ausgeprägter war als bei anderen Patienten mit pulmonaler Hypertonie. Allerdings hatten die CTEPHPatienten, bei denen die Indikation zu einer pulmonalen Thrombendarteriektomie gestellt wurde, keine signifikant deutlichere Minderkontrastierung als solche, die nicht operiert werden konnten.

\subsubsection{Anastomosen und Kollateralen}

Die Darstellung einer Kollateralisation von thrombembolisch verschlossenen Pulmonalarterien mit peripherer Wiederauffüllung des Originalgefäßes ist bisher nur mit dem aufwändigen Verfahren der intraarteriellen Angiographie der Bronchialarterien gelungen. Nach selektiver Kontrastmittelinjektion in die Bronchialarterien von Patienten mit CTEPH konnten Endrys et al. 1997 die bemerkenswerte bronchialarterielle Dilatation und die bronchopulmonale Kollateralzirkulation zeigen. Der bronchialarterielle Fluss erreichte bis zu 30\% des systemischen Blutflusses, um Pulmonalgefäße distal thrombembolischer Verschlüsse wieder aufzufüllen. Die Autoren schlussfolgern aus ihren Beobachtungen, dass die thorakale Aortographie oder die selektive Bronchialarteriographie hilft, Patienten mit CTEPH von denjenigen mit anderen Ursachen der chronischen pulmonalen Hypertonie zu unterscheiden. Diese Techniken ermöglichen jedoch nicht die gleichzeitige Abbildung des gesamten pulmonalarteriellen Gefäßbaumes.

Bookstein beobachtete 1969 in der pulmonalen Ballon-Okklusionsangiographie Cluster aus geschlängelten 0,5 bis $1 \mathrm{~mm}$ großen Gefäßen in Gebieten distal von akuten Lungenembolien. Eine Verbindung zu benachbarten Pulmonalarterien ging daraus nicht hervor. Er deutete diese Gefäße als dünnwandige Kanäle, die hypertrophierten Kapillaren ähnelten.

Die Ballon-Okklusionsangiographie zeigte in unserer Studie bei Patienten mit CTEPH häufig eine Kollateralisation verschlossener Pulmonalarterien aus benachbarten, noch durchbluteten Arterien (vgl. 3.5, Abb. 14-15). Dies ist das erste Mal, dass Anastomosen zwischen Pulmonalarterien radiologisch nachgewiesen wurden. Gleich einer anatomischen Doktrin wurden Pulmonalarterien bisher als „funktionelle Endarterien“ beschrieben, die keine Anastomosen untereinander formen (Duncker, 1985). Nur die Verbindungen zu den Bronchialarterien sind be- 
kannt (vgl. 1.1). Möglicherweise dienen hypertrophierte, zum Teil retrograd durchflossene Bronchialarterien als verbindender Schenkel zwischen durchbluteten und verschlossenen Pulmonalarterien. Diejenigen Anastomosen, die zu einer Wiederauffüllung von zentral verschlossenen Pulmonalarterien führen (also echte Kollateralen), erweisen sich in unserer Studie als spezifisch für die CTEPH. Dies untermauert die eingangs zitierte Feststellung (Endrys et al., 1997), dass der Nachweis von Kollateralen diagnostische Aussagekraft besitzt. Im Unterschied zur genannten Arbeit ist es jetzt darüber hinaus gelungen, diese Kollatateralen im Rahmen einer Erweiterung der Pulmonalisangiographie darzustellen.

In einer Arbeit von Kauczor et al. aus dem Jahr 1994 wurde beschrieben, dass die Hypertrophie und Schlängelung von Bronchialarterien in der Computertomographie alleine statistisch signifikante Prädiktoren für das Überleben der Patienten nach PTEA sind. Wir stellten einen Zusammenhang zwischen dem angiographischen Nachweis von kollateralisierenden Anastomosen und der Höhe des pulmonalarteriellen Mitteldruckes fest. Dieser war bei Patienten mit solchen Anastomosen im Mittel ca. 7,3 mmHg höher als bei den CTEPH-Patienten ohne dieses Merkmal. Es besteht also ein Zusammenhang zwischen dem Auftreten kollateralisierter Verschlüsse und hämodynamischen Gegebenheiten. Ob sich hieraus auch prognostische Aussagen ableiten lassen, müssen ergänzende prospektive Studien untersuchen.

Anastomosen zu subpleuralen Bronchialarterien ohne Verbindung in benachbarte Pulmonalarterien (vgl. 2.5.2, Abb. 9a) sind wahrscheinlich nicht spezifisch für eine bestimmte Grunderkrankung der pulmonalen Hypertonie. Sie treten am häufigsten bei der CTEPH, sporadisch jedoch auch bei anderen Formen auf. Darüber hinaus sind diese Anastomosen gelegentlich auch in der Übersichtsangiographie erkennbar.

\subsection{Nicht-thrombembolische chronische pulmonale Hypertonie}

Die Behandlung mit Prostazyklinen (z.B. Epoprostenol oder Iloprost) kann den pulmonalen Gefäßwiderstand reduzieren bei Patienten mit chronischer pulmonaler Hypertonie, wenn die chirurgische Thrombendarteriektomie nicht durchführbar ist oder wenn eine thrombembolische Ursache der pulmonalen Hypertonie ausge- 
schlossen werden konnte (Olschewski, 2002). Wir haben versucht, morphologische Unterschiede der Gefäßsysteme dieser Patienten zu entdecken. Während die zentralen Pulmonalgefäße bei allen Patienten erweitert sind, unterscheiden sich die Muster der peripheren Verzweigungen zu Teil erheblich.

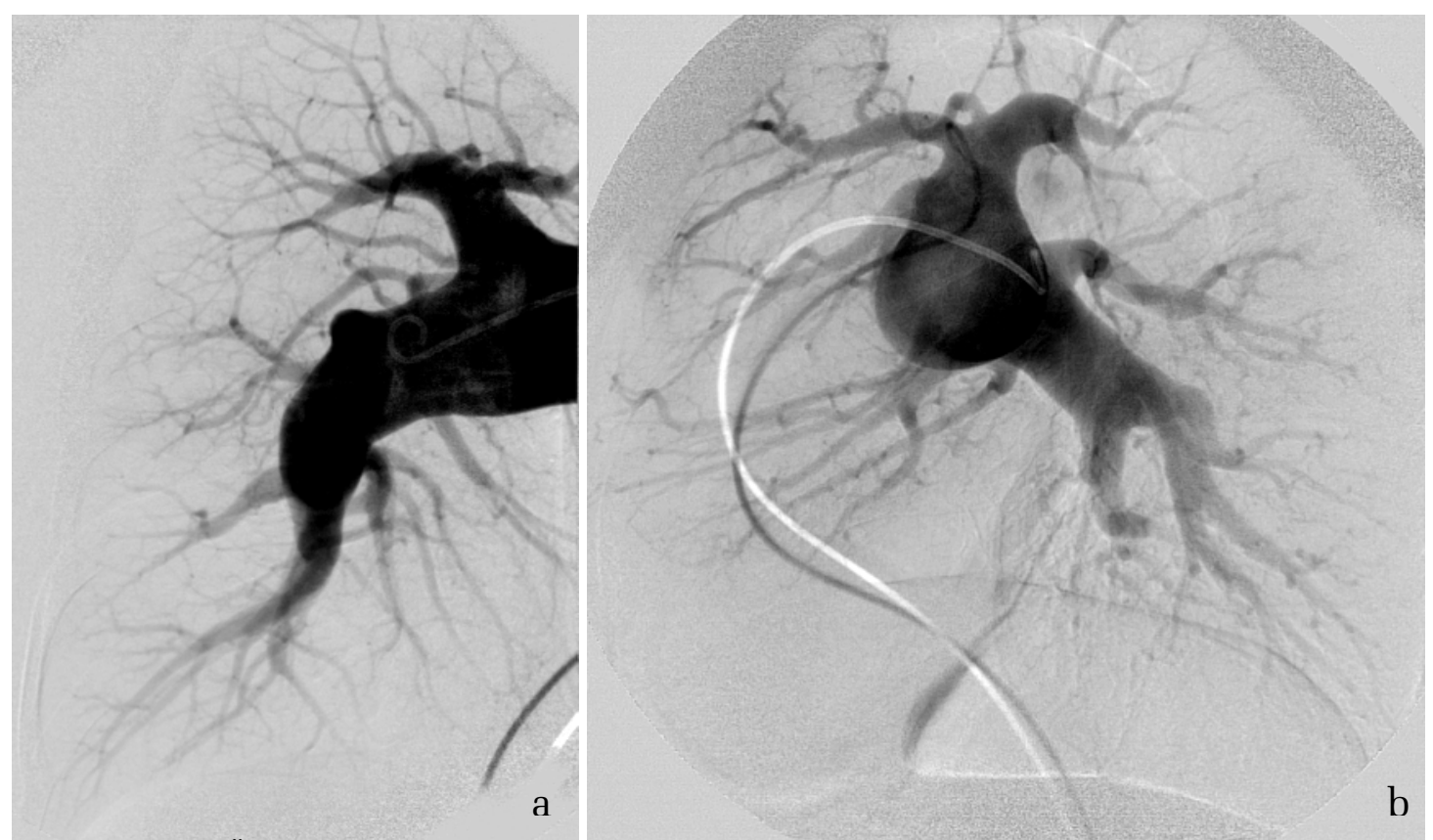

Abb. 19 a-b: Übersichtsangiographie in a.p. (a) und seitlicher Projektion (b) bei primärer pulmonaler Hypertonie: Typische Dilatation der zentralen Pulmonalarterien

Bei der primären pulmonalen Hypertonie sind als angiographische Zeichen in der Peripherie Kalibersprünge, subsegmentale Gefäßabbrüche, fadenförmige und korkenzieherartig gewundene Gefäße sowie gelegentlich subpleurale Kollateralgefäße bekannt (Frazier, 2000). Nach unserer Beobachtung erlaubt die Ballon-Okklusionsangiographie die Unterscheidung von zwei peripheren Verzweigungstypen: Einerseits die Verplumpung und korkenzieherartige Schlängelung der subsegmentalen und peripheren Gefäße („plumpe Wurzel“; vgl. 2.5.2, Abb. 8a), andererseits die Kaliberabnahme und Rarefikation der Seitäste („dürre Birke“, vgl. 2.5.2; Abb. 8b). Bislang können nur Vermutungen angestellt werden über die Ätiologie und die pathophysiologische Bedeutung dieser Beobachtungen. Möglicherweise sind die verschiedenen Ausprägungen verbunden mit der Zeitdauer seit Erkrankungsbeginn oder mit bestimmten hämodynamischen Konstellationen. In unserem Studienkollektiv ist die Zahl der Patienten zu gering für eine exakte statistische Analyse. In folgenden, prospektiven Studien soll geklärt werden, ob sich aus dem Nachweis der verschiedenen Verzweigungstypen ätiologische, therapeutische (z.B. 
Ansprache auf bestimmte Medikamente) oder prognostische Schlüsse ziehen lassen.

Die abrupte Verjüngung peripherer Gefäße (Kalibersprünge) sowie peripher gelegene Gefäßabbrüche als unspezifische Zeichen der pulmonalen Hypertonie waren auch in unserer Studie in der Ballon-Okklusionsangiographie häufiger und eindeutiger erkennbar als in der Übersichtsangiographie.

Prostazykline verbessern die pulmonale Hypertonie in den meisten Fällen, es gibt jedoch auch Ausnahmen. In der hochaufgelösten Computertomographie kennt man pathologische Zeichen, die streng mit dem Auftreten eines lebensbedrohlichen Lungenödemes unter Prostazyklintherapie korrelieren (Resten, 2002). Unscharf begrenzte noduläre Verdichtungen, Verdickung der Interlobularsepten, Pleuraergüsse und vergrößerte Lymphknoten lenken dabei den Verdacht auf das Vorliegen einer pulmonalen venookklusiven Erkrankung (PVOD) oder einer primären kapillären Hämangiomatose $(\mathrm{PCH})$. In solchen Fällen wird die histologische Diagnosesicherung nach offener Lungenbiopsie empfohlen, bevor die Prostazyklintherapie begonnen wird. In Einzelfällen sind auch angiographische Zeichen der PVOD beschrieben worden. Shackelford (1977) und Matsuomo (1993) fanden eine Erweiterung der großen zentralen Pulmonalarterien und des rechten Ventrikels, eine verlängerte Parenchymkontrastierung und eine verzögerte Füllung der Lungenvenen. Die wenigen Patienten mit PVOD oder PCH als Formen der postkapillären pulmonalen Hypertonie in unserer Studie fielen durch eine eigenartige Kontrastierungsdynamik in der Ballon-Okklusionsangiographie auf: Es füllen sich die Pulmonalarterien bis in die kleinsten Verästelungen hinein regelrecht mit Kontrastmittel, und im Anschluss an diese arterielle Kontrastierungsphase kommt es $\mathrm{zu}$ einem normalen Kontrastmittelabstrom über völlig normale Lungenvenen. Eine dazwischengeschaltete Parenchymanfärbung konnte nicht beobachtet werden (vgl. 3.3 Abb. 11b). Möglicherweise handelt es sich hierbei um ein spezifisches Zeichen der parenchymatösen Erkrankungen und insbesondere der postkapillären Vaskulopathie. In folgenden Untersuchungen, die sich über einen größeren Beobachtungszeitraum erstrecken, kann diese Hypothese möglicherweise bestätigt werden. Dies wäre wünschenswert, da man dann möglicherweise auf die invasivere offene Lungenbiopsie verzichten könnte. 
Die chronische pulmonale Hypertonie ist eine seltene Erkrankung, die heute noch als unheilbar gilt. Die therapeutischen Möglichkeiten haben sich in den letzten Jahren rasant weiterentwickelt und orientieren sich an der Pathogenese und an den Befunden aus der bildgebenden Diagnostik. Diese muss in ihren Möglichkeiten der differentialdiagnostischen Eingrenzung zugrundeliegender Ursachen der pulmonalen Hypertonie Schritt halten. Insbesondere die Ergebnisse der intraarteriellen Pulmonalisangiographie als Referenzmethode in der Bildgebung der Lungenstrombahn haben meist unmittelbare therapeutische Konsequenz, z.B. ob ein Patient für eine pulmonale Thrombendarteriektomie gegeignet ist oder nicht. Wir haben überprüft, ob die selektive pulmonale Ballon-Okklusionsangiographie als Erweiterung der standardisierten Übersichtsangiographie die Differentialdiagnose z.B. der embolischen (CTEPH) gegenüber der nichtembolischen (NoCTEPH) Erkrankung verbessert.

$\mathrm{Zu}$ diesem Zweck wurden Untersuchungen von 50 Patienten bestehend aus jeweils einer konventionellen Übersichtsangiographie und der ergänzenden Ballon-Okklusionsangiographie retrospektiv nach einem standardisierten Studienprotokoll ausgewertet. Zunächst wurde eine digitale Subtraktionsangiographie der Pulmonalarterien angefertigt. Anschließend führten wir einen weichen Latex-Ballonkatheter in Segment- oder Subsegmentarterien ein. Dann entfalteten wir den Ballon, um die sondierte Arterie zu verschließen und injizierten jeweils 10 bis 15 ml Kontrastmittel, um die kleinen peripheren Gefäße sichtbar zu machen.

13 Patienten litten an einer nichtthrombembolischen Form der chronischen pulmonalen Hypertonie. Bei 36 von 37 Patienten mit CTEPH fanden wir organisiertes embolisches Material als irreguläre Stenosen, Verschlüsse oder Strickleitersysteme (Webs und Bands). In der Darstellung dieser pathologischen Befunde war die Ballon-Okklusionsangiographie der Übersichtsangiographie sowohl qualitativ als auch quantitativ überlegen. Nach unseren Daten entdeckt die BallonOkklusionsangiographie in etwa bei jedem fünften Patienten mit negativer Übersichtsangiographie wenigstens ein thrombembolisches Residuum, sie steigert somit als Verfeinerung der Methode die Sensitivität der Pulmonalisangiographie. Generell stellte sie 2,7 bis 3,6 Aufteilungsgenerationen der peripheren Gefäße mehr dar als 
die konventionelle selektive DSA. Außerdem fanden wir bei 17 Patienten Kollateralgefäße zu den peripheren Segmenten von zentral verschlossenen Pulmonalarterien. Dieses Phänomen war nur in der Ballon-Okklusionsangiographie zu beobachten und erwies sich als spezifisch für Patienten mit thrombembolischer pulmonaler Hypertonie. Der Befund ist insofern erstaunlich, als dass Pulmonalarterien eigentlich als funktionelle Endarterien ohne Anastomosen zu Nachbararterien beschrieben werden. Bei 11 Patienten fanden sich Anastomosen zu subpleuralen Bronchialarterien. Dieses bereits bekannte Phänomen steht nach unseren Daten in keinem Zusammenhang mit einer bestimmten Erkrankung und ist somit als unspezifisches Merkmal der chronischen pulmonalen Hypertonie zu deuten. In drei Fällen konnten histologisch postkapilläre Formen der pulmonalen Hypertonie (zweimal pulmonale venookklusive Erkrankung (PVOD), einmal primäre kapilläre Hämangiomatose) gesichert werden. Bei diesen Patienten zeigte die Ballon-Okklusionsangiographie eine Füllung der Lungenvenen ohne angiographisch sichtbare Anfärbung des Kapillarbettes (fehlende Parenchymanfärbung). Die geschilderten Erkenntnisse aus unserer Studie lassen sich für die Praxis wie folgt zusammenfassen:

1. Die Ballon-Okklusionsangiographie verbessert die Visualisierung der peripheren Pulmonalarterien.

2. Sie erleichtert die Detektion und Lokalisation thrombembolischer Residuen

3. Sie hilft bei der Differentialdiagnose zwischen thrombembolischer und nicht-thrombembolischer chronischer pulmonaler Hypertonie.

4. Vorher unsichtbare Anastomosen und Kollateralgefäße werden sichtbar.

5. Venöse Füllung ohne Parenchymanfärbung ist offensichtlich ein Zeichen der Parenchymerkrankung; diese Konstellation ist bei Patienten mit chronischer pulmonaler Hypertonie möglicherweise ein Hinweis auf das Vorliegen der pulmonalen venookklusiven Erkrankung (PVOD) oder der primären kapillären Hämangiomatose (PCH).

6. Die selektive Ballon-Okklusionsangiographie segmentaler Pulmonalarterien verbessert in Zusammenschau mit der Computertomographie die Zuverlässigkeit in der Selektion von Kandidaten für eine pulmonale Thrombendarteriektomie oder eine Prostazyklintherapie. 


\section{Summary}

Purpose: Test the ability of selective balloon occlusion angiography of pulmonary segmental arteries in the differential diagnosis of chronic pulmonary hypertension: embolic vs. non-embolic disease, pulmonary capillary hemangiomatosis, and venoocclusive disease.

Methods and Materials: In 50 patients with pulmonary hypertension, digital subtraction angiography (DSA) of pulmonary arteries were used to assist in the selection of candidates appropriate for thromboendarterectomy. In addition to these standard methods, we introduced a soft latex balloon catheter into segmental arteries, inflated the balloon to occlude the artery, and injected 10 to $15 \mathrm{ml}$ contrast medium to visualize small peripheral vessels as completely as possible.

Results: 13 patients suffered from non-embolic pulmonary hypertension. In 36 of 37 patients with embolic pulmonary hypertension organizing embolic material was depicted as irregular narrowing or occlusion of pulmonary arteries, and weblike strictures. In all of these patients occlusion technique revealed more tiny webs or organized micro emboli in small peripheral arteries. According to our data balloon occlusion angiography discovers in every fifth patient showing a negative conventionel pulmonary angiography at least one thromboembolic residuum and thus increases as a sophisticated method the sensitivity of the pulmonary angiography. Generally, occlusion technique revealed additional 2,7 to 3,6 ramifications of peripheral vessels in comparison to conventional selective DSA. Unexpectedly, we found in 11 patients anastomoses to bronchial arteries and in 17 patients collateral vessels to the peripheral segments of centrally occluded pulmonary arteries. These findings are astonishing, because pulmonary arteries are believed ramifying dichotomically without anastomoses. Obviously, there are alterations of pulmonary perfusion, which overcome normal anatomy. 3 patients with characteristic CT signs of interstitial disease (poorly defined nodular opacities and septal lines) underwent lung biopsy: 2 cases of venoocclusive disease, 1 case of pulmonary capillary hemangiomatosis. Occlusion angiography in these 3 patients revealed filling of veins without opacification of capillaries (failing parenchymal 
phase).

Conclusion: Balloon occlusion technique improves the visualization of peripheral pulmonary arteries. Differential diagnosis of embolic and non-embolic pulmonary hypertension is facilitated. Previously invisible anastomoses and collateral vessels become visible. Venous filling without capillary opacification is apparently a sign of parenchymal disease; in patients with chronic pulmonary hypertension it might be a hint at venoocclusive disease or pulmonary capillary hemangiomatosis. 
- Abenhaim L, Moride Y, Brenot F, Rich S, Benichou J, Kurz X, Higenbottam T, Oakley C, Wouters E, Aubier M, Simonneau G, Begaud B. Appetitsuppressant drugs and the risk of primary pulmonary hypertension. N Engl J Med 1996; 335: 609-16

- Archibald CJ, Auger WR, Fedullo PF. Long-term outcome after pulmonary thromboendarterectomie. Am J Respir Crit Care Med 1999; 160: 523-8

- Barst RJ, McGoon M, Torbicki A, Sitbon O, Krowa MJ, Olschewski H, Gaine S. Diagnosis and differential assessment of pulmonary arterial hypertension. J Am CollCardiol 2004 Jun 16; 43(12): 40-47

- Bongartz G, Boos M, Scheffler K, Steinbrich W. Pulmonary circulation. Eur Radiol 1998; 8:698-706

- Bookstein JJ. Segmental arteriography in pulmonary embolism. Radiology 1969; 93: 1007-1012

- Bortz J, Lienert GA., Boehnke K. „Verteilungsfreie Methoden in der Biostatistik“. 2. Auflage 2000; Springer Berlin

- Brett W, Jamieson SW, Zerkowski HR. Pulmonale Thrombendarterektomie. Schweiz Med Forum Aug 2001; 34:855-60

- Bynum LJ, Wilson JE, Christensen EE, Sorensen C. Radiographic techniques for balloon-occlusion pulmonary angiography. Radiology 1979 Nov; 133(2): 518-20

- Duncker HR. Der Atemapparat. Benninghoff Anatomie, Urban \& Schwarzenberg; 13./14. Auflage 1985: 307-388

- Endrys J, Hayat N, Cherian G. Comparison of bronchpulmonary collaterals and collateral blood flow in patients with chronic thromboembolic and primary pulmonary hypertension. Heart 1997; 78: 171-176

- Fedullo PF, Auger WR, Kerr KM, Rubin LJ. Chronic thromboembolic pulmonary hypertension. N Engl J Med Nov 15 2001; 345(20): 1465-72

- Ferris EJ, Holder JC, Lim WN, Angtuaco EJ, Boyd CM, Binet EF, Baker JT, Bissett JK. Angiography of pulmonary emboli: digital studies and balloonocclusion cineangiography. AJR Am J Roentgenol 1984 Feb; 142(2): 369-73

- Ferris EJ, Smith PL, Lim WN, Whittle JL, Bon Bice C, Angtuaco T; Radionuclide-guided balloon occlusion pulmonary cineangiography: an adjunct to pulmonary angiography. Am Heart J 1984; 108: 539-42 
- Frazier AA, Galvin R, Franks TJ, Rosado-de-Christenson. Pulmonary Vasculature : Hypertension and Infarction. Radiographics 2000; 20: 491-524

- Geßler JR. „Statistische Graphik“. Birkhäuser 1993, Basel

- Greene R, Jantsch H, Boggis C, Strauss HW, Lowenstein E. Respiratory Distress Syndrome with New Considerations. Radiologic Clinics of North America 1983 Feb; 21(4): 699-708

- Hagspiel KD, Polak JF, Grassi CJ, Faitelson BB, Kandarpa K, Meyerovitz MF. Pulmonary embolism: comparison of cut-film and digital pulmonary angiography. Radiology Apr 1998; 207(1): 139-145

- Jacobson G. Peripheral pulmonary (wedge) arteriography. A standardized technique for the single film arteriogramm. Clin Radiol 1963; 14: 326

- Jamieson SW, Kapelanski DP. Pulmonary endarterectomie. Curr Probl Surg 2000; 37: 165-252

- Johnson MS, Stine SB, Shah H, Harris VJ, Ambrosius WT, Trerotola SO. Possible pulmonary embolus: evaluation with digital subtraction versus cutfilm angiography - prospective study in 80 patients. Radiology Apr 1998; 207(1):131-138

- Kauczor HU, Heussel CP, Thelen M. Radiodiagnostik der Lunge. Radiologe 2000; 40(10): 870-7

- Kauczor HU, Schwickert HC, Mayer E, Schweden F, Schild HH, Thelen M. Spiral CT of bronchial arteries in chronic thrombembolism. J Comput Assist Tomogr 1994; 18(6): 855-861

- Klepetko W, Mayer E, Sandoval J, Trulock EP, Vachiery JL, Dartevelle P, Jamieson SW, Lang I, Corris P. Interventional and surgical modalities of treatment for pulmonary arterial hypertension. J Am Coll Cardiol June 2004; 43: $73-80$

- Kunieda T, Nakanishi N, Satoh T, Kyotani S, Okano Y, Nagaya N. Prognoses of primary pulmonary hypertension an chronic majorvessel thrombembolic hypertension determined from cumulative survival curves. Intern Med 1999; 38: 543-6

- Ley S, Kramm T, Kauczor HU, Mayer E, Heussel CP, Thelen M, Kreitner KF. Erfassung hämodynamischer Parameter bei Patienten mit chronischer thrombembolischer pulmonaler Hypertonie mittels MRT vor und nach Thrombendarteriektomie. RöFo 2003 Dec; 175: 1647-1654

- Lorenz R: Bildgebende Verfahren. Classen M, Diehl V, Kochsiek K: Innere Medizin, Urban \& Schwarzenberg 1991; 28-48 
- Ludwig JW, Verhoeven LAJ, Kersbergen JJ, Overtoom TT. Digital subtraction angiography of the pulmonary arteries for the diagnosis of pulmonary embolism. Radiology 1983; 147: 639-645

- Matsumoto JS, Hoffman AD. Pediatric case of the day: pulmonary venoocclusive disease. AJR Am J Roentgenol 1993; 160: 1331-1332

- Mayer E, Dahm M, Hake U, Schmid FX, Pitton M, Kupferwasser I, Iversen S, Oelert H. Mid-term results of pulmonary thromboendarterectomie for chronic thromboembolic pulmonary hypertension. Ann Thorac Surg 1996; 61: 1788-92

- Moser KM, Auger WR, Fedullo PF, Jamieson SW. Chronic thromboembolic pulmonary hypertension: clinical picture and surgical treatment. Eur Respir J 1992; 5: 334-342

- Moser KM, Auger WR, Fedullo PF. Chronic major-vessel thromboembolic pulmonary hypertension. Circulation 1990; 81: 1735-43

- Moser KM, Daily PO, Peterson K, Dembitsky W, Vapnek JM, Shure D, Utley J, Archibald C. Thromboendarterectomie for chronic, major-vessel thromboembolic pulmonary hypertension: immediate and long-term results in 42 patients. Ann Intern Med 1987; 107: 946

- Nilsson T, Carlsson A, Mare K. Pulmonary angiography : a safe procedure with modern contrast media and technique. Eur Radiol 1998; 8: 86-89

- Olschewski H, Seeger W. Pulmonary hypertension - Pathophysiology, diagnosis, treatment and development of a pulmonary-selective therapy. UNI-MED 2002, Bremen

- Olschewski H., Ghofrani A., Wiedemann R., Rose F., Enke B., Gessler T., Voswinckel R., Kohstall M., Grimminger F., Seeger W. Pulmonaler Hochdruck. Internist 2002; 43: 1498-1509

- Petkov V, Doberer D. Pulmonale Hypertonie. J Hypertonie 2003; 7(3): 7-15

- Pitton MB, Kemmerich G, Herber S, Schweden F, Mayer E, Thelen M. Chronische thrombembolische pulmonale Hypertonie: Diagnostische Wertigkeit von Mehrschicht-CT und selektiver Pulmonalis-DSA. RoFo April 2002; 174(4): 474-9

- Resten A, Maitre S, Humbert M, Sitbon O, Capron F, Simoneau G, Musset D. Pulmonary Arterial Hypertension: Thin-Section CT Predictors of Epoprostenol Therapy Failure. Radiology 2002; 222: 640-644

- Rich S. Primary pulmonary hypertension: executive summary from the World Symposium on Primary Pulmonary Hypertension. Evian, France: World Health Organization, 1998 
- Robb GP, Steinberg I. Practical method of visualisation of chambers of heart, pulmonary circulation and great blood vessels in man. J. Clin. Invest. 1938; 17: 507

- Ryan KL, Fedullo PF, Davis GB. Perfusion scan findings understate the severity of angiographic and hemodynamic compromise in chronic thromboembolic pulmonary hypertension. Chest 1988; 93: 1180-85.

- Sachs L. „Angewandte Statistik“. 11. Auflage 2004; Springer Berlin

- Schoepf UJ, Costello P. CT Angiography for Diagnosis of Pulmonary Embolism: State of the Art. Radiology 2004; 230: 329-337

- Seo JB, Im JG, Goo JM, Chung MJ, Moon WK, Lee KH, Kin IO. Comparison of contrast-enhanced CT angiography and gadolinium-enhanced MR angiography in the detection of subsegmental-sized pulmonary embolism. An experimental study in a pig model. Acta Radiol Jul 2003; 44(4): 403-10

- Shackelford GD, Sacks EJ, Mullins JD, McAlister WH. Pulmonary venoocclusive disease: case report and review of the literature. AJR Am J Roentgenol 1977; 128: 643-648

- Smith TP, Ryan JM, Brodwater BK. Acute pulmonary thrombembolism: Comparison of diagnostic capabilities of conventionel film-screen and digital Angiography. Chest 2002; 122: 968-972

- Stein PD, Henry JW, Gottschalk A. Reassessment of Pulmonary Angiography for the Diagnosis of Pulmonary Embolism: Relation of Interpreter Agreement to the Order of the involved Pulmonary Arterial Branch. Radiology 1999; 210: 689-691

- Stein PD. Pulmonary embolism Baltimore, Md: Williams \& Wilkins, $1996 ; 208$

- Stein PD. Wedge arteriography for the identification of pulmonary embolism in small vessels. Am Heart J 1971; 82: 618-623

- Sumita S, Ujike Y, Namiki A, Watanabe A Satoh O. Rupture of pulmonary artery induced by balloon occlusion pulmonary angiography. Intensive Care Med 1995 Jan; 21(1): 79-81

- Trampisch HJ, Windeler J. „Medizinische Statistik“. 2. Auflage 2000; Springer Berlin

- v. Hayek H. Die menschliche Lunge. 2. Aufl. Springer, Berlin 1970

- van Beek EJR, Bakker AJ, Reekers JA. Pulmonary embolism: interobserver agreement in the interpretation of conventional angiography and DSA images in patients with nondiagnostic lung scan results. Radiology 1996; 198: $721-724$ 
- Wagenvoort CA. Pathology of pulmonary thromboembolism. Chest 1995; 107(1): 10-17

- Weiß C. „Basiswissen medizinische Statistik“. 2. Auflage 2002; Springer Berlin

- Wilson JE, Bynum LJ. An improved pulmonary angiographic technique using a balloon-tipped catheter. Am Rev Respir Dis 1976, 114(6): 1137-44

- Wilson NJ, Culham JA, Sandor GG, Taylor GP. Pulmonary wedge angiography for prediction of pulmonary vascular disease in Down Syndrome. Cathet Cariovasc Diagn 1993 Jan; 28(1): 22-33

- Zonzin P, Vizza CD, Favretto G. Chronic thromboembolic pulmonary hypertension. Ital Heart J Suppl 2003 Oct; 4(10): 814-24 
Mein besonderer Dank gilt meinem Lehrer und Doktorvater, Herrn Prof. Dr. med. W.S. Rau für die Unterstützung und Betreuung, die ständige Diskussionsbereitschaft sowie die Überlassung des interessanten Themas.

Herrn PD Dr. med. H. Olschewski aus dem Zentrum für Innere Medizin danke ich für die überaus hilfsbereite Unterstützung bei der Planung der Arbeit sowie die Bereitstellung von Krankenakten.

Herrn Dr. G. Eichner aus dem Mathematischen Institut der JLU Gießen gilt mein Dank für die ausgesprochen engagierte Unterstützung bei der statistischen Auswertung.

Daneben gilt mein Dank auch meiner Frau Manuela und den Kindern, die mir während meiner ganzen Ausbildung und der Erstellung dieser Arbeit geduldig und hilfreich zur Seite standen.

Vor allem danke ich meinen Eltern und meiner Familie. Meine Schwestern Katja und Christiane haben durch ihr kritisches Korrekturlesen in letzter Sekunde nicht unwesentlich zum Gelingen dieser Arbeit beigetragen. 
8.

Persönliche Daten

\section{Lebenslauf}

Andreas Walter Josef Breithecker

Geboren am 17.03.1968 in Hadamar

Verheiratet mit Manuela Breithecker

Drei Töchter (Sophie (7), Emilie (5), Aenne (3))

Schulbildung

1974-1978 Grundschule in Waldbrunn/ Ellar

1978-1987 Gymnasium in Hadamar, Abitur im Mai 1987

Wehrdienst

Juli 1987- September 1988 Grundwehrdienst

Studium

Oktober 1988- Mai 1995 Studium der Humanmedizin an

der JLU Gießen, Staatsexamen im Mai 1995

Mai 1995- Januar 1996 Promotionsstudent am Institut für

Anatomie und Zellbiologie der JLU Gießen

Beruflicher Werdegang

Februar 1996- Juli 1997 AiP im Zentrum für Radiologie

sowie im Zentrum für Innere Medizin der Universitäts-

klinik Gießen

August 1997- Januar 1998 Assistenzarzt im Zentrum für

Innere Medizin der Uniklinik Gießen

Seit Februar 1998 Assistenzarzt im Zentrum für

Diagnostische Radiologie der Uniklinik Gießen

Nebentätigkeiten während des Studiums
Beschäftigungen als studentische Hilfskraft:

WS 1990/91 Physiologisches Institut

SS 1991 u. WS 1991/92 Institut für Anatomie

SS 1992

Geschichte der Medizin und

Anatomie

WS 1992/93

Anatomie

SS 1993- WS 1994/95 Geschichte der Medizin 


\section{Erklärung}

„Ich erkläre: Ich habe die vorgelegte Dissertation selbständig, ohne unerlaubte fremde Hilfe und nur mit den Hilfen angefertigt, die ich in der Dissertation angegeben habe. Alle Textstellen, die wörtlich oder sinngemäß aus veröffentlichten oder nicht veröffentlichten Schriften entnommen sind, und alle Angaben, die auf mündlichen Auskünften beruhen, sind als solche kenntlich gemacht. Bei den von mir durchgeführten und in der Dissertation erwähnten Untersuchungen habe ich die Grundsätze guter wissenschaftlicher Praxis, wie sie in der „Satzung der Justus-Liebig-Universität Gießen zur Sicherung guter wissenschaftlicher Praxis“ niedergelegt sind, eingehalten." 